University of Louisville

ThinkIR: The University of Louisville's Institutional Repository

Electronic Theses and Dissertations

$8-2020$

\title{
Model-based control methods to improve the power qualify of grid-connected single-phase inverters.
}

\author{
Moath Alqatamin \\ University of Louisville
}

Follow this and additional works at: https://ir.library.louisville.edu/etd

Part of the Controls and Control Theory Commons, and the Power and Energy Commons

\section{Recommended Citation}

Alqatamin, Moath, "Model-based control methods to improve the power qualify of grid-connected singlephase inverters." (2020). Electronic Theses and Dissertations. Paper 3493.

https://doi.org/10.18297/etd/3493

This Doctoral Dissertation is brought to you for free and open access by ThinkIR: The University of Louisville's Institutional Repository. It has been accepted for inclusion in Electronic Theses and Dissertations by an authorized administrator of ThinkIR: The University of Louisville's Institutional Repository. This title appears here courtesy of the author, who has retained all other copyrights. For more information, please contact thinkir@louisville.edu. 


\title{
MODEL-BASED CONTROL METHODS TO IMPROVE THE POWER QUALITY OF GRID-CONNECTED SINGLE-PHASE INVERTERS
}

\author{
By \\ Moath Alqatamin \\ B.S., Mu'tah University, Jordan, 2003 \\ M.S., Jordan University of Science and Technology, Jordan, 2009
}

\begin{abstract}
A Dissertation
Submitted to the Faculty of the

J.B. Speed School of Engineering of the University of Louisville

in Partial Fulfillment of the Requirements

for the Degree of

Doctor of Philosophy

in Electrical Engineering

Department of Electrical and Computer Engineering

University of Louisville

Louisville, Kentucky
\end{abstract}

August 2020 



\title{
MODEL-BASED CONTROL METHODS TO IMPROVE THE POWER QUALITY OF GRID-CONNECTED SINGLE-PHASE INVERTERS
}

\author{
By \\ Moath Alqatamin \\ B.S., Mu'tah University, Jordan, 2003 \\ M.S., Jordan University of Science and Technology, Jordan, 2009
}

A Dissertation Approved On

June 23, 2020

by the following Dissertation Committee:

Michael L. McIntyre (Dissertation Director)

Christopher Richards

Nicholas Jewell

Tamer Inanc 


\title{
ABSTRACT \\ MODEL-BASED CONTROL METHODS TO IMPROVE THE POWER QUALITY OF GRID-CONNECTED SINGLE-PHASE INVERTERS
}

\author{
Moath Alqatamin
}

June 23, 2020

Power electronic converters are commonly used for interfacing distributing generation sources (DGs) to the electrical power system networks. This is necessary because these DGs usually have different output characteristics and cannot be connected directly to the local load and/or the grid. The power electronic front-end converter is an inverter whose dc link is fed by an ac/dc converter or by a dc/dc converter, according to the DG source type. The commercial front-end inverters are designed to operate either in grid-connected (GC) mode or in stand-alone (SA) mode. In the SA mode, the inverter is connected to local load, but in the GC mode the inverter must be connected to the utility grid and a local load could be connected to this system as well. Based on this, any designed or proposed controller for such systems should work well in both operation modes. The control objective in SA mode is to improve the quality of the local load voltage, and the control objective in GC mode is to inject clean current to the grid with low total harmonic distortion (THD). Most of the control schemes in the literature have been designed to work in one of these operation modes and ensure low THD either for the local load voltage or for the injected grid current. However, some of the existing control schemes in the literature proposed different control architectures for each operation mode. Moreover, there are a few researches have been reported in the literature based on the cascaded control theory to 
obtain low THD for both the local load voltage simultaneously with the injected current to the grid in the grid-connected mode.

Due to the growing penetration of the DG sources in the residential applications, single-phase grid-connected inverters have gained much attention. For this reason, the single-phase grid-connected inverter systems have been chosen in our study. Since such systems have nonlinearity in its behavior, different nonlinear model-based control schemes have been designed in order to improve the quality of the local load voltage while injecting clean current to the grid for single-phase grid-connected inverter systems by using single structure control scheme. Furthermore, the proposed control schemes ensure the seamless transfer between GC and SA operation modes without adjusting the controller structure and with self-synchronization ability. 


\section{TABLE OF CONTENTS}

LIST OF TABLES

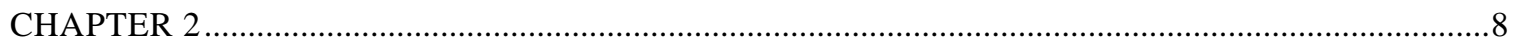

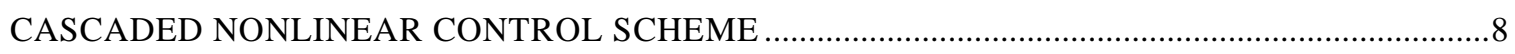

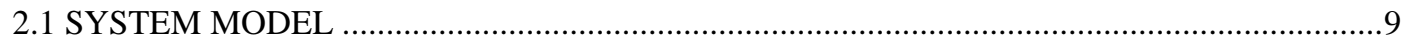

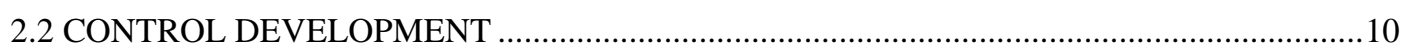

2.2.1 INNER-LOOP VOLTAGE CONTROLLER DESIGN ……..............................................11

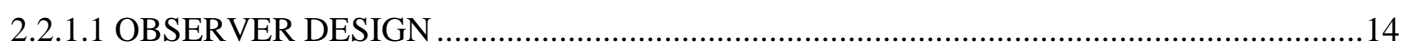

2.2.1.2 STABILITY ANALYSIS FOR THE INNER-VOLTAGE LOOP ......................................15

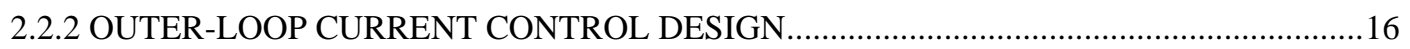

2.2.2.1 STABILITY ANALYSIS FOR THE OUTER-LOOP ........................................................19

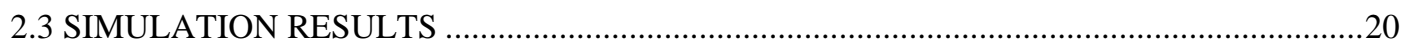

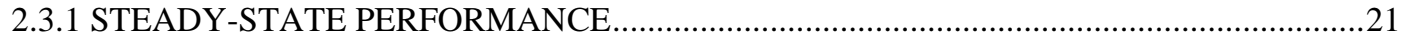

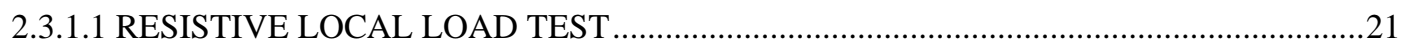

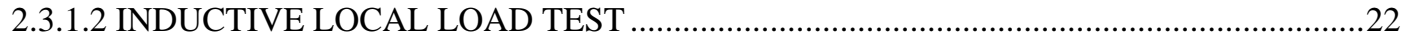

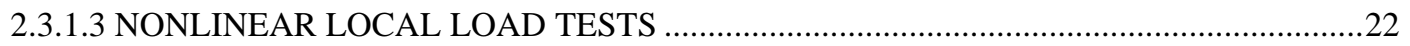




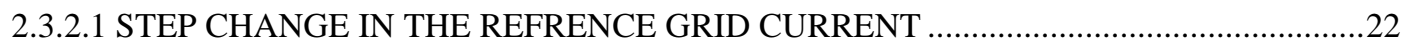

2.3.2.2 STEP CHANGE IN THE RESISTIVE LOCAL LOAD ............................................23

2.3.3 SEAMLESS TRANSITION BETWEEN GA AND SA MODES ..................................23

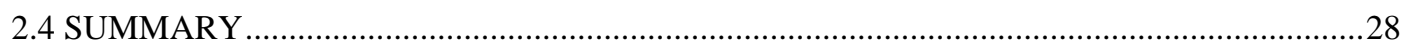

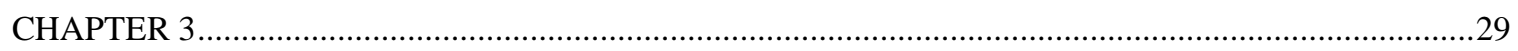

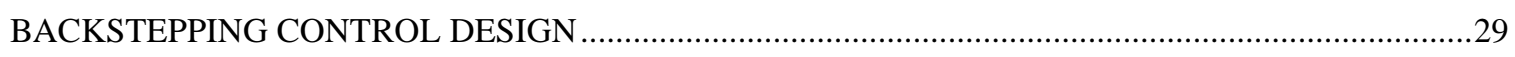

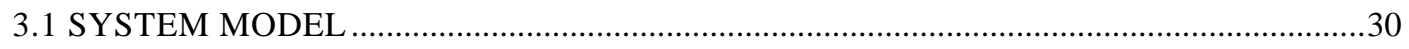

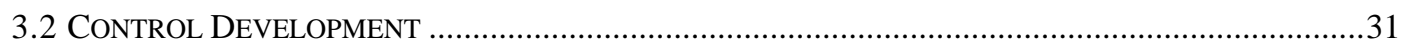

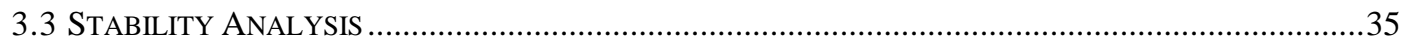

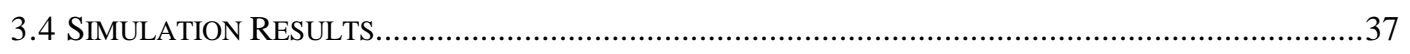

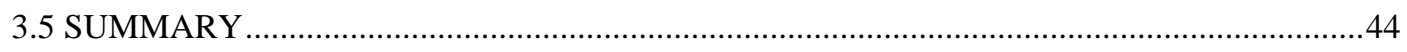

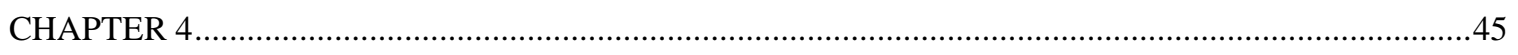

BACKSTEPPING CONTROL SCHEME WITH VARIBLE STRUCTURE OBSERVER ......................45

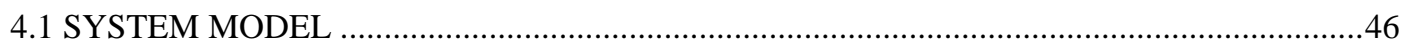

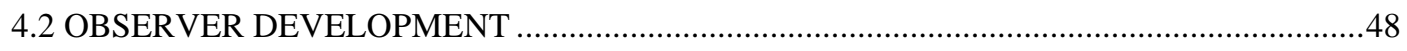

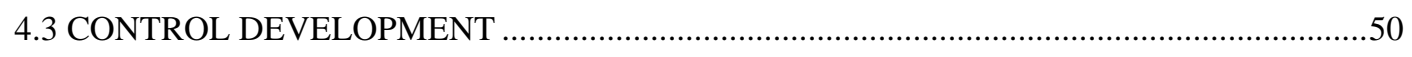

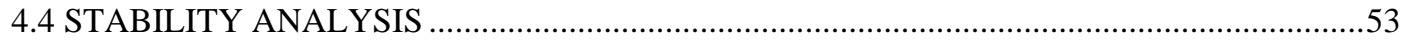

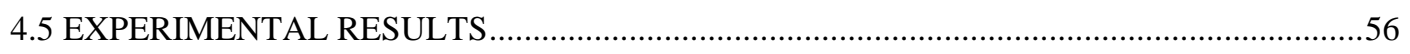

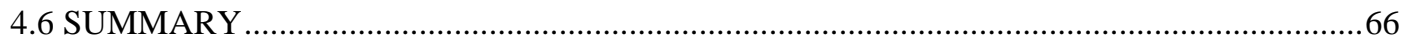

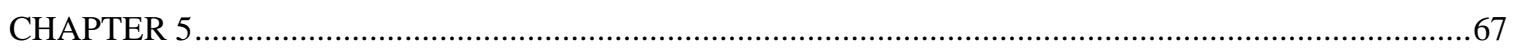

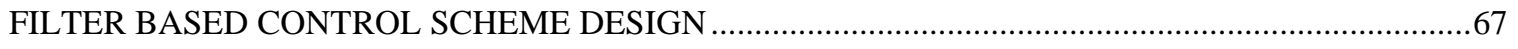

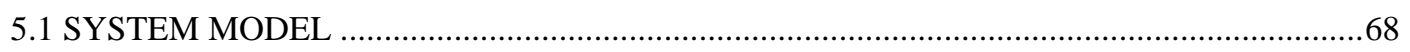




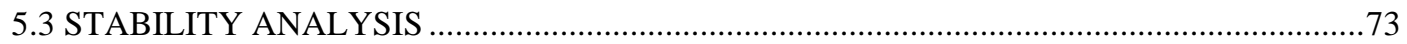

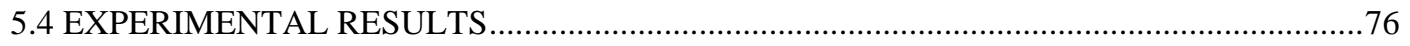

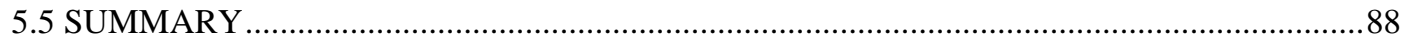

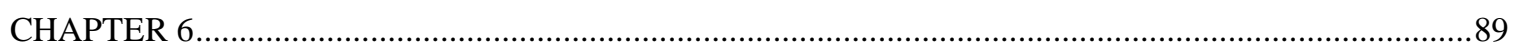

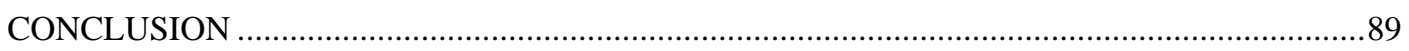

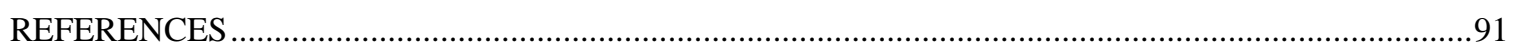

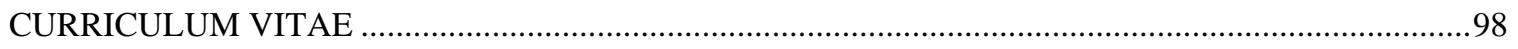




\section{LIST OF TABLES}

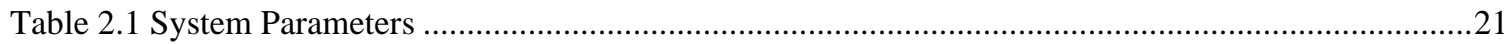

Table 2.2 Steady State Errors for cascaded controller with different types of loads ...................................25

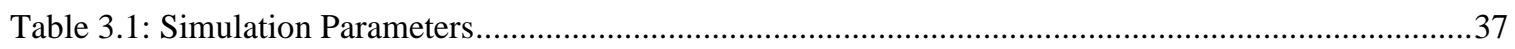

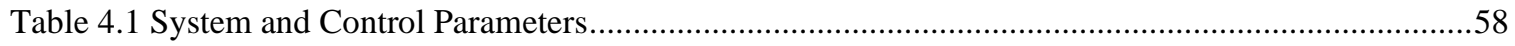

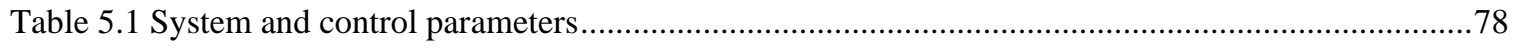

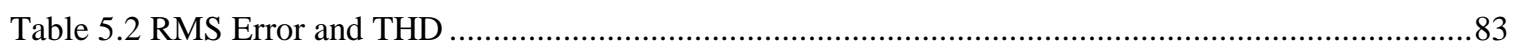




\section{LIST OF FIGURES}

Figure 2.1 Grid-connected single-phase inverter diagram........................................................................

Figure 2.2 Inverter side system for inner-loop voltage controller ............................................................11

Figure 2.3 Grid side system for outer-loop current controller ...................................................................17

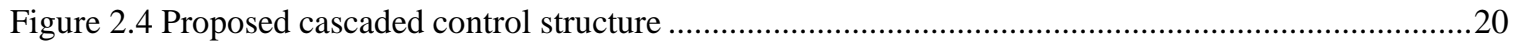

Figure 2.5 Tracking response of the output voltage for resistive local load...............................................23

Figure 2.6 Tracking response of the grid current under resistive local load................................................24

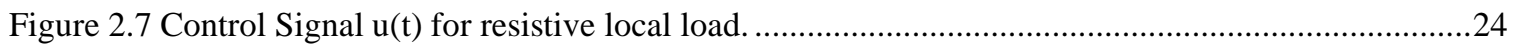

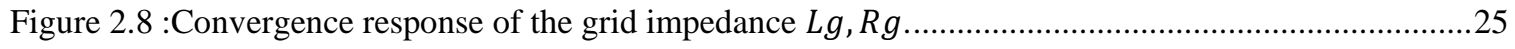

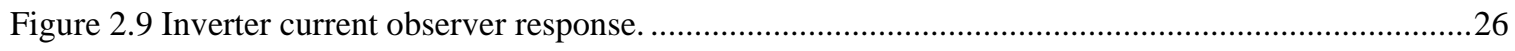

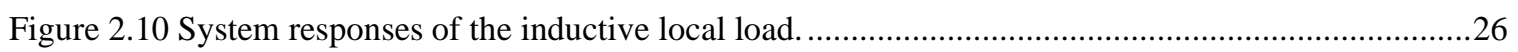

Figure 2.11 System responses of the nonlinear local load..........................................................................26

Figure 2.12 Tracking response of the grid current for step change in the reference current. .......................27

Figure 2.13 Tracking response of the grid current under load changing. ..................................................27

Figure 2.14 Current tracking response during seamless transfer between SA and GC modes....................27

Figure 2.15 Output voltage response during seamless transfer between SA and GC modes. ......................28

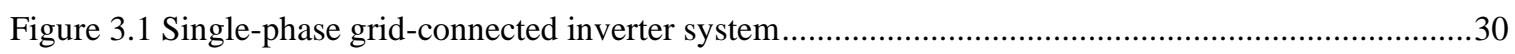

Figure 3.2 Tracking response of the output voltage for RL local load. .......................................................39

Figure 3.3 Tracking response of the grid current while the inverter supplies RL local load .........................39

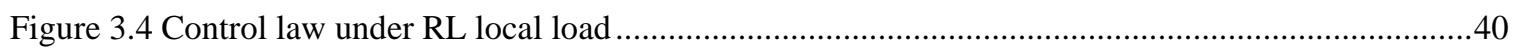

Figure 3.5 Tracking Performance of the filter inductor current under RL local load..................................40

Figure 3.6 Estimation of the output current for RL local load. .................................................................. 41

Figure 3.7 Tracking response of the output voltage for nonlinear local load. ..............................................41

Figure 3.8 Tracking response of the grid current while the inverter supplies nonlinear local load...............42

Figure 3.9 System responses of the nonlinear local load show unity power factor at grid side. …...............42

Figure 3.10 Tracking response of the grid current for $-50 \%$ step change in the reference current................43

Figure 3.11 Current tracking response during seamless transfer between SA and GC modes. .....................43

Figure 3.12 Output voltage response during seamless transfer between SA and GC modes. ......................44 
Figure 4.1 Single-phase grid-connected inverter system.

Figure 4.2 Experimental setup of the grid-connected inverter

Figure 4.3 Block diagram for the proposed controller/observer scheme .57

Figure 4.4 Block diagram for cascaded PR control scheme ......................................................58

Figure 4.5 Tracking response of the output voltage for RL local load. ..................................................60

Figure 4.6 Tracking response of the grid current under RL local load..............................................61

Figure 4.7 Observer tracking performance under RL local load. ......................................................61

Figure 4.8 Control law for the proposed scheme under RL local load. ...............................................62

Figure 4.9 Tracking response of the output voltage for NL local load.............................................62

Figure 4.10 Tracking response of the grid current under NL local load............................................63

Figure 4.11 System response to show unity power factor under NL local load. .....................................63

Figure 4.12 Tracking response of the grid current during reference current step change under NL load (a)

Proposed Scheme (b) Cascaded PR controller (required gains tuning) .............................................64

Figure 4.13 Voltage and current tracking response during transfer from SA mode to GC mode. (a) Proposed

Scheme (b) Cascaded PR controller (required gains tuning)...... .65

Figure 4.14 Voltage and Current tracking response during transfer from GC mode to SA mode (a) Proposed

Scheme (b) Cascaded PR controller (required gains tuning) .............................................................65

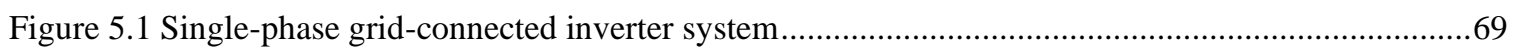

Figure 5.2 Experimental setup of the grid-connected inverter .................................................

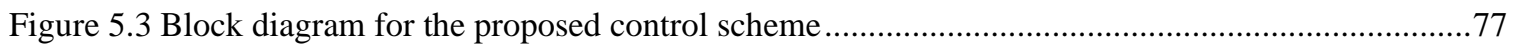

Figure 5.4 Tracking response of the output voltage for RL local load (a) Proposed Scheme, (b) Cascaded PR scheme .81

Figure 5.5 Tracking response of the grid current under RL local load (a) Proposed Scheme, (b) Cascaded PR scheme....... .82

Figure 5.6 Control law for the proposed scheme under RL local load 82

Figure 5.7 Tracking response of the output voltage for NL local load. (a) Proposed Scheme, (b) Cascaded PR scheme (required gains tuning). 
Figure 5.8 Tracking response of the grid current under NL local load. (a) Proposed Scheme, (b) Cascaded

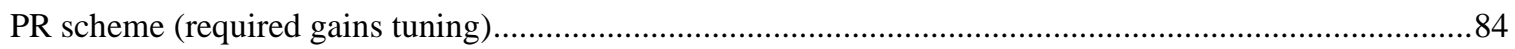

Figure 5.9 System response to show unity power factor under NL local load. ...........................................84

Figure 5.10 Tracking response of the grid current during reference current step change under NL load (a)

Proposed Scheme, (b) Cascaded PR scheme (required gains tuning). ........................................................85

Figure 5.11 Voltage and current tracking response during transfer from SA mode to GC mode (a) Proposed

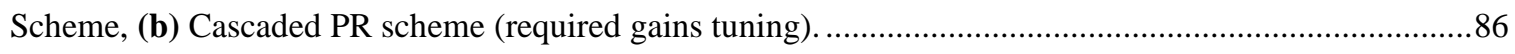

Figure 5.12 Voltage and Current tracking response during transfer from GC mode to SA mode (a) Proposed

Scheme, (b) Cascaded PR scheme (required gains tuning). ........................................................................ 86

Figure 5.13 Hardware-in-the-loop (HIL) schematic diagram with LabVIEW cRIO.....................................87

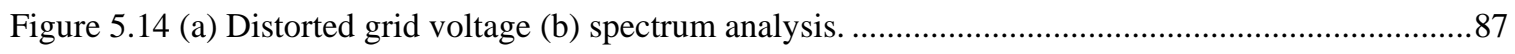

Figure 5.15 Tracking response of the grid current under distorted grid condition for NL local load............88 


\section{CHAPTER 1}

\section{INTRODUCTION}

Much interest has been raised in recent times in the micro-grid (MG) systems arena. Renewable energy sources, such as wind turbines, solar cells, and micro turbines are used as primary sources to MG systems. Inverters have been widely used as dc/ac power converter devices in MG systems to interface renewable energy sources to the utility grid and/or local load because the renewable energy sources have different output characteristics [1]. The MG system can be connected to the grid in the grid-connected mode (GC) or can be worked as an islanded unit in the stand-alone mode (SA) [2]. In the GC mode the inverters in the MGs behave as current source and it is usually connected to the grid through LCL filter or LC filter with grid interface inductance in order to attenuate the switching frequency harmonics [3]. Typically, in the GC mode; the injected current to the grid should have low total harmonic distortion (THD) and within the IEEE standard acceptable limits for such systems [4]. In smart inverter systems, Controlling the phase shift between the injected current to the grid and the phase angle of the grid can determines which type of the power could be delivered to the grid, active or reactive power [5-8]. In order to obtain unity power factor at the grid side this current should be in phase with the grid. Unity power factor at the grid side means only active power has been injected to the grid. Most commercial single-phase grid-connected inverters have been controlled to inject only active power and ensure unity power factor at the grid side [9]. On the other hand, in SA mode the inverters work as a voltage source which should supply the local load with a low THD voltage of the appropriate magnitude, regardless of the nature of the load [10], [11]. 
From the microgrid control view, improving the performance of grid-connected inverters is the main objective of many control approaches in literature. The performance of such systems can be measured by the quality of the local load voltage and the quality of the injected current to the grid. In [12], current control of a single-phase grid-connected inverter with transformer isolation has been achieved via a digital model predictive controller. However, this scheme does not consider the dynamic of the grid or the local load voltage. Similarly, a controller based on Control Lyapunov Function (CLF) has been proposed in [13] to control the injected current to the grid. Moreover, a current controller based on composite nonlinear feedback (CNF) is proposed in [14] for a grid-connected inverter. The proposed CNF controller combines linear and nonlinear feedback signals to improve the closed-loop transient and steady-state response of the system. In [15], the proposed current control scheme has been designed based on Sliding Mode Control (SMC) to inject clean current to the grid. In general, the chattering problem is common in SMC theory. In addition, taking the numerical derivative of the capacitor voltage is another problem in [15], as this causes amplification of the measured noise. Moreover, the authors in [16-19] also present enhanced current controllers in order to improve the performance of grid connected inverters operating in weak and distorted grid. Recently, The backstepping technique [20] has been utilized to design a nonlinear control for both stand-alone and grid-connected inverter systems. For instance, A backstepping controller based on high order sliding mode for three phase grid-connected inverter has been designed in [21] to regulate the grid current. A current controller based on $H^{\infty}$ and repetitive control techniques has been proposed in [22] to inject clean current to the grid in three-phase grid-connected inverter. In contrast, the same approach has been used in [23] to a design voltage controller for grid-connected 
inverter to keep low THD for the local load voltage. Moreover, a backstepping nonlinear controller has been proposed in [24] to regulate the output voltage and frequency for a threephase grid-connected inverter without studying the quality of the grid current. Also, the authors in [25] designed a nonlinear controller based on backstepping methods to enhance the quality of the output voltage in the presence of a nonlinear local load.

For microgrid applications, the inverter systems should have the ability to work in two operation modes; SA and GC modes. As a result, the controllers for such systems are required to operate in two modes as well. For this purpose, the authors in $[26,27]$ have designed a distinct controller for each mode. The control scheme eliminates the impact of the nonlinear local load on the grid current in the grid-connected mode. When the microgrid detects the islanding situation, a voltage control mode should be inserted to assure acceptable quality of the local load output voltage waveform. Similarly, the operation of the inverters in $[28,29]$ have been divided into two modes, grid-connected mode and standalone mode. Each mode has different control architecture. Moreover, Two nonlinear controllers based on backstepping approach have been designed in [30]. One of them to control the local load voltage in stand-alone mode, whereas the other is proposed to control the output current in grid-connected mode. Once the islanding status is detected, the transition scheme is activated to change between these two controllers.

From the previous discussion, it can be noted that there are different solutions in literature for obtaining low THD and controlling either the output voltage in sand-alone mode or the grid current in grid-connected mode by different controller schemes for each mode. However, the challenge is to meet the two objectives in grid-connected mode simultaneously by using one controller. In general, the cascaded control theory is typically 
utilized to achieve two or more control objectives [31-35]. A few of those works have been proposed to simultanously control the output local load voltage and the injected current to the grid for grid-connected inverter systems. For instance, the proposed cascaded currentvoltage controller in [34] has been designed based on $H^{\infty}$ repetitive control strategy. In [34], the current controller is in the outer loop, whereas the voltage controller is in th inner loop.The designed controller is applied to improve the power quality of the local load voltage and the grid current at the same time for the three-phase grid-connected inverter. Moreover, the authors in [35] have proposed a fixed hysteresis control scheme based on the SMC technique. The controller includes an inner voltage loop with linear proportional (P) controller and an outer current loop with linear proportional-integral (PI) controller. In [36], the authors proposed a multiloop control scheme to inject clean current to the grid. They use the inner loop voltage control as an active damping technique for LCL resonance and the outer loop current control to control the grid current. For the same purpose, the authors in [37] proposed a control scheme by using boundary control method and deadbeat controller. The boundary control is employed in the inner loop by feeding back the capacitor current and voltage to reduce the system order seen from the deadbeat controller in the outer loop.

In this dissertation, a single-phase inverter has been connected to the grid through LC filter and grid interface inductance. The inverter will be sized to supply current to a parallel connected local load and to the grid, simultaneously. In such systems, there are many sources of time varying uncertainties and nonlinearities such as local load current, grid voltage and switching dynamics of the inverter. In this work, we propose different modelbased nonlinear control schemes to improve the power quality of the local load voltage and grid current of the single-phase grid-connected inverter systems simultaneously by using 
one control structure. Furthermore, the proposed control schemes ensure the seamless transfer between grid-connected and stand-alone operation modes without adjusting the controller structure and with self-synchronization ability.

Firstly, To start from the common approach in the literature, cascade control theory has been employed along with the nonlinear control theory to design a model-based nonlinear cascaded controller to improve the quality of the local load voltage and to inject clean current to the grid, simultaneously [38]. The proposed control scheme has an outer current loop and an inner voltage loop. Power quality of the local load voltage is the responsibility of the inner voltage controller. The role of the outer current controller is to inject clean current to the grid. In the previous works [34] and [35] the authors have used two current sensors to measure the inverter filter current and the grid current in the grid interface inductance. In our work [38], a current observer has been designed to replace the inverter filter current sensor in order to reduce the impact of switching noise present in this measurement, along with system cost. In general, the main disadvantage of cascaded control schemes is that they require the objective in the inner control loop to be met before activating the outer control loop. To ensure this, a supervisory control is needed in the cascaded approach and the controller in the outer loop should be designed to have a slower dynamic response than the controller in the inner loop. Cascaded control further assumes that the stability of the two control loops are independent. As an effort to overcome these shortfalls of the cascaded control approaches, the backstepping theory [20] has been utilized to design a nonlinear controller to achieve the previous two control objectives simultaneously [39]. The proposed control scheme in [39] came at the requirement of full state-feedback which is both costly and introduces the noisy inverter inductor current signal 
to the control scheme. This approach also required real-time numerical differentiation of the grid voltage measurement which is also problematic. To overcome the aforementioned disadvantages of proposed backstepping control approach in [39], a novel second order dynamic system equation in terms of the output voltage instead of coupled inductorcapacitor dynamic system model from [35] has been utilized to design a backstepping control scheme. As a result of the novel modeling, the derivative of the output voltage has been introduced in this dynamic systems equation. To deal with this term, a variable structure nonlinear observer was developed [40].

As it is seen from previous discussion, in the proposed schemes in [39, 40], the numerical and mathematical derivative of the grid voltage are required to be known. Moreover, the approach in [40] requires to design variable structure observer to avoid the numerical derivative of the output voltage which increases the system complexity. As an effort to overcome the above disadvantages of above approaches, a nonlinear filter-based control approach [41] has been proposed. In this scheme the second order dynamic system equation in term of the output voltage has been utilized to design the proposed controller. As a result, the need for a current sensor is removed, which will reduce the system costs and as such, the measurement is not polluted with switching noise. Thus, creating a control scheme relies only on the output measurements. Moreover, in this proposed filter-based control scheme, the derivative of the output voltage and the grid voltage has been avoided.

For each developed control scheme in this work, the seamless transition between stand-alone mode and grid-connected mode is ensured without changing the controller structure. Moreover, a Lyapunov stability analysis is presented which proves that the voltage and current tracking objectives are achieved by the same controller with all signals 
remaining bounded. An experimental test bed has been implemented to further validate the proposed control schemes in the real-time environment as well the performance is compared to a conventional approach. In this hardware setup, a programmable DC power supply BK-PRECISION XLN30052 1.56KW is used as input DC voltage of the H-bridge single-phase inverter. A two-quadrant programmable AC source BK- PRECISION 9803 750VA was used to emulate the utility grid at fundamental frequency $60 \mathrm{~Hz}$. The NI CompactRIO 9063 with LabVIEW software has been used to implement the proposed algorithm and to execute it in real-time by the onboard Virtex-5 LX50 FPGA.

The remainder of this work will proceed as follows: in Chapter 2, a cascaded nonlinear controller has been proposed to control the local load voltage and the grid current simultaneously. A learning approach has been utilized in the voltage loop to avoid the need for a numerical derivative of the reference trajectory of the output voltage, which is generated from the outer current loop. In chapter 3, the backstepping control approach has been designed based on the standard third order dynamic equations of the grid-connected inverter system. To reduce the number of the current sensors in the proposed backstepping approach from chapter 3, a novel second order dynamic system equation in term of the output voltage has been utilized in chapter 4 instead of the standard coupled inductorcapacitor dynamics. As a result, the need for a filter inductor current sensor is removed, which will reduce the system costs and as such, the measurement is not polluted with switching noise. To overcome the disadvantages of the proposed control schemes in chapters 3 and 4, the novel filter-based control scheme has been designed in chapter 5 . Finally, conclusions are discussed in chapter 6. 


\section{CHAPTER 2}

\section{CASCADED NONLINEAR CONTROL SCHEME}

In this chapter, a cascaded control scheme based on nonlinear methods has been designed to simultaneously improve the quality of the local load voltage while also controlling the injected grid current in a grid-connected single-phase inverter system. This control approach ensures the seamless transfer between grid-connected and stand-alone operation modes without adjusting the controller structure. The proposed control structure consists of an outer current loop and inner voltage loop, each of which are motivated by separate Lyapunov based stability analysis. In an effort to reduce cost and noise sensitivity an inductor current observer is utilized. This scheme incorporates a Learning scheme to compensate for periodic disturbances which are present in the dynamic system. This scheme has been used in [42] to avoid the derivative of the output current in a stand-alone voltage inverter. Moreover, since the impedance of the grid has significant effect on system stability and current control performance, parameter estimation scheme is developed to compensate for this unknown parameter [43]. Each scheme in the cascaded system is validated through a Lyapunov stability analysis. The overall scheme is validated with an instantaneous circuit simulation where PLECS software was utilized.

The remainder of this chapter is organized as follows. The mathematical model of the single-phase grid-connected inverter with LC filter is presented in Section 2.1. In Section 2.2, this model is then used to develop the cascaded controller and current observer. A Lyapunov stability analysis is performed for each control loop in this section. The simulation results in Section 2.3 validate the controller development. Concluding remarks are provided in Section 2.4 . 


\subsection{SYSTEM MODEL}

A single-phase grid-connected inverter with LC filter is shown in Fig.1. The system consists of the following elements: DC power supply $V_{d c}$, H-bridge voltage source inverter (VSI), LC filter $L_{f}, C_{f}$, where $R_{f}, R_{c}$ are the series resistances of the inductor and capacitor,

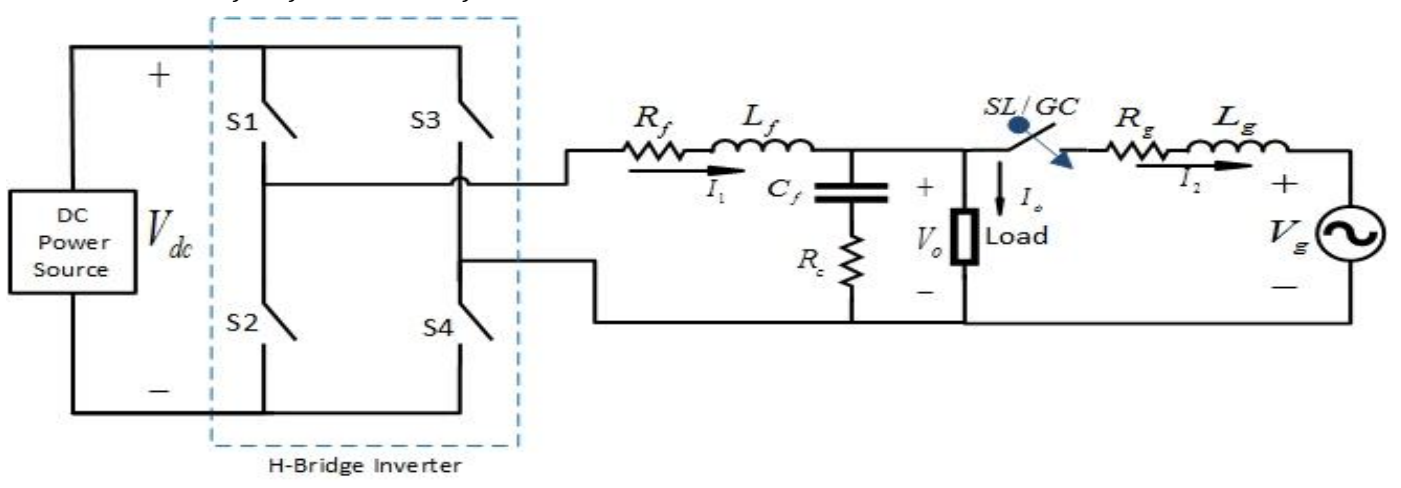

Figure 0.1 Grid-connected single-phase inverter diagram

respectively. The grid is represented by an ac voltage source $V_{g}$ and impedance which is considered as an inductance $L_{g}$ with an internal series resistance $R_{g}$. A local load has been connected in parallel to the capacitor $C_{f}$. By applying KVL and KCL to the state average model of the circuit, the mathematical differential equations representing the dynamic system are:

$$
\begin{gathered}
L_{f} \dot{I}_{1}=-\left(R_{f}+R_{c}\right) I_{1}-V_{c}+R_{c}\left(I_{2}+I_{o}\right)+u V_{d c} \\
C_{f} \dot{V}_{c}=I_{1}-I_{2}-I_{o} \\
L_{g} \dot{I}_{2}=-R_{g} I_{2}+V_{o}-V_{g}
\end{gathered}
$$

where $I_{1}(t), V_{c}(t), I_{2}(t) \in \mathbb{R}$ are the inductor output current, capacitor voltage, and injected current to the grid, respectively. $V_{o}, I_{o} \in \mathbb{R}$ are the voltage and current of the local load, respectively. $u(t) \in \mathbb{R}$ is the subsequently design control signal. 


\subsection{CONTROL DEVELOPMENT}

To facilitate the control development, the system is divided into two subsystems. The first subsystem (grid side) is the grid utility with its grid impedance. The second system (inverter side) consists of the DC supply, VSI, LC-filter, and the local load. Based on the theory of the cascaded control, and in recognition of the fact that the dynamic of the outer loop (grid frequency) is much slower than the inner loop (switching frequency), we are able to justify designing the two control loops separately [34]. Accordingly, the inverter side system will be used to design the inner-loop voltage controller and the grid side system will be used to design the outer-loop current controller with the assumption that the control objective of the inner-loop has been met. The subsequent development is based upon the following assumptions:

Assumption 1: $R_{f}, L_{f}, C_{f}, R_{c}, V_{d c}$ are known, constant system parameters.

Assumption 2: The output voltage $V_{o}(t)$ is measurable and the grid voltage $V_{g}(t)$ is measurable and bounded.

Assumption 3: The current of the grid side system $I_{2}(t)$ can be considered as a slowly time-varying external disturbance, hence $d \triangleq I_{2}$. It is also practical to assume that $\dot{d} \approx 0$. The dynamics of the grid current will be orders of magnitude slower than the switching dynamics of the inner loop control scheme [44]. 


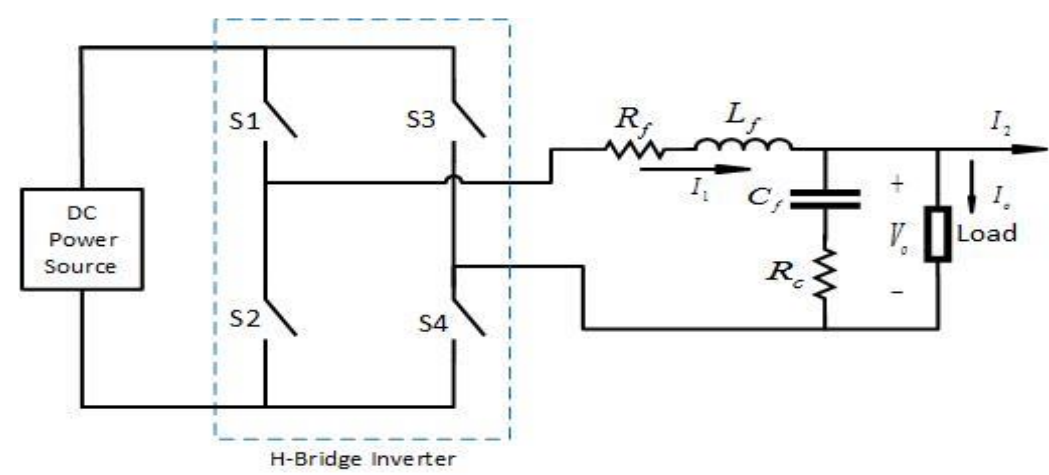

Figure 0.2 Inverter side system for inner-loop voltage controller

Assumption 4: $R_{g}, L_{g}$ are unknown constant grid impedance parameters in the sense $\dot{R}_{g}, \dot{L}_{g} \approx 0$.

Assumption 5: The reference current trajectory and its derivative are known and bounded, $I_{2 r e f}(t), \dot{I}_{2 r e f}(t) \in \mathcal{L}_{\infty}$.

Assumption 6: The local load current and its first derivative are bounded, $I_{o}, \dot{I}_{o} \in \mathcal{L}_{\infty}$. Assumption 7: the inductor current is bounded $I_{1}(t) \in \mathcal{L}_{\infty}$, for bounded values of $V_{o}(t)$.

\subsubsection{INNER-LOOP VOLTAGE CONTROLLER DESIGN}

Fig.2 shows the inverter side system that will be used to design the voltage controller in the inner loop. The control objective of the inner-loop voltage controller is to maintain low THD of the local load voltage regardless of the type of load and achieve the reference output voltage trajectory $V_{o_{\text {ref }}}(t)$, hence $V_{o}(t) \rightarrow V_{o_{\text {ref }}}(t)$ as $t \rightarrow \infty$. An additional assumption is required such that a stable inner closed loop control scheme is possible. As such we assume the outer current loop will only provide a bounded reference signal for the inner loop, as such we assume the following: 
Assumption 8: The reference voltage trajectory is bounded, $V_{o_{r e f}}(t) \in \mathcal{L}_{\infty}$ as shown in Fig.2.4. This is typically done with the cascaded approaches implementation, and as such a supervisory system is in place to stop the system operation if this assumption is not meet.

From Fig.2, and the Assumption 3, the local load voltage $V_{o}(t)$ can be written as:

$$
V_{o}=V_{c}+R_{c}\left(I_{1}-d-I_{o}\right)
$$

After taking the time derivative of (4), and using (2), the result is (5):

$$
\dot{V}_{o}=\frac{I_{1}}{C_{f}}-\frac{d}{C_{f}}-\frac{I_{o}}{C_{f}}-R_{c} \dot{I}_{o}+R_{c} \dot{I}_{1} .
$$

Substituting $V_{c}$ from (4) into (1), and using Assumption 3 yields:

$$
L_{f} \dot{I}_{1}=-R_{f} I_{1}-V_{o}+u V_{d c}
$$

Substituting (6) in (5) after multiplying (5) by $L_{f}$ along with some mathematical simplifications, the dynamic of the output voltage will be as:

$$
L_{f} \dot{V}_{o}=-A I_{1}-B d-R_{c} V_{o}-B I_{o}-R_{c} L_{f} \dot{I}_{o}+R_{c} u V_{d c}
$$

where $A \triangleq R_{f} R_{c}-\frac{L_{f}}{C_{f}}$, and $B \triangleq \frac{L_{f}}{C_{f}}$.

To facilitate the design of the control signal $u(t)$, the tracking error signal $e(t) \in \mathbb{R}$ is defined as:

$$
e \triangleq V_{\text {ofef }}-V_{o}
$$

Pre-multiplying (8) by $L_{f}$, taking the time derivative and substituting (7), the following open loop error dynamic for $e(t)$ is obtained:

$$
L_{f} \dot{e}=\left[L_{f} \dot{V}_{o r e f}+B I_{o}+R_{c} L_{f} \dot{I}_{o}\right]+A I_{1}+B d+R_{c} V_{o}-R_{c} u V_{d c}
$$


Since the reference output voltage trajectory $V_{o_{r e f}}(t)$ is generated from the outer-loop current controller, it is not a practical solution to use the numerical derivative of this signal $\dot{V}_{\text {ref }}$ in our control law. To solve this problem, a learning approach will be used in this paper to compensate for the following lumped non-state depending periodic disturbance [45]:

$$
d_{1} \triangleq L_{f} \dot{V}_{\text {ref }}+D I_{o}+R_{c} L_{f} \dot{I}_{o}
$$

The open loop error equation after inserting (10) into (9) is as follows:

$$
L_{f} \dot{e}=d_{1}+A I_{1}+B d+R_{c} V_{o}-R_{c} u V_{d c}
$$

Motivated by the subsequent stability analysis, the control law $u(t)$ is defined as:

$$
u \triangleq \frac{1}{R_{c} V_{d c}}\left[\hat{d}_{1}+A \hat{I}_{1}+B \hat{d}+R_{c} V_{o}+K e\right]
$$

where $K \in \mathbb{R}^{+}$is a control gain, $\hat{d}(t), \hat{d}_{1}(t)$ are the estimation of the system disturbances which will be designed later, and $\hat{I}_{1} \in \mathbb{R}$ is the inductor current observer which will be designed in the next section. Substituting (12) in (11), the closed loop error dynamic for $e(t)$ is defined as:

$$
L_{f} \dot{e}=\tilde{d}_{1}+A \tilde{I}_{1}+B \tilde{d}-K e
$$

where $\tilde{d}(t), \tilde{d}_{1}(t)$, and $\tilde{I}_{1}(t)$ are the estimation errors and observer error signal which are defined respectively by:

$$
\begin{gathered}
\tilde{d} \triangleq d-\hat{d} \\
\tilde{d}_{1} \triangleq d_{1}-\hat{d}_{1} \\
\tilde{I}_{1} \triangleq \hat{I}_{1}-I_{1} .
\end{gathered}
$$

Based on the subsequent stability analysis, the update laws of the system disturbances are defined as following: 


$$
\begin{gathered}
\dot{\hat{d}} \triangleq K_{d} B e \\
\hat{d}_{1}(t) \triangleq \operatorname{sat}_{\beta}\left(\hat{d}_{1}(t-T)\right)+K_{d 1} e(t)
\end{gathered}
$$

where $K_{d}, K_{d 1} \in \mathbb{R}^{+}$are control gains, $T$ is a known period of the grid side AC system, and $\operatorname{sat}_{\beta}($.$) is the saturation function which has an upper bound \beta$ and lower bound $-\beta$, where $\beta$ is a positive constant. Substituting (18) in (15), the error signal of the periodic disturbance is defined as:

$$
\begin{aligned}
\tilde{d}_{1}(t)=d_{1}-\hat{d}_{1} & =d_{1}(t-T)-\hat{d}_{1} \\
= & \operatorname{sat}_{\beta}\left(d_{1}(t-T)\right)-\operatorname{sat}_{\beta}\left(\hat{d}_{1}(t-T)\right)-K_{d 1} e(t) .
\end{aligned}
$$

\subsubsection{OBSERVER DESIGN}

To remove the need of the costly and noisy current sensor, the inverter current observer has been designed in this section. By taking the time derivative of the current observer error in (16), pre-multiplying by $L_{f}$, and substituting (6), the open loop observer error can be defined as:

$$
L_{f} \dot{\tilde{I}}_{1}=L_{f} \dot{\hat{I}}_{1}+R_{f} I_{1}+V_{o}-u V_{d c} .
$$

From (20) and based on the subsequent stability analysis, the observer $\hat{I}_{1}(t)$ is designed as:

$$
\dot{\hat{I}}_{1} \triangleq \frac{1}{L_{f}}\left[-R_{f} \hat{I}_{1}-V_{o}+u V_{d c}\right] .
$$

The closed loop system equation of the current observer error can be defined by substituting (21) into (20) as:

$$
L_{f} \dot{\tilde{I}}_{1}=-R_{f} \tilde{I}_{1} .
$$




\subsubsection{STABILITY ANALYSIS FOR THE INNER-VOLTAGE LOOP}

Theorem 1: The closed loop error equations defined in (13) and (22) ensure that the error signals in (8) and (16) are regulated as $e(t), \tilde{I}_{1}(t) \rightarrow 0$ as $t \rightarrow \infty$.

Proof: A non-negative Lyapunov function $V(t) \in \mathbb{R}$ is defined as:

$$
V \triangleq \frac{L_{f}}{2} e^{2}+\frac{L_{f}}{2} \tilde{I}_{1}^{2}+\frac{1}{2 k_{d}} \tilde{d}^{2}+\frac{1}{2 k_{d 1}} \int_{t-T}^{T}\left[\operatorname{sat}_{\beta}\left(d_{1}(\tau)\right)-\operatorname{sat}_{\beta}\left(\hat{d}_{1}(\tau)\right)\right]^{2} d \tau
$$

Taking the time derivative of (23) and then substituting the closed loop error signals from (13), (22) along with the disturbance update law from (17), the following expression can be obtained:

$$
\begin{gathered}
\dot{V}=-K e^{2}-R_{f} \tilde{I}_{1}^{2}-A e \tilde{I}_{1}+e \tilde{d}_{1}+\frac{1}{2 k_{d 1}}\left[\operatorname{sat}_{\beta}\left(d_{1}(\tau)\right)-\operatorname{sat}_{\beta}\left(\hat{d}_{1}(\tau)\right)\right]^{2} \\
-\frac{1}{2 k_{d 1}}\left[\operatorname{sat}_{\beta}\left(d_{1}(\tau-T)\right)-\operatorname{sat}_{\beta}\left(\hat{d}_{1}(\tau-T)\right)\right]^{2}
\end{gathered}
$$

Substituting (19) into (24), the expression in (25) is obtained:

$$
\begin{array}{r}
\dot{V}=-K e^{2}-R_{f} \tilde{I}_{1}^{2}-A e \tilde{I}_{1}+e \tilde{d}_{1}-\frac{1}{2 k_{d 1}}\left[\tilde{d}_{1}+K_{d 1} e\right]^{2} \\
+\frac{1}{2 k_{d 1}}\left[\operatorname{sat}_{\beta}\left(d_{1}(\tau)\right)-\operatorname{sat}_{\beta}\left(\hat{d}_{1}(\tau)\right)\right]^{2}
\end{array}
$$

After some mathematical simplification, and using the definition of $\tilde{d}_{1}(t)$ from (15), (25) can be rewritten as:

$$
\begin{gathered}
\dot{V}=-\left(\frac{K_{d 1}}{2}\right) e^{2}-R_{f} \tilde{I}_{1}^{2}-K e^{2}-A e \tilde{I}_{1} \\
+\frac{1}{2 K_{d 1}}\left\{\left[\operatorname{sat}_{\beta}\left(d_{1}(\tau)\right)-\operatorname{sat}_{\beta}\left(\hat{d}_{1}(\tau)\right)\right]^{2}-\left[d_{1}(t)-\hat{d}_{1}(t)\right]^{2}\right\} .
\end{gathered}
$$

By using the following property of the saturation function [45]: 


$$
(x-y)^{2} \geq\left(\operatorname{sat}_{\beta}(x)-\operatorname{sat}_{\beta}(y)\right)^{2}
$$

The expression in the (26) can be upper bounded as:

$$
\dot{V} \leq-\left(K+\frac{K_{d 1}}{2}\right)\left|e^{2}\right|-R_{f}\left|\tilde{I}_{1}^{2}\right|+A|e|\left|\tilde{I}_{1}\right|
$$

By applying triangle inequality, (28) can be further upper bounded as:

$$
\dot{V} \leq-\left(K+\frac{K_{d 1}}{2}+\frac{A}{2}\right)\left|e^{2}\right|-\left(R_{f}+\frac{A}{2}\right)\left|\tilde{I}_{1}^{2}\right|
$$

From (23) and (29) it is clear that $e(t), \tilde{I}_{1} \in \mathcal{L}_{\infty} \cap \mathcal{L}_{2}$ and $\tilde{d} \in \mathcal{L}_{\infty}$. Based on (8) and Assumption 8, one can see that $V_{o} \in \mathcal{L}_{\infty}$. From the definition of the $s a t_{\beta}($.$) function and$ $e(t) \in \mathcal{L}_{\infty}$, one can use (18) to conclude that $\hat{d}_{1} \in \mathcal{L}_{\infty}$. Then, from $\hat{d}_{1}, e \in \mathcal{L}_{\infty}$ and (19), it is clear that $\tilde{d}_{1} \in \mathcal{L}_{\infty}$. By using (15) and the above it is easy to say that $d_{1} \in \mathcal{L}_{\infty}$. From (10) and Assumption 6, it is easy to say that $\dot{V}_{\text {oref }} \in \mathcal{L}_{\infty}$. Since $\tilde{d} \in \mathcal{L}_{\infty}$ and by using Assumption 3, from (14) we can deduce that $\hat{d} \in \mathcal{L}_{\infty}$. From (16), $V_{o}, \tilde{I}_{1} \in \mathcal{L}_{\infty}$, and Assumption 7, we can see that $\hat{I}_{1} \in \mathcal{L}_{\infty}$. From (22) it is easy to show that $\dot{\tilde{I}}_{1} \in \mathcal{L}_{\infty}$. From (12) and using $V_{o}, \hat{d}, e, \hat{I}_{1}, \hat{d}_{1} \in \mathcal{L}_{\infty}$, we can conclude that $u(t) \in \mathcal{L}_{\infty}$. Now from (21) we can see that $\dot{\hat{I}}_{1} \in \mathcal{L}_{\infty}$. From (13) and by using the above results it is obvious that $\dot{e}(t) \in$ $\mathcal{L}_{\infty}$. Therefore, all signals in the closed loop are bounded. Since $e(t), \tilde{I}_{1}(t) \in \mathcal{L}_{\infty} \cap \mathcal{L}_{2}$ and $\dot{e}(t), \dot{\tilde{I}}_{1}(t) \in \mathcal{L}_{\infty}$ Barbalat's Lemma [46] can be utilized to prove that $e(t), \tilde{I}_{1}(t) \rightarrow$ 0 as $t \rightarrow \infty$. Thus, completing the proof of Theorem 1 .

\subsubsection{OUTER-LOOP CURRENT CONTROL DESIGN}

Figure. 3 shows the grid side system which has been used in this section to design the outer-loop current controller. The main objective of the outer-loop current controller is 
exchanging a clean current with the grid by regulating the grid current $I_{2}(t)$, hence $I_{2}(t) \rightarrow$ $I_{2 r e f}(t)$ as $t \rightarrow \infty$.

As mentioned previously, to design the controller in the outer-loop the objective of the controller in the inner-loop is assumed to have already been met. To ensure this condition is meet, a supervisory control on the inner-loop controller is necessary before activating the outer-loop controller as shown in Fig.2.4.

To facilitate the outer-loop current controller design, equation (3) could be rewritten as:

$$
\begin{gathered}
L_{g} \dot{I}_{2}=-R_{g} I_{2}+V_{i} . \\
V_{i}=V_{o}-V_{g} .
\end{gathered}
$$

In (30), $V_{i}(t)$ must be designed to ensure that the grid current $I_{2}(t)$ is tracking a given reference grid current $I_{2 r e f}(t)$. To guarantee unity power factor at the grid side, the reference grid current should be completely sinusoidal and in phase with the grid voltage:

$$
I_{2 r e f} \triangleq I_{2 p} \sin \theta
$$

where $I_{2 p}$ is the peak value of the reference grid current which is selected by the user. Also, $\theta$ is the phase angle of the grid voltage. This angle has been estimated based on a second

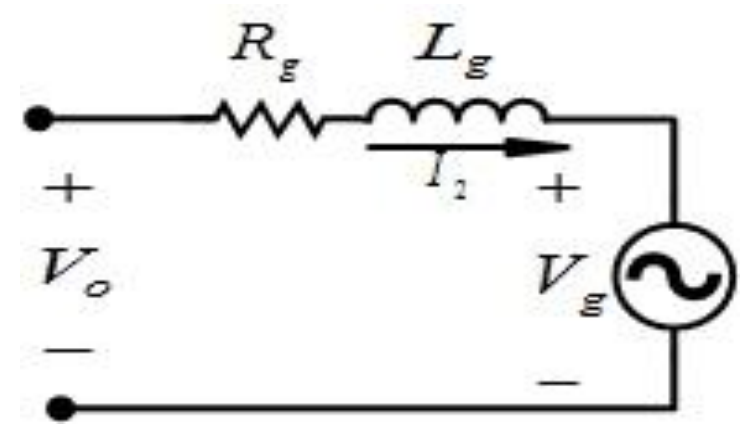

Figure 0.3 Grid side system for outer-loop current controller

order generalized integrator to create an orthogonal signal generator-based phase-locked 
loop (SOGI-OSG PLL). We chose this method based on the results in [47], although other PLL schemes could also be used.

To start the outer-loop control design process, the tracking error signal $\eta(t) \in \mathbb{R}$ is defined as:

$$
\eta \triangleq I_{2 r e f}-I_{2}
$$

After taking the time derivative of (33), pre-multiplying by $L_{g}$ and substituting (30) in the result, the open loop error dynamic of $\eta(t)$ is obtained as:

$$
L_{g} \dot{\eta}=L_{g} \dot{I}_{2 r e f}+R_{g} I_{2}-V_{i}
$$

Based on the subsequent stability analysis and the form in (34), the control law from the outer-loop $V_{i}(t)$ is designed as follows:

$$
V_{i} \triangleq \hat{L}_{g} \dot{I}_{2 r e f}+\hat{R}_{g} I_{2}+K_{g} \eta
$$

where $K_{g} \in \mathbb{R}^{+}$is a control gain, and $\hat{L}_{g}, \hat{R}_{g}$ are the estimation of the unknown parameters of the grid impedance. Based on the stability analysis the update laws of the unknown parameters are defined by:

$$
\begin{gathered}
\dot{\hat{L}}_{g}=K_{L} \eta \dot{I}_{2 r e f} \\
\dot{\hat{R}}_{g}=K_{R} \eta I_{2}
\end{gathered}
$$

where $K_{L}, K_{R}$ are positive gains. The control signal defined in (35) is substituted in (30) to get the following closed loop error system for $\eta(t)$ as:

$$
L_{g} \dot{\eta}=\tilde{L}_{g} \dot{I}_{2 r e f}+\tilde{R}_{g} I_{2}-K_{g} \eta
$$

where $\tilde{L}_{g}$ and $\tilde{R}_{g}$ are estimation errors which defined by: 


$$
\begin{gathered}
\tilde{L}_{g} \triangleq L_{g}-\hat{L}_{g} \\
\tilde{R}_{g} \triangleq R_{g}-\hat{R}_{g} .
\end{gathered}
$$

Remark: The reference voltage trajectory $V_{o_{\text {ref }}}$ can be obtained from (31) and (35) under the assumption that the inner-loop is already in the steady state as:

$$
V_{o_{\text {ref }}}=\widehat{L}_{g} \dot{I}_{2 r e f}+\hat{R}_{g} I_{2}+V_{g}+K_{g} \eta
$$

\subsubsection{STABILITY ANALYSIS FOR THE OUTER-LOOP}

Theorem 2: The closed loop error equation defined in (38) ensures that the error signal in (33) is regulated as:

$$
\eta(t) \rightarrow 0 \text { as } t \rightarrow \infty
$$

Proof: A non-negative Lyapunov function $S(t) \in \mathbb{R}$ is defined as:

$$
S \triangleq \frac{L_{g}}{2} \eta^{2}+\frac{1}{2 k_{L}} \tilde{L}_{g}{ }^{2}+\frac{1}{2 k_{R}} \tilde{R}_{g}{ }^{2}
$$

Taking the time derivative of (42), the result is as follows:

$$
\dot{S}=\eta\left(L_{g} \dot{\eta}\right)-\frac{1}{k_{L}} \tilde{L}_{g} \dot{\hat{L}}_{g}-\frac{1}{k_{R}} \tilde{R}_{g} \dot{\hat{R}}_{g}
$$

Substituting the closed loop error dynamic and the parameters update laws from (38), (36) and (37), the expression in (44) is obtained:

$$
\dot{S}=-K_{g} \eta^{2}
$$

From (42) and (44) it is clear that $\eta(t) \in \mathcal{L}_{\infty} \cap \mathcal{L}_{2}$ and $\tilde{L}_{g}, \tilde{R}_{g} \in \mathcal{L}_{\infty}$. From Assumption 5, and by using (33) along with $\eta(t) \in \mathcal{L}_{\infty}$, it is easy to show that $I_{2} \in \mathcal{L}_{\infty}$. From (39), (40), $\tilde{L}_{g}, \tilde{R}_{g} \in \mathcal{L}_{\infty}$, and Assumption 4 , one can say that $\hat{L}_{g}, \hat{R}_{g} \in \mathcal{L}_{\infty}$. Now, we can see that all 
signals in (35) are bounded, so it is clear to say that $V_{i} \in \mathcal{L}_{\infty}$. Using (41), $V_{g}(t) \in \mathcal{L}_{\infty}$ and above results, it is easy to say that $V_{o_{\text {ref }}} \in \mathcal{L}_{\infty}$. This is motivation for Assumption 8. From (38) and by using the above results it is obvious that $\dot{\eta}(t) \in \mathcal{L}_{\infty}$. Therefore, all signals in the closed loop systems are bounded. Since $\eta(t) \in \mathcal{L}_{\infty} \cap \mathcal{L}_{2}$ and $\dot{\eta}(t) \in \mathcal{L}_{\infty}$ Barbalat's Lemma [46] can be utilized to prove that $\eta(t) \rightarrow 0$ as $t \rightarrow \infty$. At this point, we have completed the proof of the Theorem 2 .

\subsection{SIMULATION RESULTS}

To evaluate the performance of the proposed controller, the PLECS software is used to model the instantaneous circuit dynamics of the system and the control scheme. The proposed control structure is shown in Fig.4. The system parameters and the controller gains are listed in Table I.

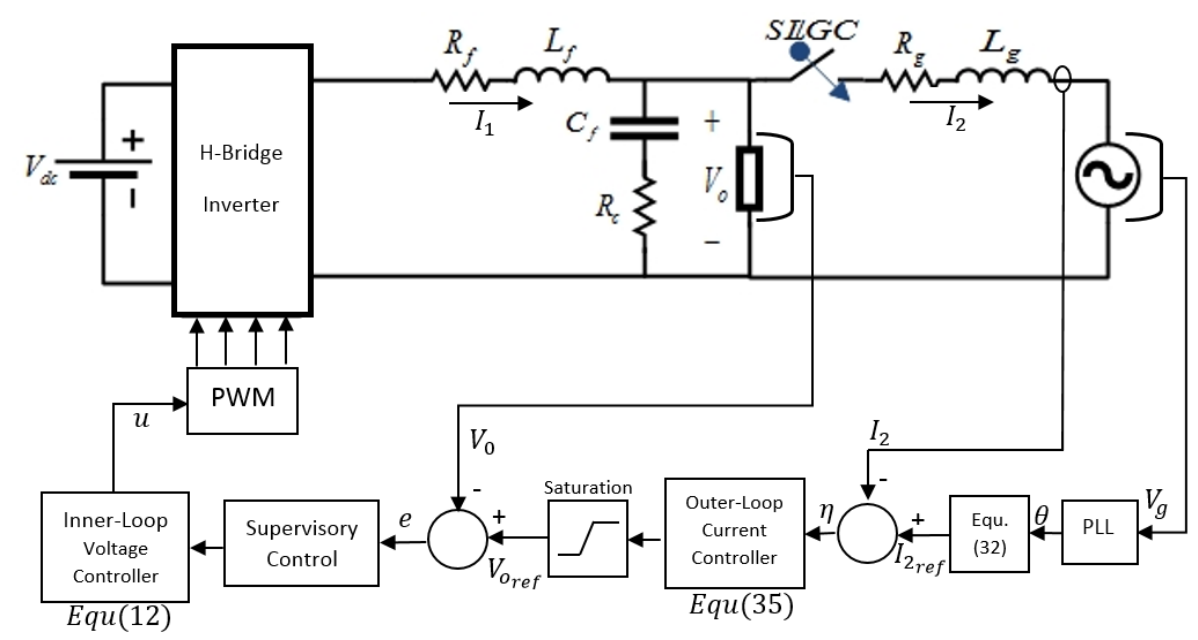

Figure 0.4 Proposed cascaded control structure 
Table 0.1 System Parameters

\begin{tabular}{|c|c|c|c|c|c|}
\hline Parameter & Value & Units & Gain & Value & Units \\
\hline \multicolumn{3}{|c|}{ Inverter Parameters } & \multicolumn{3}{|c|}{ Controller Gains } \\
\hline$L_{f}$ & 150 & $\mu H$ & $K$ & 1 & --- \\
\hline$R_{f}$ & 0.045 & $\Omega$ & $K_{d}$ & 1000 & --- \\
\hline \multirow[b]{2}{*}{$C_{f}$} & \multirow{2}{*}{22} & \multirow{2}{*}{$\mu F$} & $K_{d 1}$ & 0.01 & --- \\
\hline & & & $K_{g}$ & 3 & --- \\
\hline \multirow[b]{2}{*}{$R_{c}$} & \multirow{2}{*}{0.1} & \multirow{2}{*}{$\Omega$} & $K_{L}$ & 0.006 & --- \\
\hline & & & $K_{R}$ & 1000 & --- \\
\hline$V_{d c}$ & 42 & $V$ & $f_{s w}$ & 20 & $K H_{z}$ \\
\hline \multicolumn{6}{|c|}{ Grid Parameters } \\
\hline$L_{g}$ & 450 & $\mu H$ & $V_{g}$ & 15 & $V_{\text {peak }}$ \\
\hline$R_{g}$ & 0.135 & $\Omega$ & $f_{g}$ & 60 & $\mathrm{~Hz}$ \\
\hline \multicolumn{6}{|c|}{ Loads Parameters } \\
\hline Resistive & \multicolumn{5}{|c|}{$\mathrm{R}=20 \Omega$} \\
\hline Inductive & \multicolumn{5}{|c|}{$\mathrm{R}=20 \Omega, \mathrm{L}=32 \mathrm{mH}$} \\
\hline Nonlinear & \multicolumn{5}{|c|}{ Single phase rectifier with $\mathrm{R}=50 \Omega, \mathrm{C}=680 \mu \mathrm{F}$} \\
\hline
\end{tabular}

\subsubsection{STEADY-STATE PERFORMANCE}

To check the steady-state performance of the proposed controller, the following tests have been performed:

\subsubsection{RESISTIVE LOCAL LOAD TEST}

Fig.5 and Fig.6 show a very good tracking performance of the proposed controller for the local load voltage $V_{o}(t)$ and the grid current $I_{2}(t)$. Fig.7 shows the control signal $u(t)$. Also, Fig8. shows the convergence of the updated law of the grid inductance and resistance. The observer performance has been shown in Fig 9. 


\subsubsection{INDUCTIVE LOCAL LOAD TEST}

In Fig.10, the grid voltage, grid current, local load voltage and load current are shown together in the same plot in order to show unity power factor at the grid side in the presence of the inductive local load.

\subsubsection{NONLINEAR LOCAL LOAD TESTS}

The grid voltage, grid current, and the load current are shown in the Fig.11. The Total Harmonic Distortion (THD) for output voltage, grid current, and output current are $0.73 \%$, $2.35 \%, 110.6 \%$, respectively. It is seen that with high distorted nonlinear load current, the local load voltage and the injected current to the grid remain clean with very low distortion. Also, it can be seen that the power factor at the grid side is still unity in the presence of the nonlinear local load. The percentage of maximum steady state errors for all tests are summarized in Table II to show the effectiveness of the proposed controller in terms of the steady state error (SSE).

\subsubsection{TRANSIENT-STATE PERFORMANCE}

To check the transient-state performance of the proposed controller, the following simulations have been done:

\subsubsection{STEP CHANGE IN THE REFRENCE GRID CURRENT}

The inverter was connected to the grid without local load and with the peak reference grid current $I_{2 p}=2[\mathrm{~A}]$. At the time $\mathrm{t}=0.06$ seconds, the reference current is increased to $3 \mathrm{~A}$. Fig.12 shows a very good tracking performance of the proposed controller. 


\subsubsection{STEP CHANGE IN THE RESISTIVE LOCAL LOAD}

Fig.13 shows the system response for the proposed controller when the resistive local load changed from $\mathrm{R}=20 \Omega$ to $\mathrm{R}=100 \Omega$ at $\mathrm{t}=0.06$ seconds and back to the original value at $t=0.14$ seconds. From the figure it is easy to see the robustness of the proposed controller since there is no significant fluctuations in the grid current and load voltage.

\subsubsection{SEAMLESS TRANSITION BETWEEN GA AND SA MODES}

The system responses during the transition between the stand-alone mode and the gridconnected mode are shown in Fig.14 and Fig.15 while the inverter supplies resistive local load. It can be seen there are no oscillations in the grid current within the transition. Thus, a seamless transition in the output voltage has been achieved by the proposed controller. Based on these results, we can conclude that the proposed controller is working well in the two modes without the need to change the structure of the controller, and so the system can transfer between these two modes smoothly.

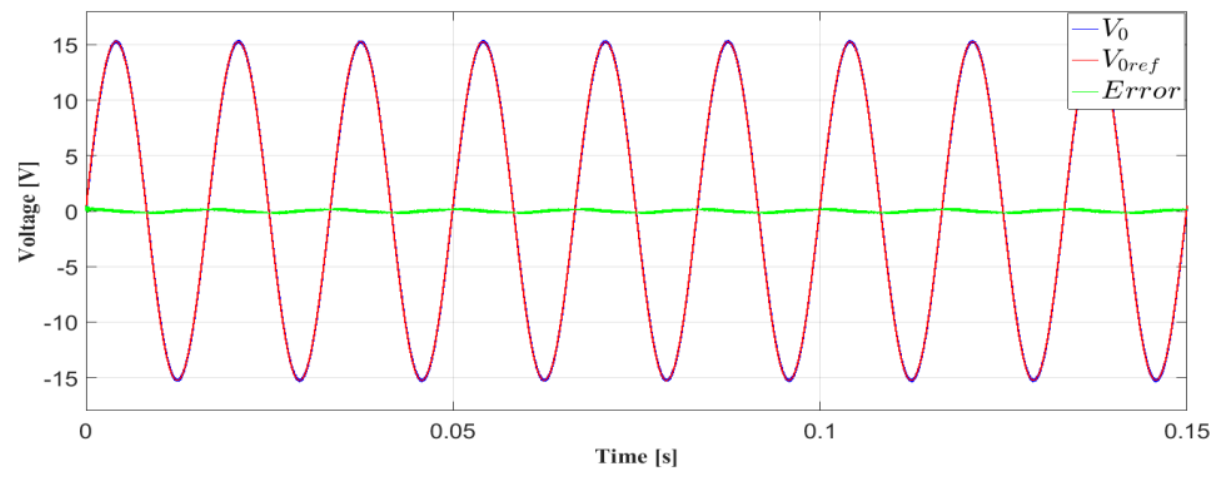

Figure 0.5 Tracking response of the output voltage for resistive local load. 


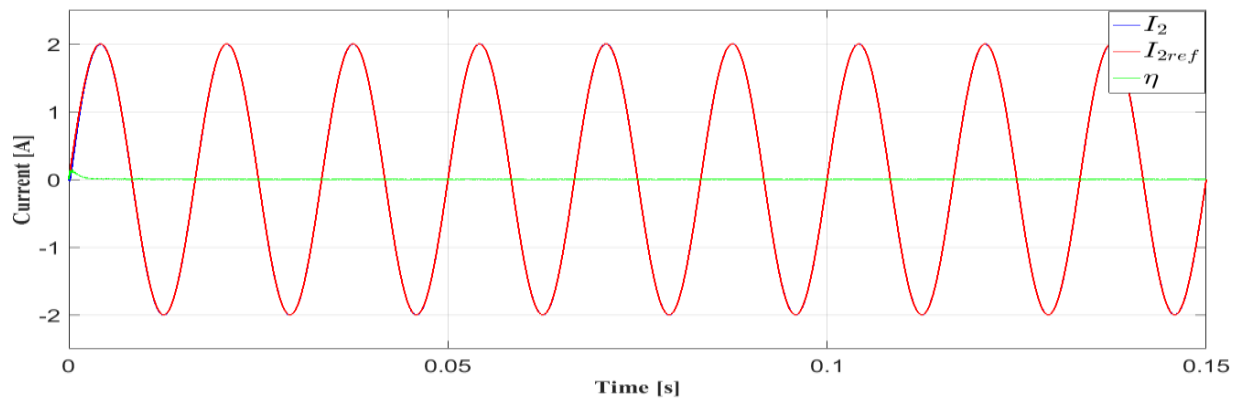

Figure 0.6 Tracking response of the grid current under resistive local load.

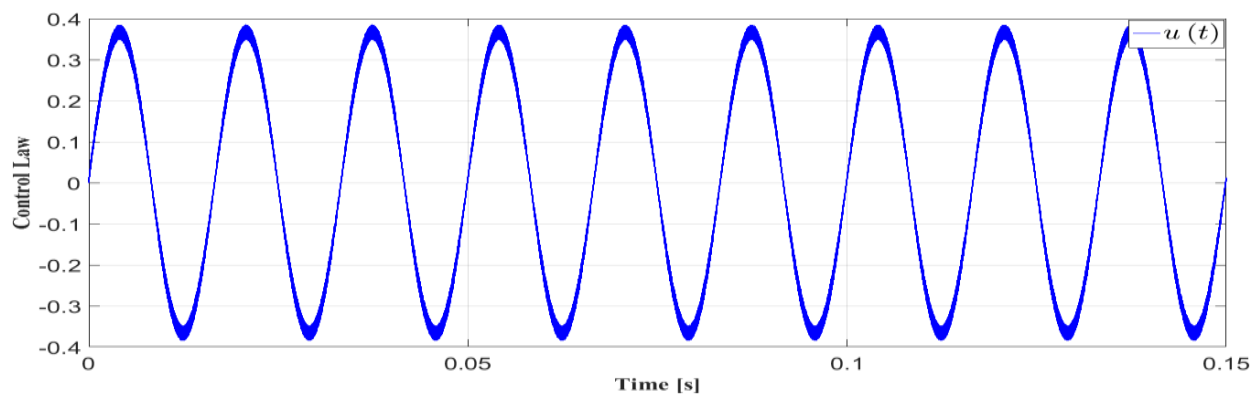

Figure 0.7 Control Signal $u(t)$ for resistive local load. 

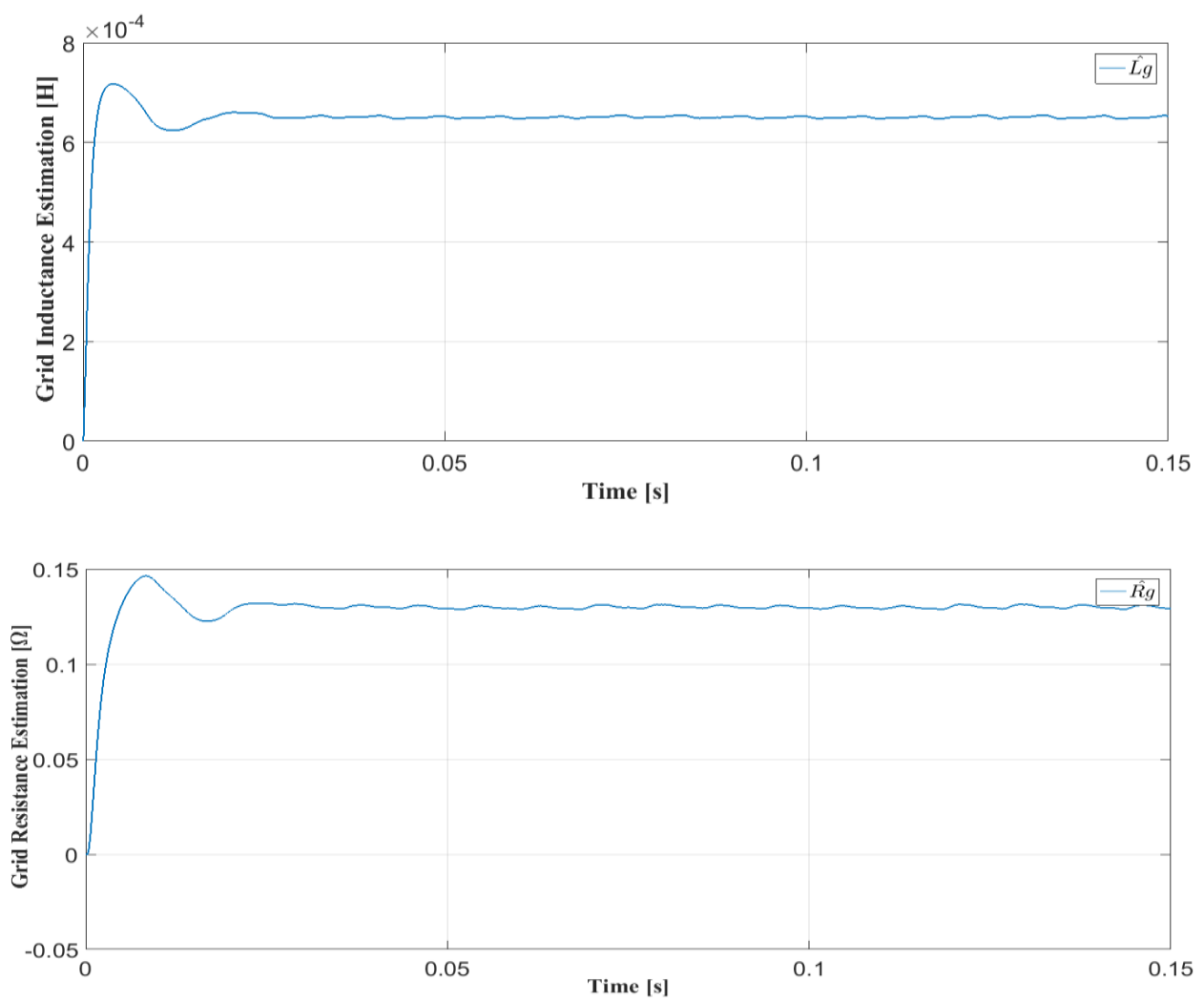

Figure 0.8 :Convergence response of the grid impedance $L_{g}, R_{g}$.

Table 0.2 Steady State Errors for cascaded controller with different types of loads

\begin{tabular}{|c|r|r|r|}
\hline LSE Type & $\begin{array}{c}\text { Resistive } \\
\text { load }\end{array}$ & $\begin{array}{c}\text { Inductive } \\
\text { Load }\end{array}$ & $\begin{array}{c}\text { Nonlinear } \\
\text { Load }\end{array}$ \\
\hline$e(t)$ & $1.6 \%$ & $1.6 \%$ & $4.58 \%$ \\
\hline$\eta(t)$ & $0.1 \%$ & $0.13 \%$ & $6.3 \%$ \\
\hline$\tilde{I}_{1}(t)$ & $3 \%$ & $4.9 \%$ & $8.85 \%$ \\
\hline
\end{tabular}




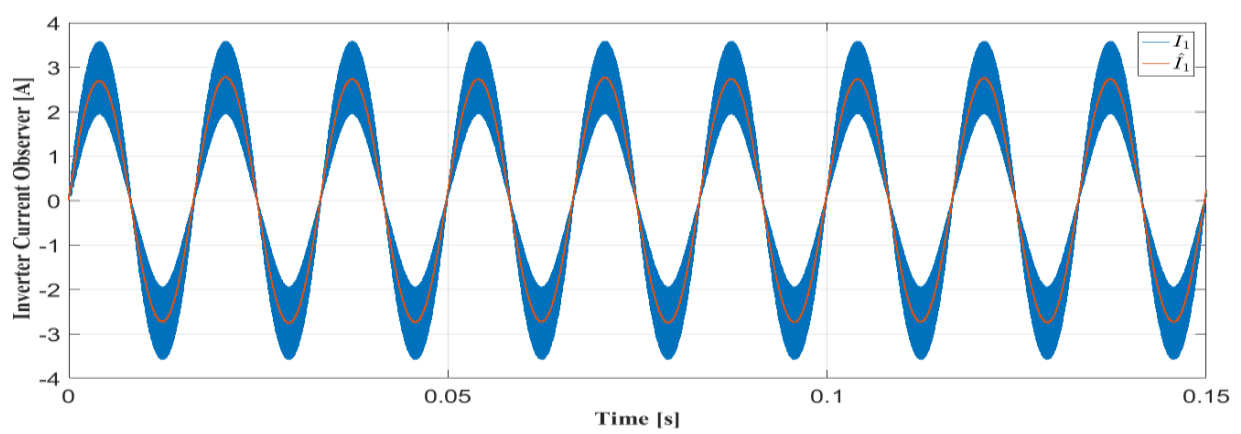

Figure 0.9 Inverter current observer response.

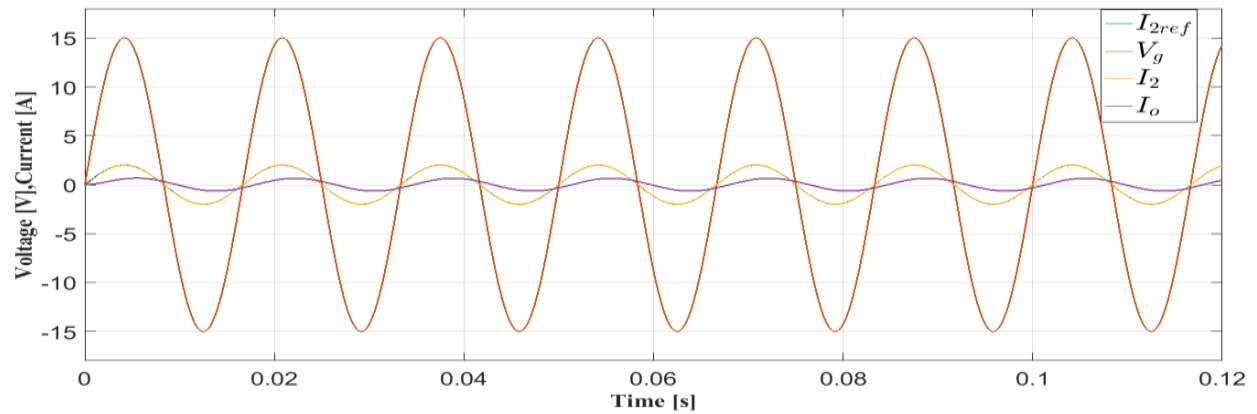

Figure 0.10 System responses of the inductive local load.

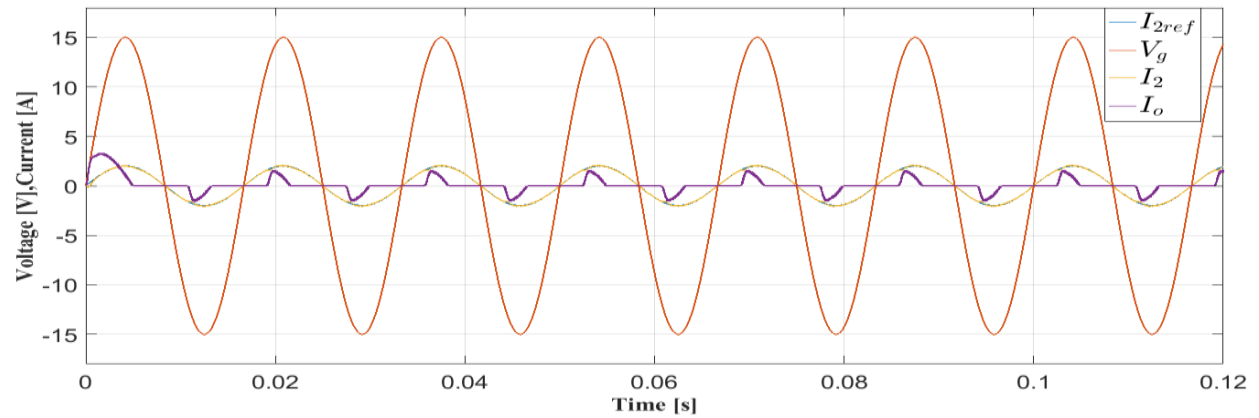

Figure 0.11 System responses of the nonlinear local load. 


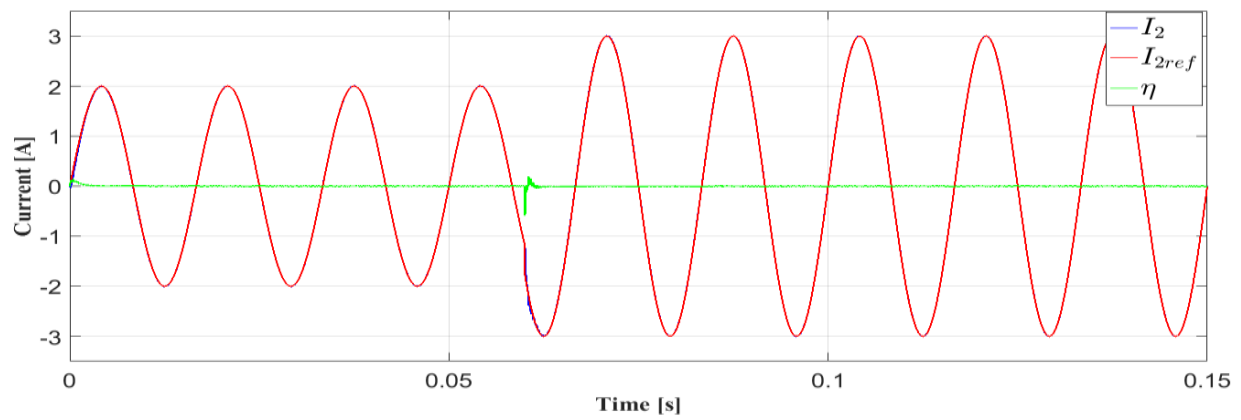

Figure 0.12 Tracking response of the grid current for step change in the reference current.

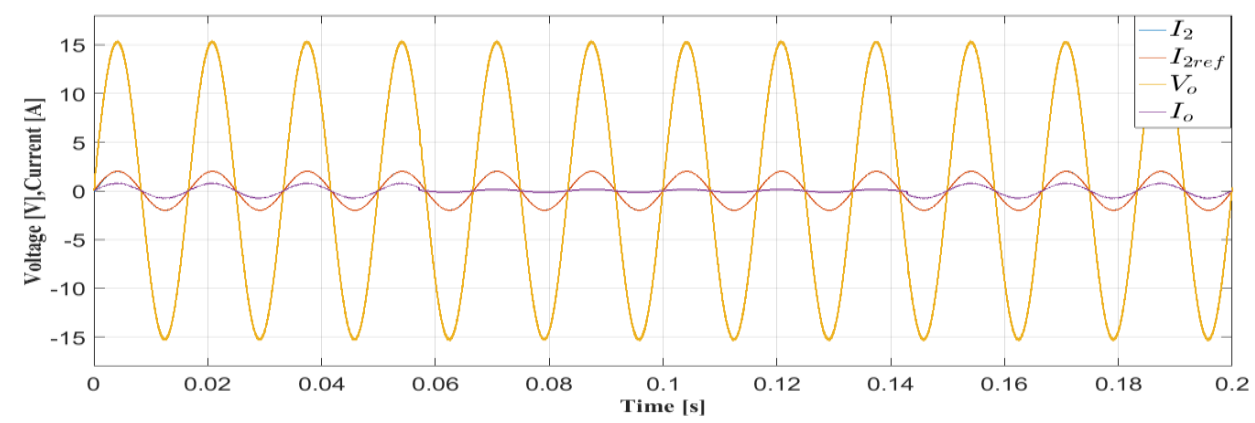

Figure 0.13 Tracking response of the grid current under load changing.

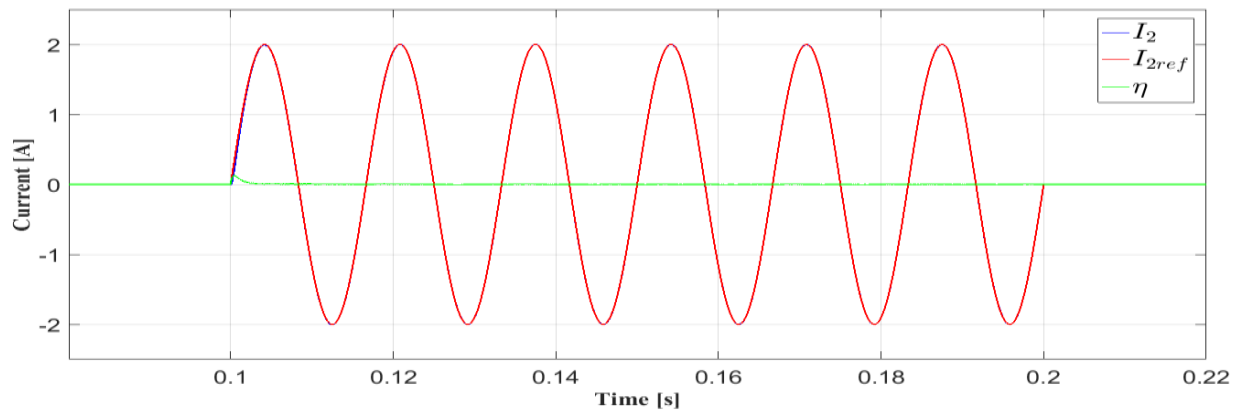

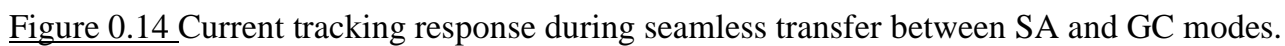




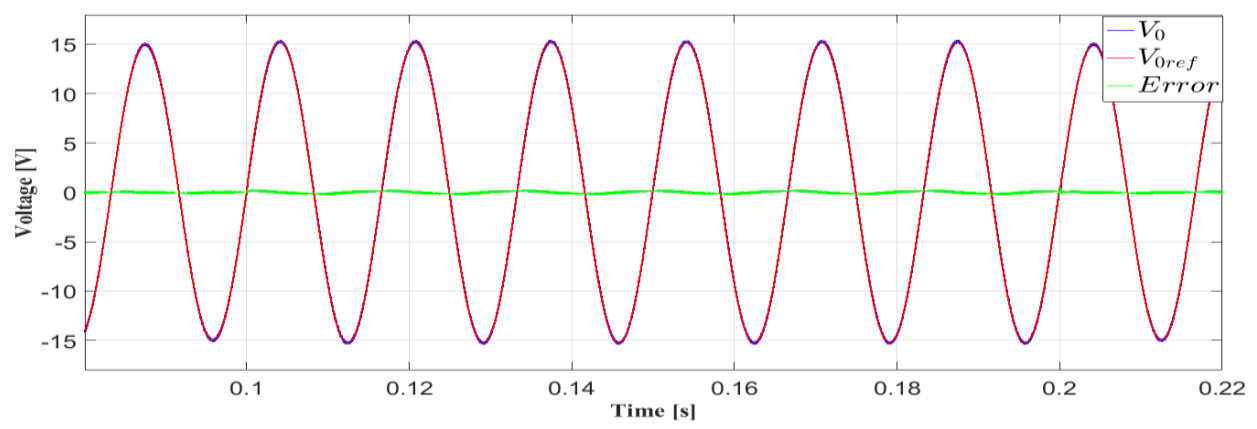

Figure 0.15 Output voltage response during seamless transfer between SA and GC modes.

\subsection{SUMMARY}

A cascaded nonlinear controller has been proposed in this chapter for a single-phase gridconnected inverter. The proposed controller improves the quality of the local load voltage and the grid current simultaneously. This controller includes a current outer-loop and a voltage inner-loop. These two loops are designed based on a Lyapunov based stability analysis. The design procedure has been accomplished by using a learning control approach to estimate a bounded periodic disturbance. Also, a current observer has been designed to estimate the inductor current of the inverter. The grid impedance is considered as unknown parameter. This stability analysis shows that the system is stable and all signals in the closed loop systems are bounded. The controller performance has been tested in the steadystate and in the transient-state via simulation. These simulation results demonstrate that the proposed controller has excellent tracking performance and is robust against changes in operating conditions. Also, a seamless transition between standalone and grid-connected modes has been achieved. 


\section{CHAPTER 3}

\section{BACKSTEPPING CONTROL DESIGN}

In this chapter the backstepping control theory has been utilized to design a nonlinear controller for the single-phase grid connected inverter system. This approach has been proposed to overcome the shortfalls of the cascaded control scheme in the previous chapter. In general, the main disadvantage of the cascaded control schemes is that they require the objective in the inner control loop to be met before activating the outer control loop, and to ensure that a supervisory control is needed in the cascaded approaches. Moreover, cascaded control assumes that the stability of the two control loops are independent.

The main control objective in this chapter is to simultaneously improve the quality of the local load voltage while injecting clean current to the grid. Moreover, the proposed approach ensures the seamless transfer between grid-connected and stand-alone operation modes without adjusting the controller structure. The proposed controller is validated through a Lyapunov stability analysis. An instantaneous circuit simulation in PLECS software was utilized to validate the proposed control scheme.

The remainder of this chapter is organized as follows. Third order mathematical model of the single-phase grid-connected inverter with LC filter is presented in Section 3.1. In Section 3.2, this model is then used to develop the proposed backstepping control scheme. A Lyapunov stability analysis is performed in Section 3.3. The simulation results in Section 3.4 validate the controller development. Concluding remarks are provided in Section 3.5. 


\subsection{SYSTEM MODEL}

The system in Fig.3.1 consists of the following elements: DC power source $V_{d c}$, H-bridge voltage source inverter (VSI), LC filter $L_{f}, C_{f}$. The grid is represented by an ac voltage source $V_{g}$. The grid interface inductor is represented by $L_{g}$ which allows to connect the grid to the inverter system. A local load has been connected in parallel to the capacitor $C_{f}$. By applying KVL and KCL to the average model of the inverter system, the mathematical differential equations representing the system are obtained as:

$$
\begin{gathered}
L_{f} \dot{I}_{1}=V_{d c}\left(D+d_{0}\right)-V_{o} \\
C_{f} \dot{V}_{o}=I_{1}-I_{2}-I_{o} \\
L_{g} \dot{I}_{2}=V_{o}-V_{g}
\end{gathered}
$$

where $I_{1}(t), V_{o}(t), I_{2}(t) \in \mathbb{R}$ are the inductor output current, output (capacitor) voltage, and injected current to the grid, respectively. $I_{o} \in \mathbb{R}$ is the current of the local load. $D(t) \in$ $\mathbb{R}$ is duty ratio of the switching signal. $d_{0} \in \mathbb{R}$ is an unknown disturbance.

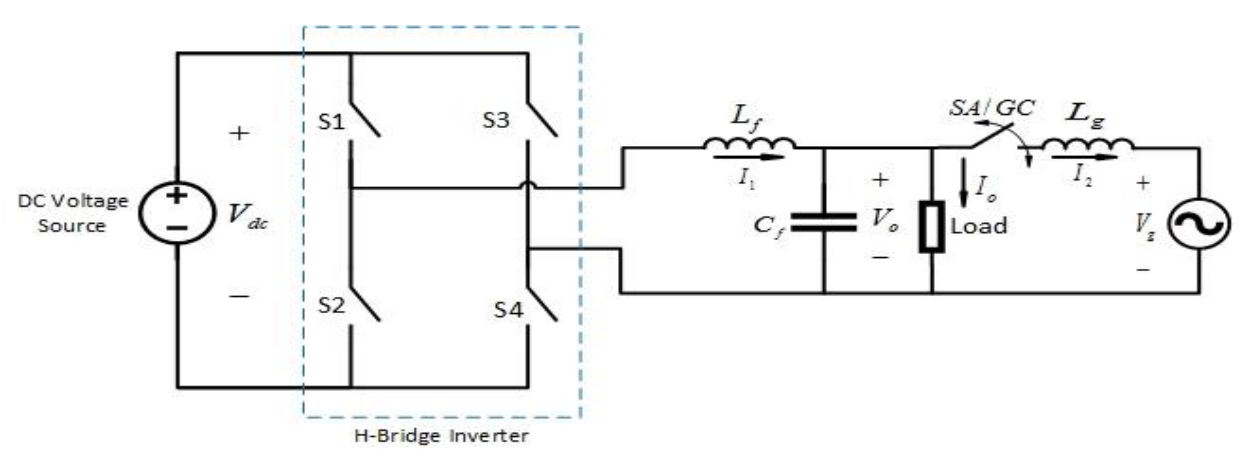

Figure 0.1 Single-phase grid-connected inverter system 


\subsection{CONTROL DEVELOPMENT}

The voltage control objective of the proposed controller is to maintain low THD of the local load voltage $V_{o}(t)$ regardless of the type of load and achieve the reference output voltage trajectory $V_{\text {oref }}(t)$ which will be designed later, hence $V_{o}(t) \rightarrow V_{\text {oref }}(t)$ as $t \rightarrow$ $\infty$. Also, the current control objective is to exchange clean current with the grid by ensuring that the grid current $I_{2}(t)$ is tracking a given reference current $I_{2_{r e f}}(t)$, hence $I_{2}(t) \rightarrow I_{2_{\text {ref }}}(t)$ as $t \rightarrow \infty$. To guarantee unity power factor at the grid side, the reference grid current should be completely sinusoidal and in phase with the grid voltage:

$$
I_{2 \text { ref }} \triangleq I_{2 p} \sin \theta
$$

where $I_{2 p}$ is the peak value of the reference grid current which is selected by the user, and $\theta$ is the phase angle of the grid voltage. This angle has been estimated based on a second order generalized integrator to create an orthogonal signal generator-based phase-locked loop (SOGI-OSG PLL) [47]. It is possible to use other PLL schemes.

The subsequent development of the proposed controller is based upon the following assumptions:

Assumption 1: $L_{g}, L_{f}, C_{f}, V_{d c}$ are known, constant system parameters.

Assumption 2: The output voltage $V_{o}(t)$ and filter inductor current $I_{1}(t)$ are measurable.

Assumption 3: The unknown disturbance $d_{0}$ is bounded and slowly time-varying, hence $\dot{d}_{0}(t) \approx 0$ 
Assumption 4: The output current $I_{o}$ is bounded and unknown and assumed to be slowly time varying.

Assumption 5: The grid voltage is bounded and differentiable defined as $V_{g}(t)=V_{m} \sin \theta$, where $V_{m}$ is the voltage magnitude and $\theta$ is the grid phase angle.

In order to meet the current and voltage tracking objectives, the following error signals are defined:

$$
\begin{aligned}
& e_{2} \triangleq I_{2_{\text {ref }}}-I_{2} \\
& \eta \triangleq V_{o_{\text {ref }}}-V_{o} .
\end{aligned}
$$

To proceed with the control development, the following auxiliary error signals are defined as:

$$
\begin{aligned}
& e_{1} \triangleq I_{1}^{*}-I_{1} \\
& \tilde{I}_{o}=I_{o}-\hat{I}_{o}
\end{aligned}
$$

where $I_{1}^{*} \in \mathbb{R}$ is an auxiliary control signal which will be designed later, and $\hat{I}_{o} \in \mathbb{R}$ is the estimation of the output load current.

Taking the time derivative of (5), and substituting $\dot{I}_{2}$ and $V_{o}$ from (3) and (6), the open loop error dynamics of $e_{2}(t)$ is obtained after pre-multiplying by $L_{g}$ as follows:

$$
L_{g} \dot{e}_{2}=L_{g} \dot{I}_{2 e f}-V_{o r e f}+\eta+V_{g}
$$

The reference voltage trajectory can be designed to regulate $I_{2}$ based on (9) as:

$$
V_{o_{r e f}} \triangleq L_{g} \dot{I}_{2 r e f}+V_{g}+K_{2} e_{2}
$$


where $K_{2} \in \mathbb{R}^{+}$is a control gain. After substituting (10) into (9), the closed loop error system for $e_{2}(t)$ is obtained:

$$
L_{g} \dot{e}_{2}=\eta-K_{2} e_{2}
$$

Taking the time derivative of (10) and using (9), $\dot{V}_{\text {ref }}$ can be obtained for later use:

$$
\dot{V}_{o_{r e f}}=L_{g} \ddot{I}_{2 r e f}+\dot{V}_{g}+\frac{K_{2}}{L_{g}}\left(\eta-K_{2} e_{2}\right) \text {. }
$$

By taking the time derivative of (6) and using (2), (7), (8) and (12) the open loop error dynamics of $\eta(t)$ can be obtained as:

$$
C_{f} \dot{\eta}=C_{f} L_{g} \ddot{I}_{2 r e f}+C_{f} \dot{V}_{g}+\frac{C_{f} K_{2}}{L_{g}}\left(\eta-K_{2} e_{2}\right)-I_{1}^{*}+e_{1}+I_{2}+\tilde{I}_{o}+\hat{I}_{o} .
$$

From (14) and motivated by subsequent stability analysis, the auxiliary control signal $I_{1}^{*}$ can be designed to regulate the output voltage as follows:

$$
I_{1}^{*} \triangleq C_{f} L_{g} \ddot{I}_{2 r e f}+C_{f} \dot{V}_{g}+I_{2}+\hat{I}_{o}+K_{\eta} \eta
$$

where $K_{\eta} \in \mathbb{R}^{+}$is a control gain and $\hat{I}_{o}$ is the estimation of the output current with update law obtained from the subsequent stability analysis as:

$$
\hat{\hat{I}}_{o}=k_{0}\left[\eta+\frac{L_{f} K_{\eta}}{C_{f}} e_{1}\right]
$$

where $K_{0} \in \mathbb{R}^{+}$is a gain. By substituting the auxiliary control law from (15) into the open loop dynamics of $\eta(t)$ (14), the closed loop dynamics of $\eta(t)$ is obtained as follows:

$$
C_{f} \dot{\eta}=-\frac{C_{f} K_{2}^{2}}{L_{g}} e_{2}+e_{1}+\tilde{I}_{o}-B \eta
$$

where $B=\left(K_{\eta}-\frac{C_{f} K_{2}}{L_{g}}\right)$. Taking the time derivative of (15), using (3) and (17), $\dot{I}_{1}^{*}$ is obtained for later use: 


$$
\dot{I}_{1}^{*}=C_{f} L_{g} \dddot{I}_{2 r e f}+C_{f} \ddot{V}_{g}+\frac{1}{L g}\left(V_{o}-V_{g}\right)+\dot{\hat{I}}_{o}+\frac{K_{\eta}}{C_{f}}\left(-\frac{C_{f} K_{2}^{2}}{L_{g}} e_{2}+e_{1}+\tilde{I}_{o}-B \eta\right)
$$

By taking the time derivative of (7), pre-multiplying by $L_{f}$ and using (1) and (18), the open loop error dynamics of $e_{1}(t)$ is obtained as:

$$
\begin{aligned}
L_{f} \dot{e}_{1}= & L_{f} C_{f} L_{g} \dddot{I}_{2 r e f}+L_{f} C_{f} \ddot{V}_{g}-\frac{L_{f}}{L_{g}} V_{g}+\left(1+\frac{L_{f}}{L g}\right) V_{o}+L_{f} \dot{\hat{I}}_{o} \\
& +\frac{L_{f} K_{\eta}}{C_{f}}\left(-\frac{C_{f} K_{2}^{2}}{L_{g}} e_{2}+e_{1}+\tilde{I}_{o}-B \eta\right)-V_{d c}\left(D+d_{0}\right) .
\end{aligned}
$$

From (19) and motivated by the subsequent stability analysis the control law is designed as:

$$
D \triangleq \frac{1}{V_{d c}}\left[\begin{array}{c}
L_{f} C_{f} L_{g} \dddot{I}_{2 r e f}+L_{f} C_{f} \ddot{V}_{g}-\frac{L_{f}}{L_{g}} V_{g}+\left(1+\frac{L_{f}}{L_{g}}\right) V_{o}+L_{f} \dot{\hat{I}}_{o}+\eta \\
+\frac{L_{f} K_{\eta}}{C_{f}}\left(-\frac{C_{f} K_{2}^{2}}{L_{g}} e_{2}+e_{1}-B \eta\right)+K_{1} e_{1}-V_{d c} \hat{d}_{0}
\end{array}\right]
$$

where $K_{1} \in \mathbb{R}^{+}$is a control gain and $\hat{d}_{0}$ is the disturbance estimation defined by the following estimation error and update law:

$$
\begin{gathered}
\tilde{d}_{0} \triangleq d_{0}-\hat{d}_{0} \\
\dot{\hat{d}}_{0} \triangleq-k_{d} V_{d c} e_{1}
\end{gathered}
$$

where $k_{d} \in \mathbb{R}^{+}$is the estimation gain. Substituting the control law from (20) into (19), the following closed loop error dynamics of $e_{1}(t)$ can be obtained as follows:

$$
L_{f} \dot{e}_{1}=\frac{L_{f} K_{\eta}}{C_{f}} \tilde{I}_{o}-\eta-V_{d c} \tilde{d}_{0}-K_{1} e_{1}
$$




\subsection{StABILITY ANALYSIS}

Theorem 1: The closed loop controller scheme defined in (11), (17) and (23), respectively ensure that the error signals defined in (5), (6), and (7) are regulated as follows:

$$
e_{2}(t), \eta(t), e_{1}(t) \rightarrow 0 \text { as } t \rightarrow \infty \text {. }
$$

Proof: A non-negative Lyapunov function $V(t) \in \mathbb{R}$ is defined as:

$$
V \triangleq \frac{L_{g}}{2} e_{2}^{2}+\frac{L_{f}}{2} e_{1}^{2}+\frac{C_{f}}{2} \eta^{2}+\frac{1}{2 k_{0}} \tilde{I}_{o}^{2}+\frac{1}{2 k_{d}} \tilde{d}_{0}^{2}
$$

The time derivative of (24) is obtained as follows:

$$
\dot{V}=e_{2}\left(L_{g} \dot{e}_{2}\right)+e_{1}\left(L_{f} \dot{e}_{1}\right)+\eta\left(C_{f} \dot{\eta}\right)+k_{o}^{-1} \tilde{I}_{o} \dot{\tilde{I}}_{o}-k_{d}^{-1} \tilde{d}_{0} \dot{\hat{d}}_{0}
$$

Remark: In the voltage source inverters which are using the pulse width modulation method, the switching and sampling frequency are typically orders of magnitude higher than the fundamental frequency. Therefore, in comparison with the sampling and switching frequencies, the output current is changing very slowly, so that it can be approximated as a constant [44]. Based on this assumption the derivative of (8) can be approximated in (26):

$$
\dot{\tilde{I}}_{o}=-\dot{\hat{I}}_{o}
$$

By substitute the closed loop error dynamics of $e_{2}(t), \eta(t), e_{1}(t)$ from (11), (17), and (23) as long as the update law of $\hat{d}_{0}(t), \hat{I}_{o}(t)$ from (16) and (22), the following expression is obtained:

$$
\dot{V}=-K_{2} e_{2}^{2}+A e_{2} \eta-B \eta^{2}-K_{1} e_{1}^{2}
$$


where $A=\left(1-\frac{C_{f} K_{2}^{2}}{L_{g}}\right)$. The expression in (27) can be upper bounded and simplified by using the inequality $|\eta|\left|e_{2}\right| \leq \frac{1}{2}|\eta|^{2}+\frac{1}{2}|e|^{2}$ as:

$$
\dot{V} \leq-\left(K_{2}-\frac{A}{2}\right)\left|e_{2}\right|^{2}-\left(B-\frac{A}{2}\right)|\eta|^{2}-K_{1} e_{1}^{2}
$$

It is easy to see from (28) that $\dot{V}(t)$ is negative semi-definite if the following gain conditions are hold

$$
K_{2} \geq \frac{A}{2} \text { and } B \geq \frac{A}{2}
$$

Form (24) and (28) it is clear that $e_{2}, \eta, e_{1} \in \mathcal{L}_{\infty} \cap \mathcal{L}_{2}$ and $\tilde{d}_{0}, \tilde{I}_{o} \in \mathcal{L}_{\infty}$. Since $e_{2}, \tilde{d}_{0}, \tilde{I}_{o} \in \mathcal{L}_{\infty}$ and using Assumptions 3 and 4 as long as (4) it is easy to conclude that $I_{2}, \hat{d}_{0}, \hat{I}_{o} \in \mathcal{L}_{\infty}$. From (10) and Assumption (5) and using the fact that the time derivative of (4) is bounded we can deduce that $V_{o_{\text {ref }}} \in \mathcal{L}_{\infty}$. From (6) and $V_{o_{\text {ref }}}, \eta \in \mathcal{L}_{\infty}$ we can easily say that $V_{o} \in \mathcal{L}_{\infty}$. Since $\eta, e_{1} \in \mathcal{L}_{\infty}$ then from (16) and (22) we can see that $\dot{\hat{d}}_{o}, \dot{\hat{I}}_{o} \in \mathcal{L}_{\infty}$. Based on (9) and the above results it is clear that $\dot{e}_{2} \in \mathcal{L}_{\infty}$. Since $\eta, \hat{I}_{o}, I_{2} \in \mathcal{L}_{\infty}$ and along with Assumption 5, from (15) it is easy to say that $I_{1}^{*} \in \mathcal{L}_{\infty}$. From (17) and using the fact $e_{1}, \eta, \tilde{I}_{o}, \tilde{d}_{0} \in \mathcal{L}_{\infty}$ we can say that $\dot{\eta} \in \mathcal{L}_{\infty}$. From (23) and utilize the above results it is clear that $\dot{e}_{1} \in \mathcal{L}_{\infty}$. From (20) we can see that all the signals in the control law are bounded which is imply that $D \in \mathcal{L}_{\infty}$. Since $e_{1}, e_{2}, \eta \in \mathcal{L}_{\infty}$ and $\dot{e}_{1}, \dot{e}_{2}, \dot{\eta} \in \mathcal{L}_{\infty}$, Barbalat's Lemma [46] can be utilized to prove that $e_{2}(t), \eta(t), e_{1}(t) \rightarrow 0$ as $t \rightarrow \infty$. Thus, the control objectives are met. 


\subsection{Simulation RESUltS}

To evaluate the performance of the proposed controller, the PLECS software is used to model the instantaneous circuit dynamics of the system as well as the control scheme. The system parameters and the controller gains are listed in Table III. The steady state and the transient performances have been tested for the proposed controller scheme under different types of the local loads.

Table 0.1: Simulation Parameters

\begin{tabular}{|c|c|c|c|c|c|}
\hline Parameter & Value & Units & Gain & Value & Units \\
\hline$L_{f}$ & 10 & $m H$ & $K_{1}$ & 20 & --- \\
\hline$C_{f}$ & 50 & $\mu F$ & $K_{2}$ & 20 & --- \\
\hline$V_{d c}$ & 350 & $V$ & $K_{\eta}$ & 0.8 & --- \\
\hline$f_{s w}$ & 10 & $K H_{z}$ & $k_{d}$ & 0.1 & --- \\
\hline$L_{g}$ & 2.5 & $m H$ & $k_{0}$ & 9 & --- \\
\hline$V_{g}$ & 115 & $V_{\text {rms }}$ & & & \\
\hline \multicolumn{6}{|c|}{ Loads Parameters } \\
\hline $\begin{array}{l}\text { Resistive- } \\
\text { Inductive }\end{array}$ & \multicolumn{5}{|c|}{$\mathrm{R}=150 \Omega, \mathrm{L}=32 \mathrm{mH}$} \\
\hline Nonlinear & \multicolumn{5}{|c|}{ Single phase rectifier with $C=220 \mu F, R=250 \Omega$. } \\
\hline
\end{tabular}

In the first simulation trial a resistive-inductive ( $\mathrm{RL}$ Load) is connected to the inverter while the inverter is connected to the grid. The peak current of the desired injected current is chosen to be 4 [A]. Fig.2 and 3 demonstrate excellent steady-state tracking performance of the proposed scheme for the local load voltage $V_{o}(t)$ and the injected grid current $I_{2}(t)$. From the data of those figures we can calculate the percentage of rms steady-state error to 
be less than $1.25 \%$ for voltage and $1.5 \%$ for the current, respectively. Also Fig. 4 shows the control input $D(t)$ in this case. Figures 5 and 6 show the tracking performance of the filter inverter current $I_{1}(t)$ and the estimation performance of the output current $I_{o}(t)$, respectively.

The second simulation trial demonstrates the control scheme performance for a nonlinear local load. Figures 7 and 8 show very good tracking performance of the controller for the local load voltage and the injected grid current, respectively. Fig. 9 shows unity power factor at the grid side even with the highly distorted local load current. The total harmonic distortion (THD) for output local load voltage, grid current, and output current are 1.0\%, $2.88 \%, 86 \%$, respectively. It is seen with high distorted nonlinear local load current, the local load voltage and the injected current to the grid remain clean with very low distortion.

For the transient performance, the proposed scheme was tested by applying a $-50 \%$ step change in the amplitude of the desired grid current at the peak point to represent the worst operation case in simulation Trial 3. Fig.10 shows the current tracking responses of this test while the inverter supplying nonlinear local load.

In simulation Trial 4, the proposed controller has been tested for the seamless transition between the stand-alone and grid-connected operation modes. The system responses during the transition between the stand-alone mode and the grid-connected mode and vice versa are shown in Fig.11 and Fig.12 while the inverter supplies current to a nonlinear local load. This transition is done easily by closing and/or opening the SL/GC switch as seen in Fig.1. It is shown from Figures 11 and 12 there are no oscillations in the grid current and the local load voltage within the transition. Thus, a seamless transition in the output voltage has been achieved by the proposed controller. Based on these results, we can conclude that the 
proposed controller is working well in the two operational modes without the need to change the structure of the controller, and so the system can transfer between SA and GC modes smoothly.
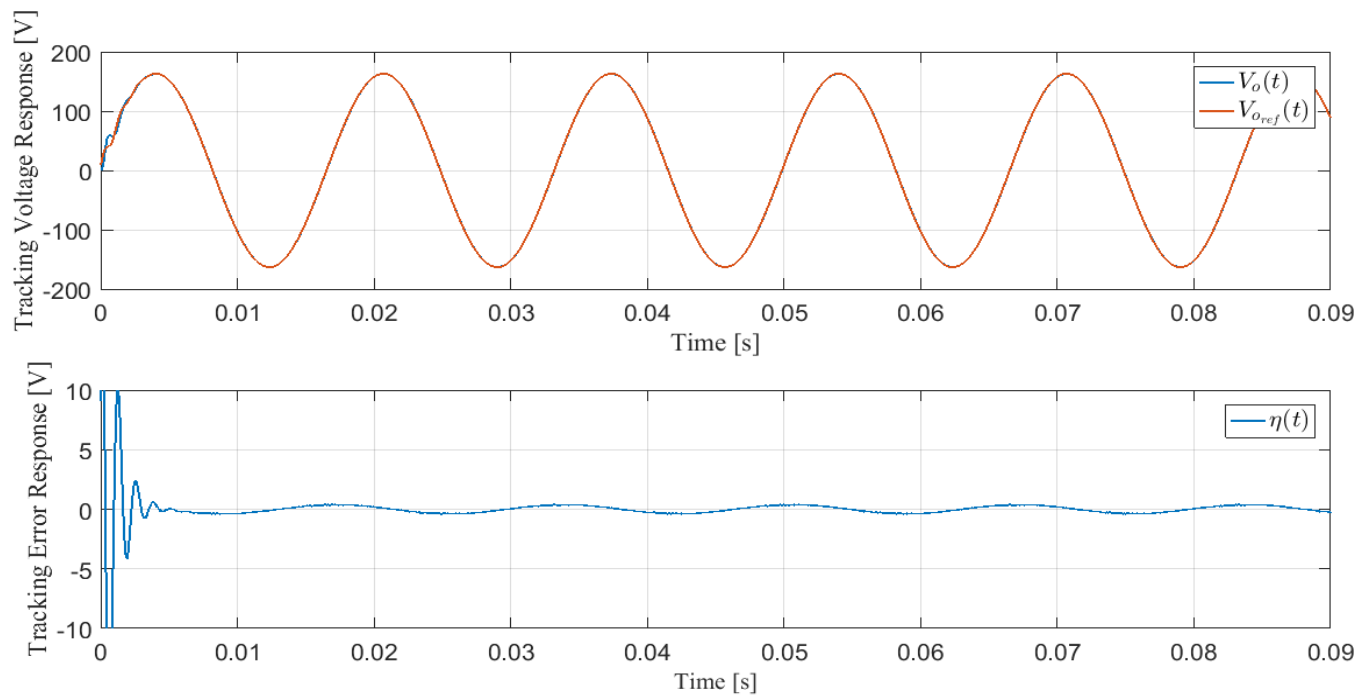

Figure 0.2 Tracking response of the output voltage for RL local load.
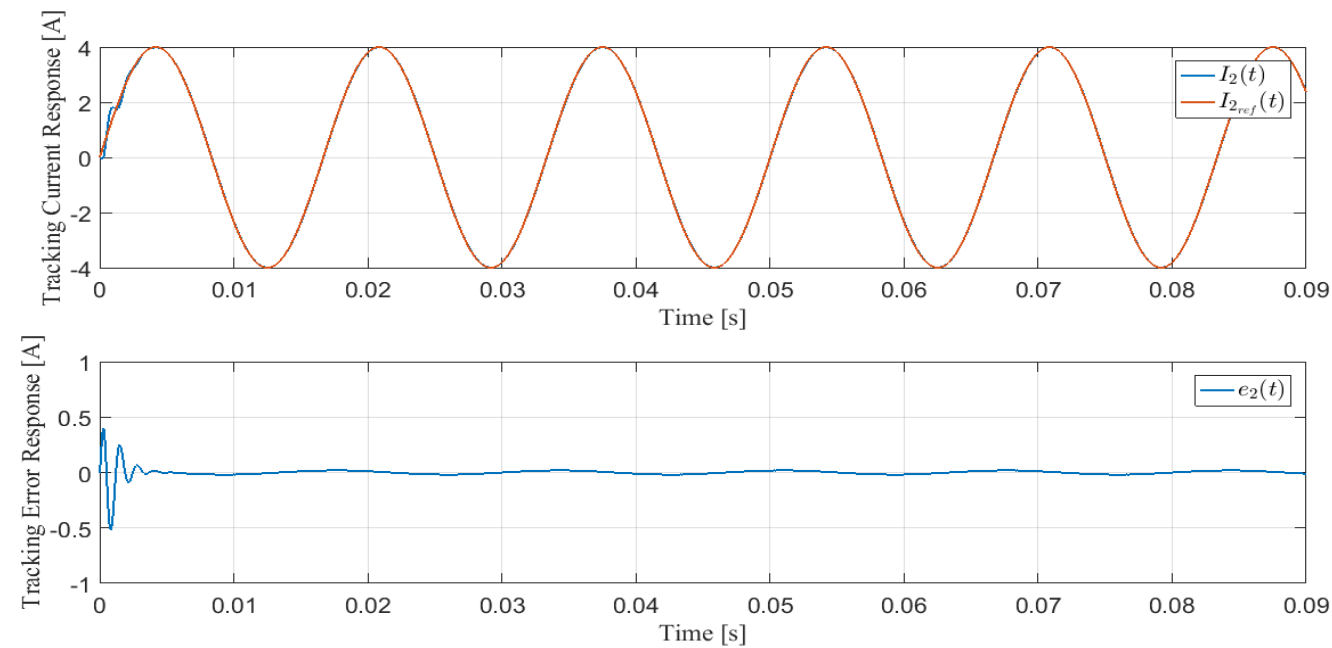

Figure 0.3 Tracking response of the grid current while the inverter supplies RL local load 


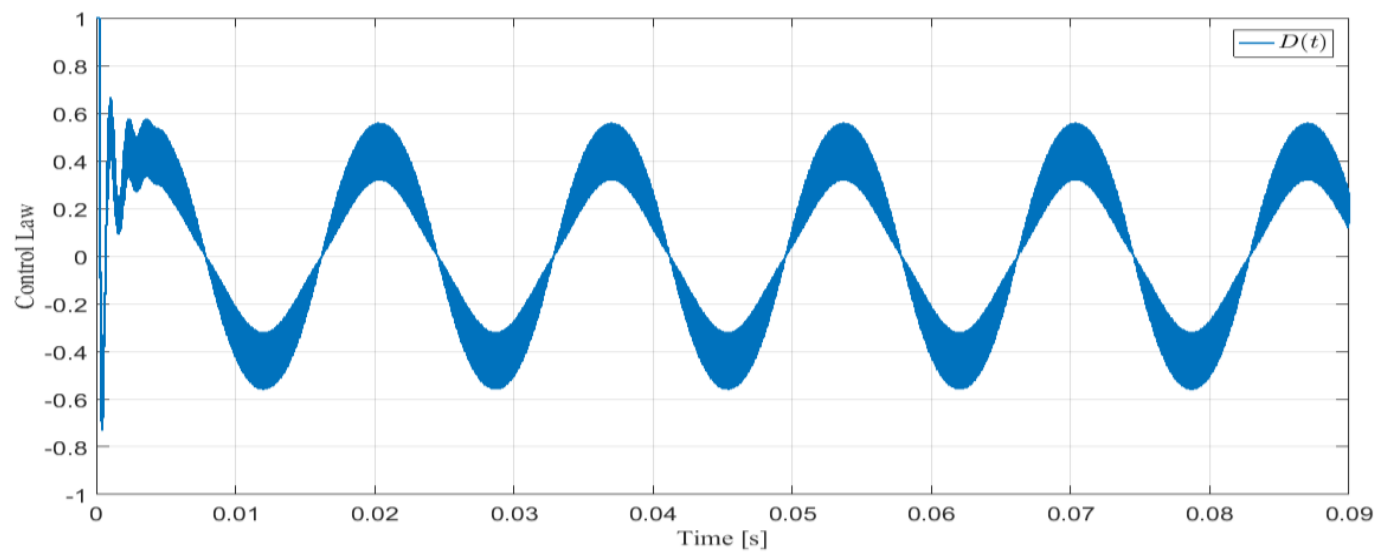

Figure 0.4 Control law under RL local load

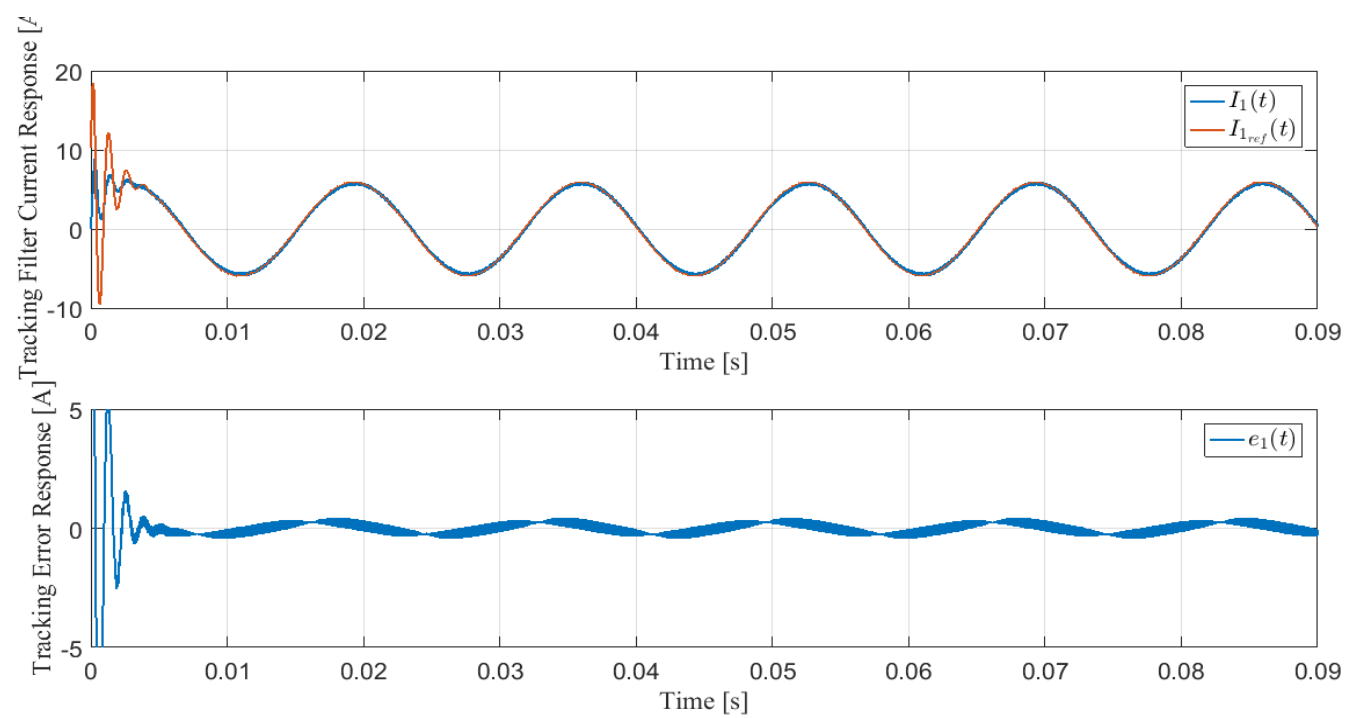

Figure 0.5 Tracking Performance of the filter inductor current under RL local load. 


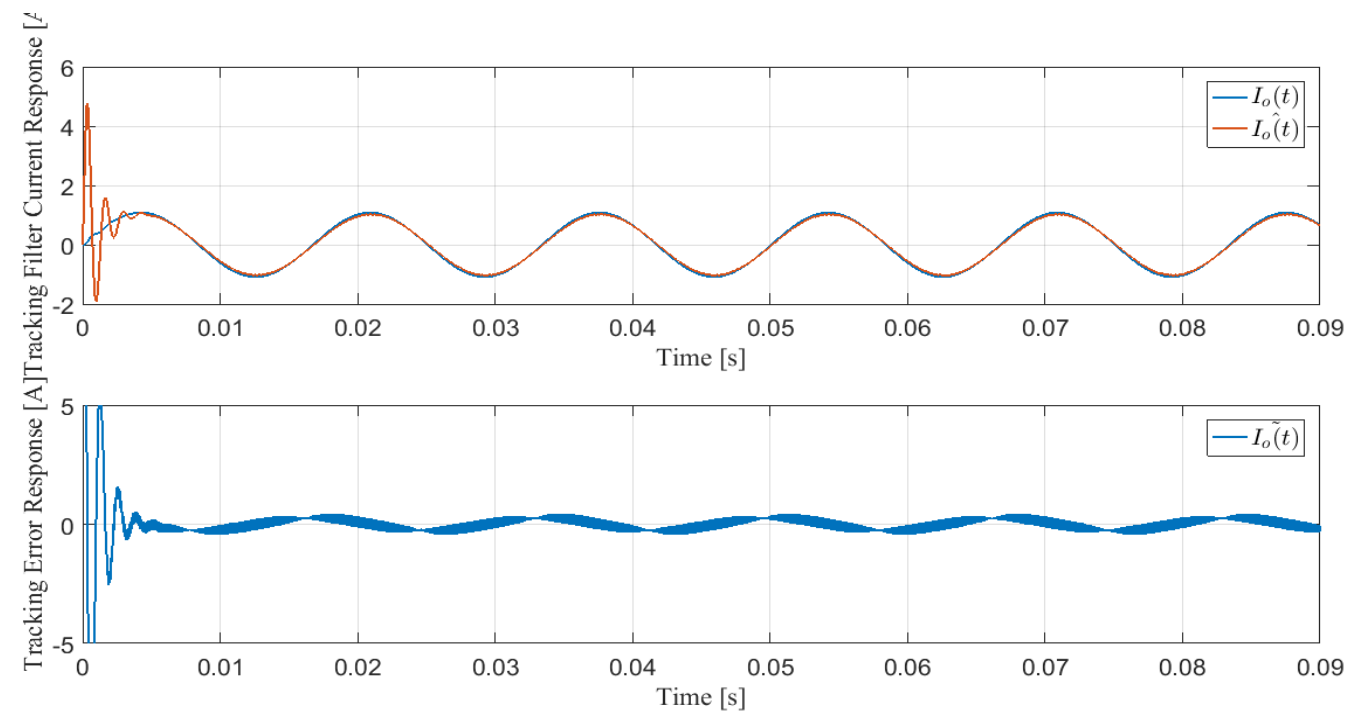

Figure 0.6 Estimation of the output current for RL local load.
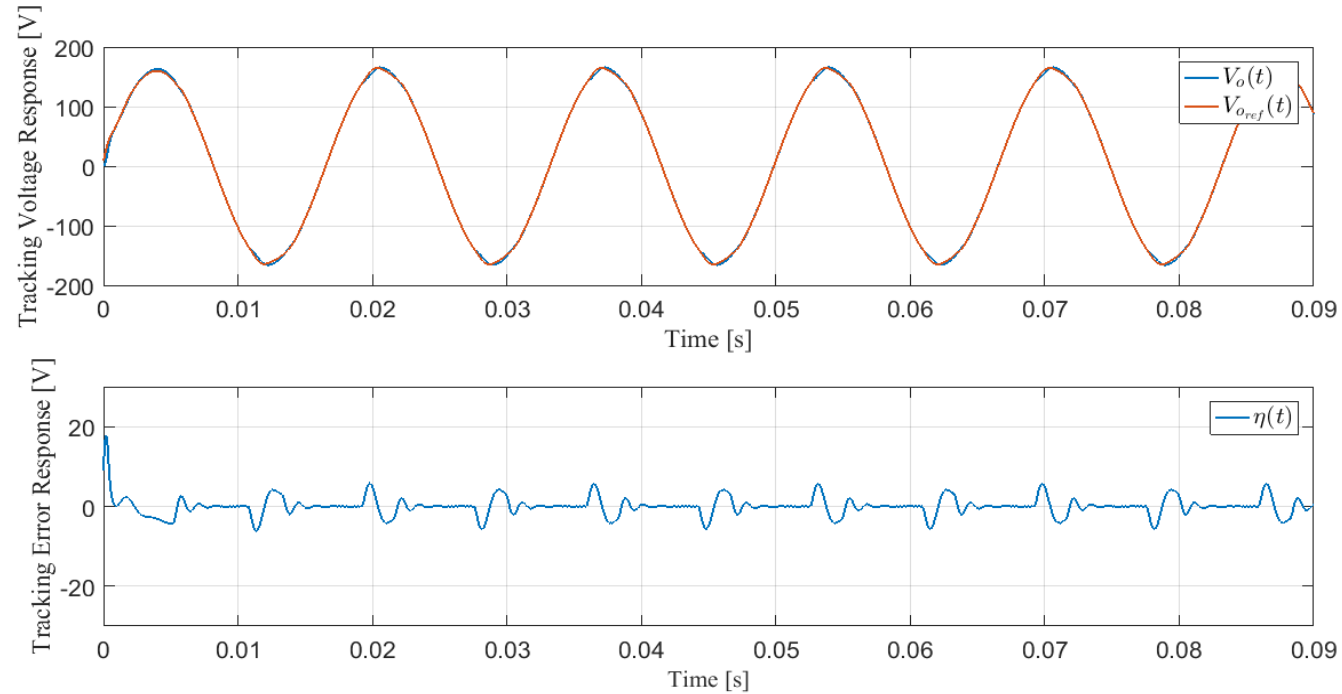

Figure 0.7 Tracking response of the output voltage for nonlinear local load. 

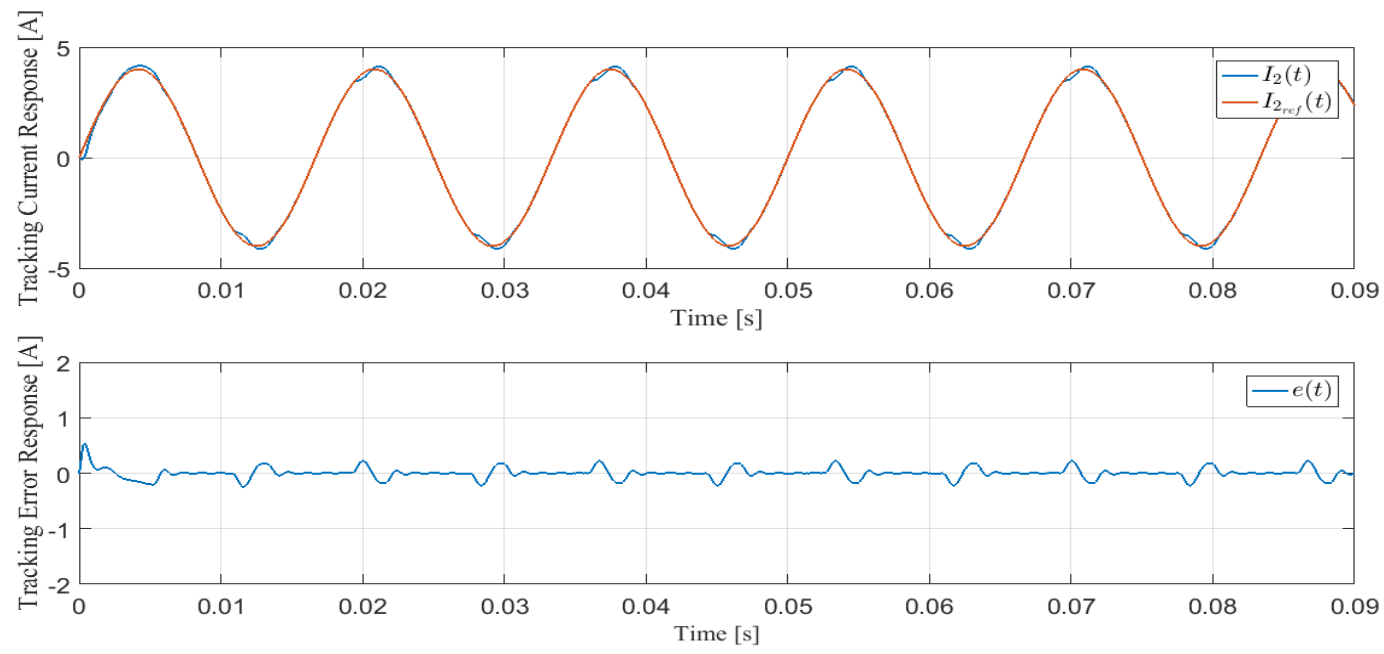

Figure 0.8 Tracking response of the grid current while the inverter supplies nonlinear local load.

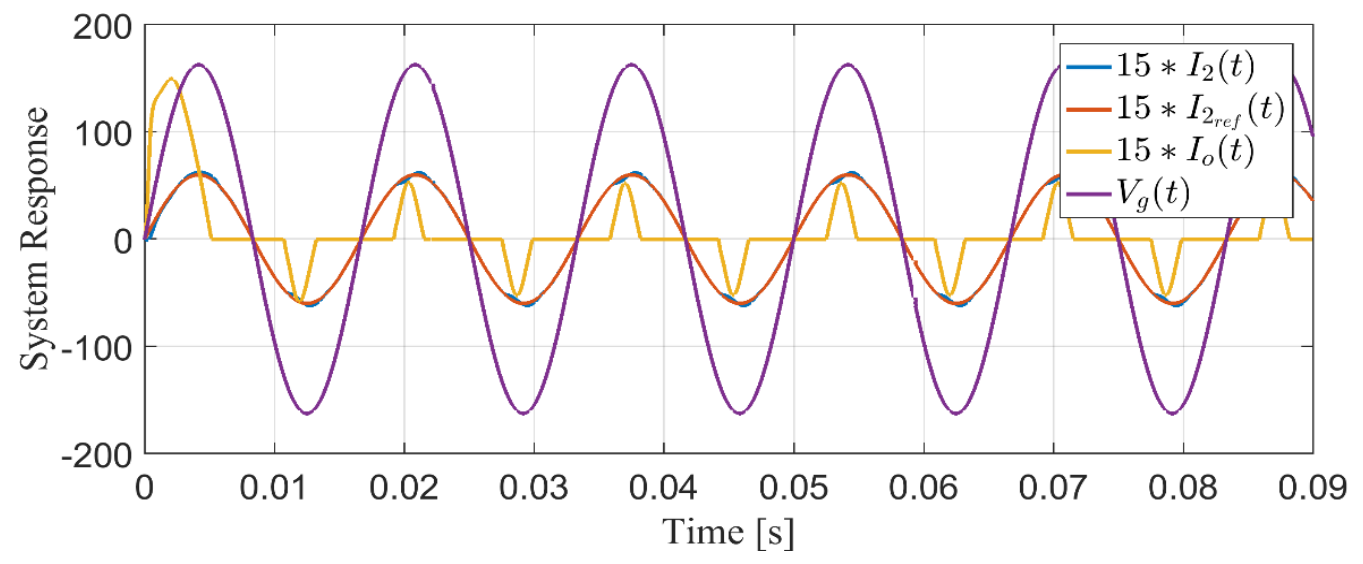

Figure 0.9 System responses of the nonlinear local load show unity power factor at grid side. 

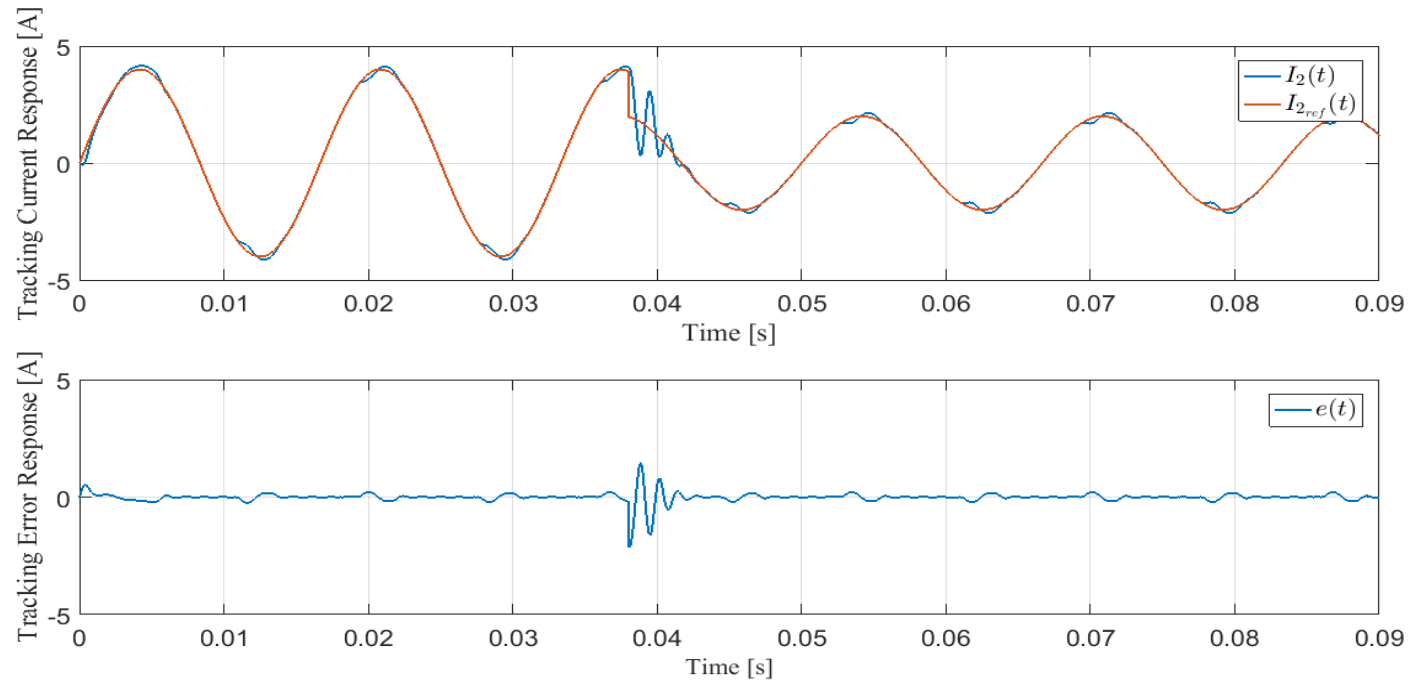

Figure 0.10 Tracking response of the grid current for $-50 \%$ step change in the reference current.
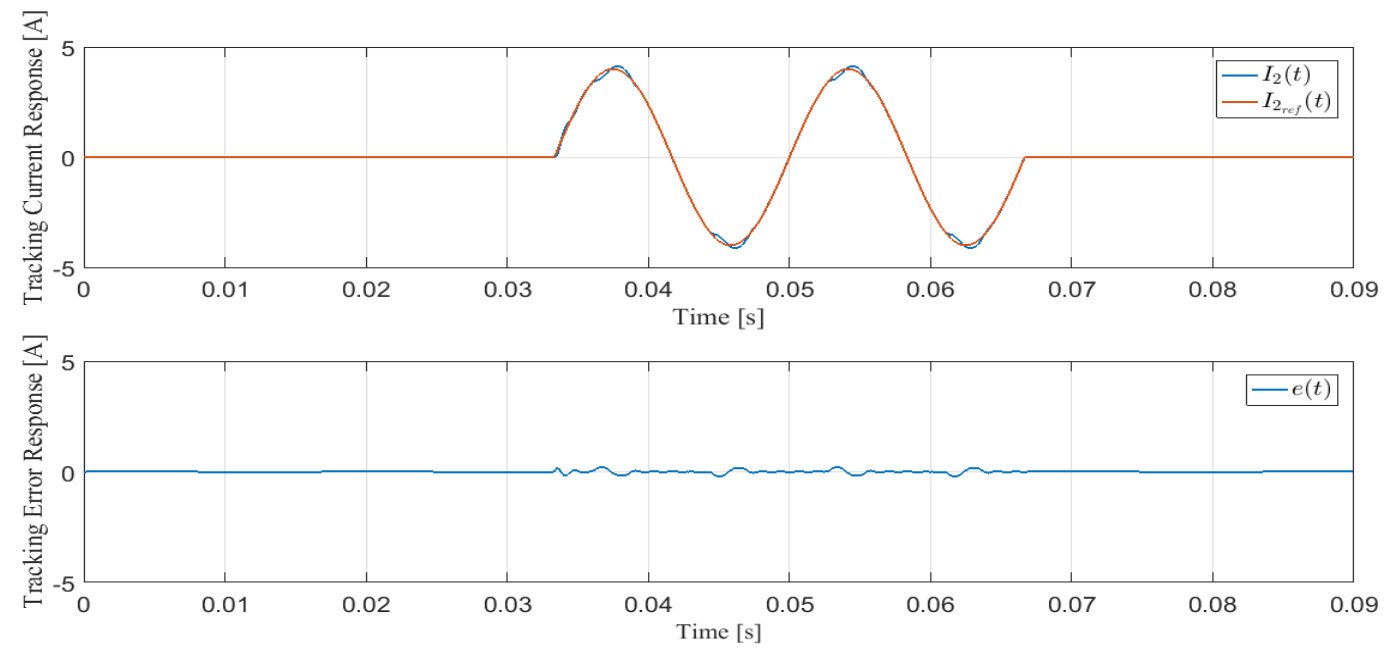

Figure 0.11 Current tracking response during seamless transfer between SA and GC modes. 

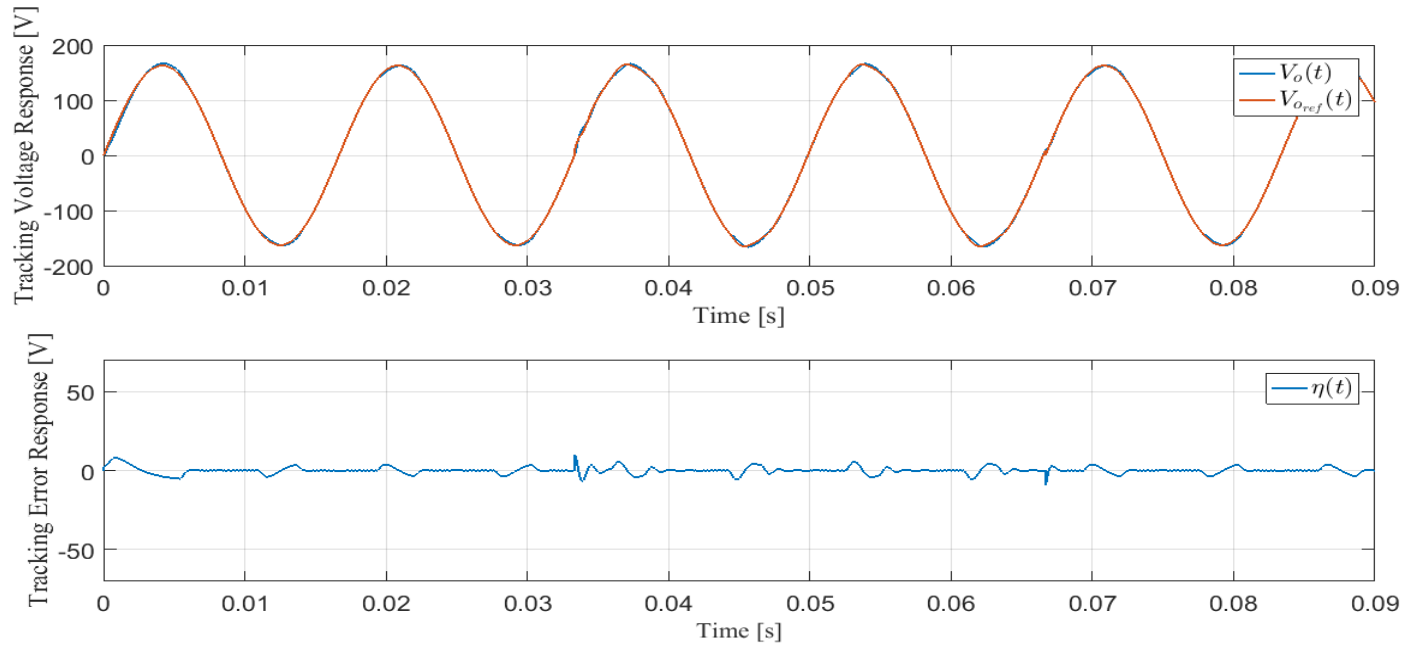

Figure 0.12 Output voltage response during seamless transfer between SA and GC modes.

\subsection{SUMMARY}

A backstepping nonlinear controller has been proposed in this chapter for a singlephase grid-connected inverter. The proposed scheme improves the quality of the local load voltage and the grid current simultaneously. Lyapunov stability analysis shows that the proposed controller scheme is stable and all signals in the closed loop system are bounded and the control objectives are met. The controller performance has been tested in the steady-state and in the transient-state via simulation. These simulation results demonstrate that the proposed controller has excellent tracking performance and is robust against changes in operating conditions. Also, a seamless transition between stand-alone and gridconnected modes has been achieved without changing the control structure. 


\section{CHAPTER 4}

\section{BACKSTEPPING CONTROL SCHEME WITH VARIBLE STRUCTURE OBSERVER}

As we can see from chapter 3 , the most common problem of the proposed backstepping controller is using the filter inductor current in the control law. This current has high frequency harmonics generated from the switching devices. Measuring such a current will add noise to the system and increase the system cost as well.

One of the contributions of this chapter is obtaining a novel second order dynamic system equation in terms of the output voltage instead of the coupled inductor-capacitor dynamics [35]. As a result, the need for a sensor for filter inductor current is removed, which will reduce the system cost while also avoiding the switching noise which is present in the actual filter inductor current. As a result of the novel modeling, the derivative of the output voltage has been introduced in this dynamic systems equation. To deal with this term, a variable structure nonlinear observer is developed.

An experimental test bed has been implemented in this chapter to further validate the proposed scheme in real-time. Efficiency have been introduced such that the scheme could be more easily implemented in an embedded platform. Moreover, comparisons with a conventional cascaded Proportional-Resonance (PR) control scheme is presented in this work to show the superiority of the proposed scheme. Through these novel advancements this approach will improve the quality of the local load voltage and simultaneously inject a clean current to the grid with the same control structure in the grid-connected mode. Moreover, the transition between stand-alone mode and grid-connected mode has been 
achieved seamlessly by using the proposed scheme without changing the controller structure.

The remainder of this chapter is organized as follows. The mathematical model of the single-phase grid-connected inverter with LC filter is presented in Section 4.1. In Section 4.2 this model is then used to develop a variable structure observer for the derivative of the output voltage. In Section 4.3 the output voltage and grid current controllers are developed and a Lyapunov stability analysis is performed for the controller- observer system. The experimental results in Section 4.4 validate the controller-observer development as well compared performance of this controller to typical control scheme. Concluding remarks are provided in Section 4.5 .

\subsection{SYSTEM MODEL}

The system in Fig.4.1 consists of the following elements: DC power source $V_{d c}$, singlephase voltage source inverter (VSI), LC filter $L_{f}, C_{f}$. The grid is emulated by an ac voltage source $V_{g}$. The grid interface inductor represented by $L_{g}$. A local load has been connected in parallel to the capacitor $C_{f}$. The mathematical differential equations for the inverter system are derived by using KVL and KCL in the state average model for $\mathrm{H}$-Bridge inverter system as follows:

$$
\begin{gathered}
L_{f} \dot{I}_{1}=V_{d c}\left(D+d_{0}\right)-V_{o} \\
C_{f} \dot{V}_{o}=I_{1}-I_{2}-I_{o} \\
L_{g} \dot{I}_{2}=V_{o}-V_{g}
\end{gathered}
$$

where $I_{1}(t), V_{o}(t), I_{2}(t) \in \mathbb{R}$ are the inductor output current, capacitor voltage which represents the output voltage, and injected current to the grid, respectively. $I_{o} \in \mathbb{R}$ is the 
current of the local load. $D(t) \in \mathbb{R}$ is duty ratio of the switching signal. $d_{0} \in \mathbb{R}$ considers the unknown disturbance representing the dead-time and voltage drop affects in the switching devices. By substituting (1) and (3) into the time derivative of (2), a second order dynamic equation for the output voltage is defined as:

$$
\ddot{V}_{o}=\frac{1}{L_{f} C_{f}}\left(V_{d c}\left(D+d_{0}\right)-V_{o}\right)+\frac{1}{L_{g} C_{f}}\left(V_{g}-V_{o}\right)+N_{0}
$$

where $N_{0} \triangleq-\frac{1}{C_{f}} \dot{I}_{o}$ is a time-varying uncertainty.

From (4) we can see that the second order dynamic equation for the output voltage doesn't have the filter inductor current. Based on (3) and (4) the proposed controller will be designed based on the backstepping approach without needs of the filter inductor current.

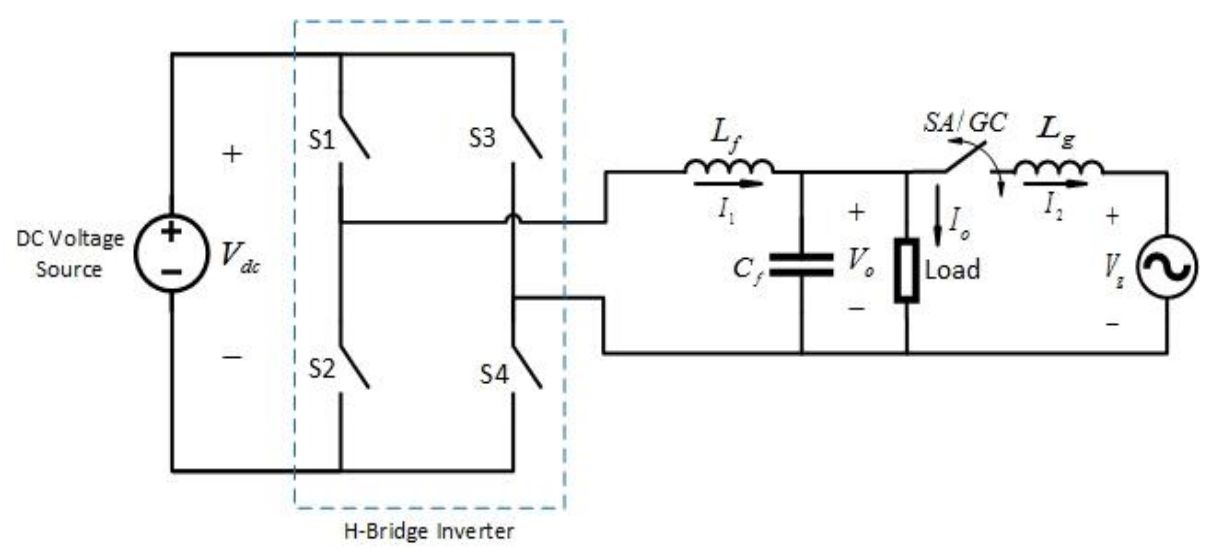

Figure 0.1 Single-phase grid-connected inverter system 


\subsection{OBSERVER DEVELOPMENT}

Prior to developing the controller, a variable structure observer for the unmeasurable $\dot{V}_{o}(t)$ is required. The subsequent development of this observer/controller is based upon the following assumptions:

Assumption 1: $L_{g}, L_{f}, C_{f}, V_{d c}$ are known, constant system parameters.

Assumption 2: The output voltage $V_{o}(t)$ is measurable.

Assumption 3: The uncertainty $N_{0}(t)$ and its derivative $\dot{N}_{0}(t)$ are bounded under normal operation, hence there exist positive numbers $\beta_{1}, \beta_{2} \in \mathbb{R}^{+}$such that $\left|N_{0}(t)\right|<$ $\beta_{1},\left|\dot{N}_{0}(t)\right|<\beta_{2}[48]$.

Assumption 4: The unknown disturbance $d_{0}$ is bounded and slowly time-varying, hence $\dot{d}_{0}(t) \approx 0$.

Assumption 5: The grid voltage is defined as $V_{g}(t)=V_{m} \sin \theta$, where $V_{m}$ is the voltage magnitude and $\theta$ is the grid phase angle. Hence, the grid voltage is bounded and differentiable.

To facilitate the observer design, the following error signals are defined to ensure that $\dot{\hat{V}}_{o}(t) \rightarrow \dot{V}_{o}(t)$ as $t \rightarrow \infty:$

$$
\begin{gathered}
\tilde{V}_{o} \triangleq V_{o}-\hat{V}_{o} \\
\dot{\tilde{V}}_{o}=\dot{V}_{o}-\dot{\hat{V}}_{o} \\
\ddot{\tilde{V}}_{o}=\ddot{V}_{o}-\ddot{\hat{V}}_{o}
\end{gathered}
$$

An additional filtered error signal $s(t) \in \mathbb{R}$ is defined as:

$$
\begin{aligned}
& s \triangleq \dot{\tilde{V}}_{o}+k_{1_{o}} \tilde{V}_{o} \\
& \dot{s}=\ddot{\tilde{V}}_{o}+k_{1_{o}} \dot{\tilde{V}}_{o}
\end{aligned}
$$


where $k_{1_{o}} \in \mathbb{R}^{+}$is filter gain. By using (4) in (7) and then substituting the result in (9), the open-loop error dynamic for $s(t)$ is defined as:

$$
\dot{s}=\frac{1}{L_{f} C_{f}}\left(V_{d c}\left(D+d_{0}\right)-V_{o}\right)+\frac{1}{L_{g} C_{f}}\left(V_{g}-V_{o}\right)+N_{0}+k_{1_{o}} \dot{\tilde{V}}_{o}-\ddot{\hat{V}}_{o}
$$

Motivated by the subsequent stability analysis and (10) an observer for $\ddot{\hat{V}}_{o}(\mathrm{t})$ can be designed as:

$$
\begin{aligned}
\ddot{\hat{V}}_{o} \triangleq & \frac{1}{L_{f} C_{f}}\left(V_{d c}\left(D+\hat{d}_{0}\right)-V_{o}\right)+\frac{1}{L_{g} C_{f}}\left(V_{g}-V_{o}\right) \\
& +k_{1_{o}} \dot{\tilde{V}}_{o}+\tilde{V}_{o}+k_{2_{o}} s+k_{3_{0}} \operatorname{sgn}\left(\tilde{V}_{o}\right)
\end{aligned}
$$

where $\operatorname{sgn}(\cdot)$ is the standard signum function, $k_{2_{0}}, k_{3_{0}}$ are positive gains and $\hat{d}_{0}(t)$ is the estimation of the unknown disturbance $d_{0}(\mathrm{t})$ with the following update law

$$
\dot{\hat{d}}_{0} \triangleq k_{d_{0}} \frac{V_{d c}}{L_{f} C_{f}} s
$$

where $k_{d_{0}}$ is a positive gain. The closed loop error dynamic for the filtered observer can be obtained by substituting (11) in (10) as

$$
\dot{s}=\frac{V_{d c}}{L_{f} C_{f}} \tilde{d}_{0}-\tilde{V}_{o}-k_{2_{o}} s+N_{0}-k_{3_{0}} \operatorname{sgn}\left(\tilde{V}_{o}\right)
$$

where $\tilde{d}_{0}(t)$ is the estimation error defined as

$$
\tilde{d}_{0} \triangleq d_{0}-\hat{d}_{0}
$$

The observer in (11) and the update law in (12) are not implementable due to their dependence on the unmeasurable signal $s(t)$. To obtain the implementable form for each one, the integration of each equation should be performed after substituting (8) into (11) and (12). For the observer dynamic, the realizable form is as follows: 


$$
\dot{\hat{V}}_{o}=p+\left(k_{1_{o}}+k_{2_{o}}\right) \tilde{V}_{o}
$$

where $p(t) \in \mathbb{R}$ is defined by

$$
\begin{gathered}
\dot{p} \triangleq \frac{1}{L_{f} C_{f}}\left(V_{d c}\left(D+\hat{d}_{0}\right)-V_{o}\right)+\frac{1}{L_{g} C_{f}}\left(V_{g}-V_{o}\right) \\
+\left(k_{1_{o}} k_{2_{o}}+1\right) \tilde{V}_{o}+k_{3_{0}} \operatorname{sgn}\left(\tilde{V}_{o}\right) .
\end{gathered}
$$

The realizable form of the disturbance update law is defined as

$$
\hat{d}_{o}=k_{d_{0}} \frac{V_{d c}}{L_{f} C_{f}}\left(\tilde{V}_{o}+k_{1_{0}} \int \tilde{V}_{o}(\sigma) d \sigma\right) .
$$

\subsection{CONTROL DEVELOPMENT}

Achieving low THD for the local load voltage $V_{o}(t)$ for a wide range of local load types is one of the control objectives for the proposed scheme. To do so, a tracking reference output voltage trajectory $V_{\text {oref }}(t)$ should be designed carefully, hence $V_{o}(t) \rightarrow$ $V_{\text {ref }}(t)$ as $t \rightarrow \infty$. The subsequent stability analysis will prove the observer objective is met; hence the voltage control objective can be modified to $\widehat{V}_{o}(t) \rightarrow V_{\text {oref }}(t)$. The other control objective is injecting low THD current to the grid by ensuring that the grid current $I_{2}(t)$ is tracking a given reference current $I_{2_{\text {ref }}}(t)$, hence $I_{2}(t) \rightarrow I_{2_{r e f}}(t)$ as $t \rightarrow \infty$. If the reference current $I_{2 \text { ref }}(t)$ is chosen as sinusoidal shape as the grid voltage, unity power factor at the grid side will be guaranteed. To do that in this work, the following reference grid current is used:

$$
I_{2_{\text {ref }}} \triangleq I_{2 p} \sin \theta
$$

where $I_{2 p}$ is the user selected peak value of the reference grid current. The grid phase angle $\theta(t)$ has been estimated based on a second order generalized integrator to create an 
orthogonal signal generator-based phase-locked loop (SOGI-OSG PLL) [47]. This is not a must, other PLL schemes could be used.

In order to facilitate the control development, the following error signals are defined:

$$
\begin{aligned}
& \eta \triangleq I_{2_{\text {ref }}}-I_{2} \\
& e=V_{o_{\text {ref }}}-\widehat{V}_{o} .
\end{aligned}
$$

After taking the time derivative of (19), pre-multiplying by $L_{g}$, substituting the system equation from (3), and using (5) and (20) we obtain the following open-loop error dynamics for $\eta(t)$ :

$$
L_{g} \dot{\eta}=L_{g} \dot{I}_{2_{\text {ref }}}-V_{o_{\text {ref }}}-\tilde{V}_{o}+e+V_{g}
$$

From (21) we can design the auxiliary control signal $V_{o_{r e f}}$ to regulate $I_{2}$ as:

$$
V_{\text {oref }} \triangleq L_{g} \dot{I}_{2 \text { ref }}+V_{g}+K_{g} \eta
$$

where $K_{g} \in \mathbb{R}^{+}$is a control gain.

Remark: The proposed control scheme has ability to work two operating modes. In the grid-connected mode, the reference grid current is $I_{2_{\text {ref }}}$ from (19) and the reference local load voltage is $V_{o_{\text {ref }}}$ from (22). In the stand-alone mode, the reference grid current is set to zero, $I_{2_{\text {ref }}}=0$. So, the reference local load voltage is $V_{o_{r e f}}=V_{g}$ as shown from (22). Under utility fault conditions when the grid voltage is not available another external signal for $V_{o_{\text {ref }}}$ can be introduced. After the grid voltage restored, we can see from (22) there is no extra efforts needed to synchronize the inverter with the grid because the reference voltage has the grid voltage information before transition from stand-alone to gridconnected mode [49].

After substituting (22) into (21), the closed loop error system for $\eta(t)$ is obtained: 


$$
L_{g} \dot{\eta}=-\tilde{V}_{o}+e-K_{g} \eta
$$

Taking the time derivative of (22), using Assumption 5, and after some simplifications, $\dot{V}_{\text {oref }}$ can be obtained for later use:

$$
\dot{V}_{o_{r e f}}=L_{g} \ddot{I}_{2_{r e f}}+K_{g} \dot{I}_{2 r e f}+\frac{K_{g}}{L_{g}}\left(V_{g}-V_{o}\right)+\omega V_{m} \cos \theta
$$

where $\omega(t)=\dot{\theta}(t)$ is the grid angular frequency. Substituting the observer dynamic from (15) into the time derivative of (20) and using (24), the open loop error dynamics for $e(t)$ can be obtained in the following:

$$
\dot{e}=L_{g} \ddot{I}_{2_{r e f}}+K_{g} \dot{I}_{2_{r e f}}+\frac{K_{g}}{L_{g}}\left(V_{g}-V_{o}\right)+\omega V_{m} \cos \theta-p-\left(k_{1_{o}}+k_{2_{o}}\right) \tilde{V}_{o} .
$$

To facilitate the control development an auxiliary tracking controller $p_{d}(t) \in \mathbb{R}$ is defined for $p(t)$ from (25) as:

$$
p_{d} \triangleq L_{g} \ddot{I}_{2 r e f}+K_{g} \dot{I}_{2 r e f}+\frac{K_{g}}{L_{g}}\left(V_{g}-V_{o}\right)+\omega V_{m} \cos \theta+K_{1 c} e-\left(k_{1_{o}}+k_{2_{o}}\right) \tilde{V}_{o}
$$

where $K_{1 c} \in \mathbb{R}^{+}$is a control gain. The closed loop error dynamic for $e(t)$ can be obtained after substituting (26) into (25)

$$
\dot{e}=-K_{1 c} e+\tilde{p}
$$

where $\tilde{p}(t)$ is the tracking error for the auxiliary controller $p_{d}(t)$ defined as:

$$
\tilde{p} \triangleq p_{d}-p
$$

Substituting (16) and the time derivative of (26) into the time derivative of (28) and after some simplifications, the open loop error dynamic of the auxiliary controller $\tilde{p}(t)$ is obtained as:

$$
\dot{\tilde{p}}=L_{g} \dddot{I}_{2_{r e f}}+K_{g} \ddot{I}_{2 r e f}+\frac{K_{g}}{L_{g}}\left(\omega V_{m} \cos \theta-\dot{\hat{V}}_{o}\right)-\bar{K} \dot{\tilde{V}}_{o}
$$




$$
\begin{gathered}
-\frac{1}{L_{g} C_{f}}\left(V_{g}-V_{o}\right)-K_{1 c}^{2} e+K_{1 c} \tilde{p}-\overline{\bar{K}} \tilde{V}_{o}-k_{3_{0}} \operatorname{sgn}\left(\tilde{V}_{o}\right) \\
-\omega^{2} V_{m} \sin \theta-\frac{1}{L_{f} C_{f}}\left(V_{d c}\left(D+\hat{d}_{0}\right)-V_{o}\right)
\end{gathered}
$$

where $\bar{K}=k_{1_{o}}+k_{2_{o}}+\frac{K_{g}}{L_{g}}, \quad \overline{\bar{K}}=\left(k_{1_{o}} k_{2_{o}}+1\right)$.

Based on this open loop error dynamic and motivated by the subsequent stability analysis, the control law is designed as:

$$
\begin{gathered}
D \triangleq \frac{L_{f} C_{f}}{V_{d c}}\left[L_{g} \dddot{I}_{2_{r e f}}+K_{g} \ddot{I}_{2 r e f}-\frac{1}{L_{g} C_{f}}\left(V_{g}-V_{o}\right)+\frac{K_{g}}{L_{g}}\left(\omega V_{m} \cos \theta-\dot{\hat{V}}_{o}\right)\right. \\
-\omega^{2} V_{m} \sin \theta-K_{1 c}^{2} e-\overline{\bar{K}} \tilde{V}_{o}-k_{3_{0}} \operatorname{sgn}\left(\tilde{V}_{o}\right)+\frac{V_{o}}{L_{f} C_{f}} \\
\left.+K_{2 c} \tilde{p}+e\right]-\hat{d}_{0}
\end{gathered}
$$

where $K_{2 c} \in \mathbb{R}^{+}$is a control gain. The closed loop error dynamic for $\tilde{p}(t)$ can be obtained by substituting (30) into (29)

$$
\dot{\tilde{p}}=-\bar{K} \dot{\tilde{V}}_{o}-K \tilde{p}-e
$$

where $K \triangleq K_{2 c}-K_{1 c}$.

\subsection{STABILITY ANALYSIS}

Theorem 1: The closed loop observer/controller scheme defined in (13), (23), (27) and (31) ensures that $\widehat{V}_{o} \rightarrow V_{o}, \dot{\hat{V}}_{o} \rightarrow \dot{V}_{o}, \hat{V}_{o} \rightarrow V_{o_{\text {ref }}}$ and $I_{2} \rightarrow I_{2 \text { ref }}$ as $t \rightarrow \infty$. Proof: A non-negative Lyapunov function $V(t) \in \mathbb{R}$ is defined as: 


$$
V \triangleq \frac{1}{2} \tilde{V}_{o}^{2}+\frac{1}{2} s^{2}+\frac{1}{2 k_{d_{0}}} \tilde{d}_{0}^{2}+\frac{L_{g}}{2} \eta^{2}+\frac{1}{2} e^{2}+\frac{1}{2} \tilde{p}^{2}+P_{0}
$$

where $P_{0}(t) \in \mathbb{R}$ is a term defined as follows to make sure that $V(t)$ is positive definite:

$$
P_{0} \triangleq \beta_{0}-\int_{t_{0}}^{t} L_{0}(\sigma) d \sigma
$$

where $L_{0}(t) \in \mathbb{R}$ has been defined as

$$
L_{0} \triangleq s\left(N_{0}-k_{3_{0}} \operatorname{sgn}\left(\tilde{V}_{o}\right)\right)
$$

From (33) we can note that $P_{0}(t)$ is positive if there exist a positive number $\beta_{0}$ such that $\beta_{0}>\int_{t_{0}}^{t} L_{0}(\sigma) d \sigma$. The full proof of this is given in [50]. The time derivative of (32) is

$$
\dot{V}=\tilde{V}_{o} \dot{\tilde{V}}_{o}+s \dot{s}+\frac{1}{k_{d_{0}}} \tilde{d}_{0} \dot{\tilde{d}}_{0}+L_{g} \eta \dot{\eta}+e \dot{e}+\tilde{p} \tilde{\tilde{p}}+\dot{P}_{0}
$$

After substituting $\dot{\tilde{V}}_{o}$ from (8), the error dynamics from (13), (23), (27), and (29), and the disturbance update law from (12) as well as the time derivative of (33) into (35), the following expression can be obtained

$$
\dot{V}=-K_{g} \eta^{2}-\eta \widetilde{V}_{o}+\eta e-K_{1 c} e^{2}-K \tilde{p}^{2}-k_{1_{o}} \tilde{V}_{o}^{2}-k_{2_{o}} s^{2}-\bar{K} s \tilde{p}+k_{1 o} \bar{K} \tilde{V}_{o} \tilde{p}
$$

The inequality $|\eta||e| \leq \frac{1}{2}|\eta|^{2}+\frac{1}{2}|e|^{2}$ could be used to upper bounded the above expression as follows:

$$
\begin{gathered}
\dot{V} \leq-\left(K_{g}-\frac{1}{2}\right)|\eta|^{2}-\left(K_{1 c}-\frac{1}{2}\right)|e|^{2}-\frac{K}{2}|\tilde{p}|^{2}-\frac{k_{2 o}}{2}|s|^{2} \\
-\frac{k_{1 o}}{2}\left|\tilde{V}_{o}\right|^{2}+|s|\left(\bar{K}|\tilde{p}|-\frac{k_{2 o}}{2}|s|\right)+|\tilde{p}|\left(k_{1 o} \bar{K}\left|\tilde{V}_{o}\right|-\frac{K}{2}|\tilde{p}|\right)+\left|\widetilde{V}_{o}\right|\left(|\eta|-\frac{k_{10}}{2}\left|\tilde{V}_{o}\right|\right) .
\end{gathered}
$$

Nonlinear damping technique can be used to simplify the last three bracketed terms in (37), and then $\dot{V}$ can be further upper bounded as: 


$$
\begin{gathered}
\dot{V} \leq-\left(K_{g}-\frac{1}{k_{1_{o}}}-\frac{1}{2}\right)|\eta|^{2}-\left(\frac{K}{2}-2 \bar{K}^{2} / k_{2_{o}}\right)|\tilde{p}|^{2} \\
-\left(K_{1_{c}}-\frac{1}{2}\right)|e|^{2}-\left(\frac{k_{1 o}}{2}-\frac{2\left(k_{1_{o}} \bar{K}\right)^{2}}{K}\right)\left|\tilde{V}_{o}\right|^{2}-\frac{k_{2_{o}}}{2}|s|^{2} .
\end{gathered}
$$

If the following gain conditions are met, it is easy to see from (38) that $\dot{V}(t)$ is negative semi-definite

$$
\begin{gathered}
K_{g}>\frac{1}{2}+\frac{1}{k_{1_{o}}}, \quad K_{1_{c}}>1 / 2 \\
K>4 \bar{K}^{2} / k_{2_{o}}, \quad K>4 \bar{K}^{2} / k_{1_{o}}, \quad K_{2_{c}}>K_{1_{c}} .
\end{gathered}
$$

From (32) and (38) it is clear that $e, \eta, \tilde{p}, \tilde{V}_{o}, s \in \mathcal{L}_{\infty} \cap \mathcal{L}_{2}$ and $\tilde{d}_{0}, \tilde{d} \in \mathcal{L}_{\infty}$. Since $e, \tilde{d}, \tilde{p} \in$ $\mathcal{L}_{\infty}$, we can see from (27) that $\dot{e} \in \mathcal{L}_{\infty}$. From (12) it is easy to see that $\dot{\hat{d}}_{0} \in \mathcal{L}_{\infty}$. Also, $\hat{d}_{0} \in \mathcal{L}_{\infty}$ based on (14) and Assumption 4. From (8) and since $\tilde{V}_{o}, s \in \mathcal{L}_{\infty}$ we can see that $\dot{\tilde{V}}_{o} \in \mathcal{L}_{\infty}$. Based on (31) and $e, \tilde{p}, \dot{\tilde{V}}_{o} \in \mathcal{L}_{\infty}$ we conclude that $\dot{\tilde{p}} \in \mathcal{L}_{\infty}$. From (18) we can say that the reference current and its first, second, and third derivative are bounded. By using the above fact, $\eta \in \mathcal{L}_{\infty}$ and Assumption 5, from (22) it is easy to say that $V_{o_{r e f}} \in \mathcal{L}_{\infty}$. From (20) and using $e, V_{o_{\text {ref }}} \in \mathcal{L}_{\infty}$ one can see that $\widehat{V}_{o} \in \mathcal{L}_{\infty}$. Since $\widehat{V}_{o}, \tilde{V}_{o} \in \mathcal{L}_{\infty}$ then from (5) we can see that $V_{o} \in \mathcal{L}_{\infty}$. We can conclude that $\dot{\eta} \in \mathcal{L}_{\infty}$ since all signals in (23) are bounded. Form (24) and along with above results we can see that $\dot{V}_{o_{\text {ref }}} \in \mathcal{L}_{\infty}$. From the derivative of (20) and by using $\dot{V}_{\text {ref }^{\prime}}, \dot{e} \in \mathcal{L}_{\infty}$ it is easy to say that $\dot{\hat{V}}_{o} \in \mathcal{L}_{\infty}$. Form (13) and Assumption 3 one can conclude that $\dot{s} \in \mathcal{L}_{\infty}$. From above results we can see that all signals in (30) are bounded, so we can say that $D \in \mathcal{L}_{\infty}$. Therefore, all signals in the closed loop dynamics are bounded. Since $e, \eta, \tilde{p}, \tilde{V}_{o}, s \in \mathcal{L}_{\infty} \cap \mathcal{L}_{2}$ and $\dot{e}, \dot{\eta}, \dot{\tilde{p}}, \dot{\tilde{V}}_{o}, \dot{s} \in \mathcal{L}_{\infty}$ Barbalat's 
Lemma [46] can be utilized to prove that $e, \eta, \tilde{p}, \tilde{V}_{o}, s \rightarrow 0$ as $t \rightarrow \infty$. Thus, completing the proof of Theorem 1.

\subsection{EXPERIMENTAL RESULTS}

Real-time implementation has been developed as shown in Fig.2 to evaluate the performance of the proposed controller-observer scheme. A doubler-rectifier circuit has been used to generate the dc bus voltage as input of the H-bridge single-phase inverter. A two-quadrant programmable AC source BK- PRECISION 9803 750VA was used to emulate the utility grid at fundamental frequency $60 \mathrm{~Hz}$. A power resistor $\left(R_{p}\right)$ has been placed in parallel with this AC source and sized such that its power draw exceeds the power which is sourced by the inverter (as set by the value of $I_{2 p}$ ). This is done to ensure that current is never sent to the AC source. The NI CompactRIO 9063 with LabVIEW software has been used to implement the proposed algorithm and to execute it in real-time by the onboard Virtex-5 LX50 FPGA. The control diagram of the proposed scheme is shown in Fig.3. Table I summarizes the system parameters and the observer/controller gains. Both the steady state and transient performances of the proposed scheme have been tested while the inverter injecting current to the grid and supporting different types of the local loads in the same time. For comparison purposes, the well-known Proportional-Resonance (PR) controller has been designed based on [51]. Because we have voltage and current control objectives, the cascaded approach is used to design two control loops. The outer current control loop generates the reference voltage for the inner voltage loop as shown in Fig.4. 
The grid voltage is fed forward to the output of the current controller to make sure the inverter is synchronized to the grid.

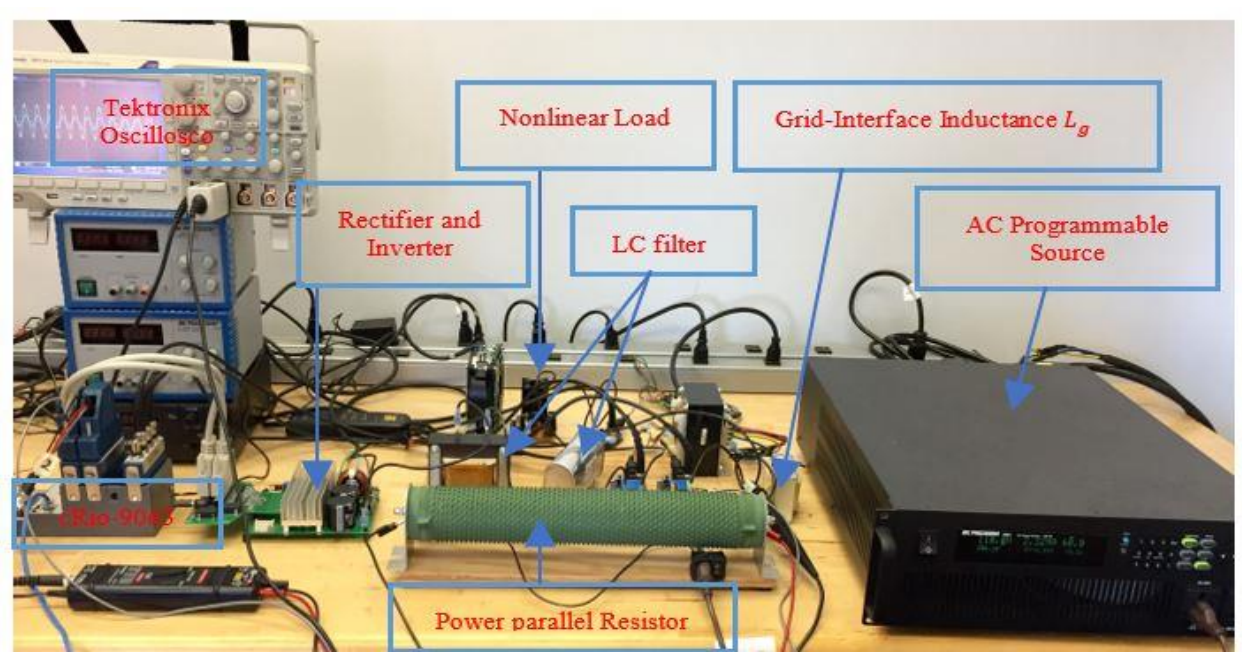

Figure 0.2 Experimental setup of the grid-connected inverter

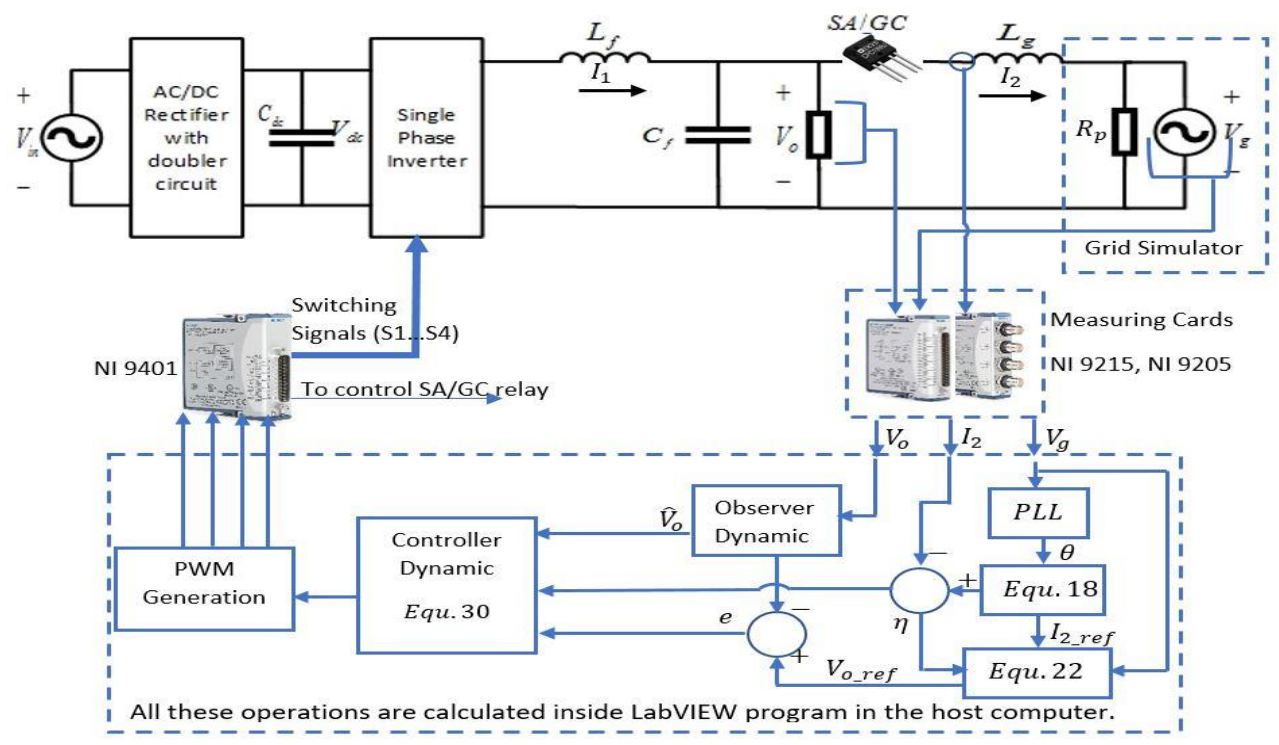

Figure 0.3 Block diagram for the proposed controller/observer scheme 


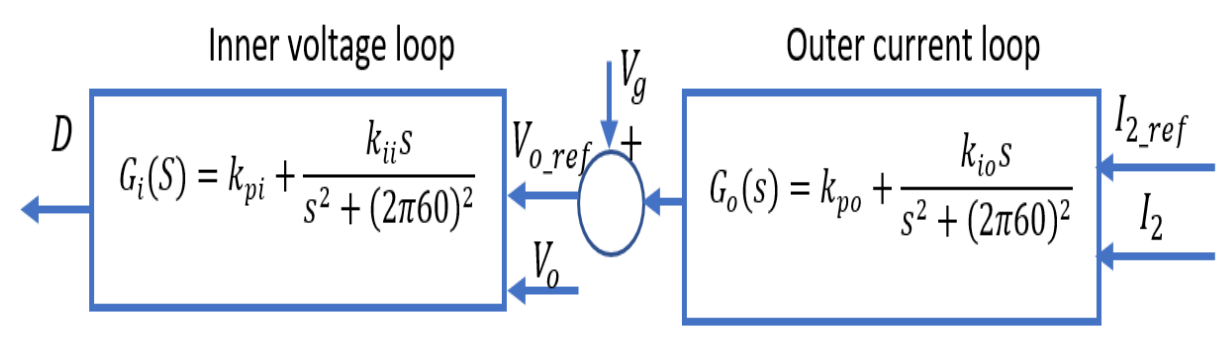

Figure 0.4 Block diagram for cascaded PR control scheme

$\underline{\text { Table 0.1 System and Control Parameters }}$

\begin{tabular}{|c|c|c|c|c|c|c|}
\hline$L_{f}[m H]$ & $C_{f}[\mu F]$ & $L_{g}[m H]$ & $V_{d c}[V]$ & $f_{s w}\left[K H_{z}\right]$ & $\boldsymbol{f}_{s}\left[\boldsymbol{K} \boldsymbol{H}_{\boldsymbol{Z}}\right]$ & $V_{g}[V]$ \\
\hline 10 & 50 & 2.5 & 300 & 16 & 32 & 110 \\
\hline $\boldsymbol{k}_{\mathbf{1}_{o}}$ & $k_{2}$ & $\boldsymbol{k}_{3_{o}}$ & $K_{1 c}$ & $K_{2 c}$ & $K_{g}$ & $\boldsymbol{k}_{d_{0}}$ \\
\hline 1000 & 10000 & 200000 & 7000 & 20000 & 22 & $1 * 10^{-10}$ \\
\hline \multirow{2}{*}{$\begin{array}{l}\text { Local } \\
\text { loads }\end{array}$} & \multirow{2}{*}{$\mathbf{R L}$} & $R[\Omega]$ & $L[m H]$ & \multirow{2}{*}{ NL } & $R[\Omega]$ & $C[\mu F]$ \\
\hline & & 150 & 32 & & 250 & 220 \\
\hline \multicolumn{7}{|c|}{ Cascaded PR Control Scheme gains for RL load (Before tuning) } \\
\hline \multirow{2}{*}{ Inner } & $k_{i i}$ & $\boldsymbol{k}_{p i}$ & \multirow{2}{*}{ Outer } & $\boldsymbol{k}_{i o}$ & $\boldsymbol{k}_{p o}$ & \\
\hline & 50 & 7 & & 10 & 1 & \\
\hline
\end{tabular}

In the first experiment a resistive-inductive local load (RL Load) is connected to the inverter while the inverter is connected to the grid. The amount of the injected power to the grid is determined by choosing the value of the reference current. In this test, the peak value of the reference current in (18) is selected to be 3[A]. Fig.5 shows the steady-state tracking performance of the local load voltage $V_{o}(t)$ for both the proposed scheme and the cascaded PR controller. The injected grid current $I_{2}(t)$ response for both schemes are shown in Fig.6. The gains for the cascaded PR control scheme in this test are shown in Table I. The percentage of the rms steady-state errors and the THD for both controllers are summarized in Table II. Total harmonic distortion in the voltages and currents are used to assess the power quality for the inverter systems that are connected to the utility grid. The 
maximum THD for the voltage and current for such systems is 5\% [4]. Tektronix DPO 3014 digital oscilloscope was used in this work to measure the THD for both the voltage and current. The tracking performance of the proposed observer is shown in Fig.7. The control input $D(t)$ in this case is shown in Fig.8.

In the second experiment, the performance of the proposed scheme and the cascaded PR control scheme has been tested when the nonlinear local load (NL load) is connected to the inverter while the inverter is connected to the grid. The cascaded PR control scheme failed to deal with the changing of the local load type which leads the system to be unstable. To complete the comparison, a gain tuning process has been completed to return the system to the stability region and to minimize the steady-state error. The new gains are obtained to be $k_{i i}=200, k_{p i}=17, k_{i o}=100, k_{p o}=1$. Fig. 9 and 10 show the tracking performance of the proposed and the cascaded PR controllers for the local load voltage and the injected grid current, respectively. Table II shows that the proposed scheme outperforms cascaded PR scheme in the percentage rms steady-state error as well as the THD for both the local load voltage and the injected current to the grid while the inverter supplies power to the nonlinear local load and to the grid simultaneously. Fig. 11 demonstrates unity power factor at the grid side even with the highly distorted local load current. From the previous results, it is clear that the proposed scheme is injecting very clean current to the grid while supporting the local load with low THD voltage. Also, the proposed scheme is robust for the changing local load type, but the cascaded PR controller needs tuning the gains for each local load type.

To test the transient performance for the proposed scheme and the cascaded PR scheme, a step change in the amplitude of the reference grid current from 2 [A] to 3 [A] is applied 
during grid-connected mode. This step is applied at the peak point in order to illustrate the worst operation while connecting nonlinear local load to the inverter. Fig. 12 shows that the grid current immediately follows the reference grid current for the proposed scheme. For the cascaded PR controller, the current needs around 16 cycles to follow its reference.
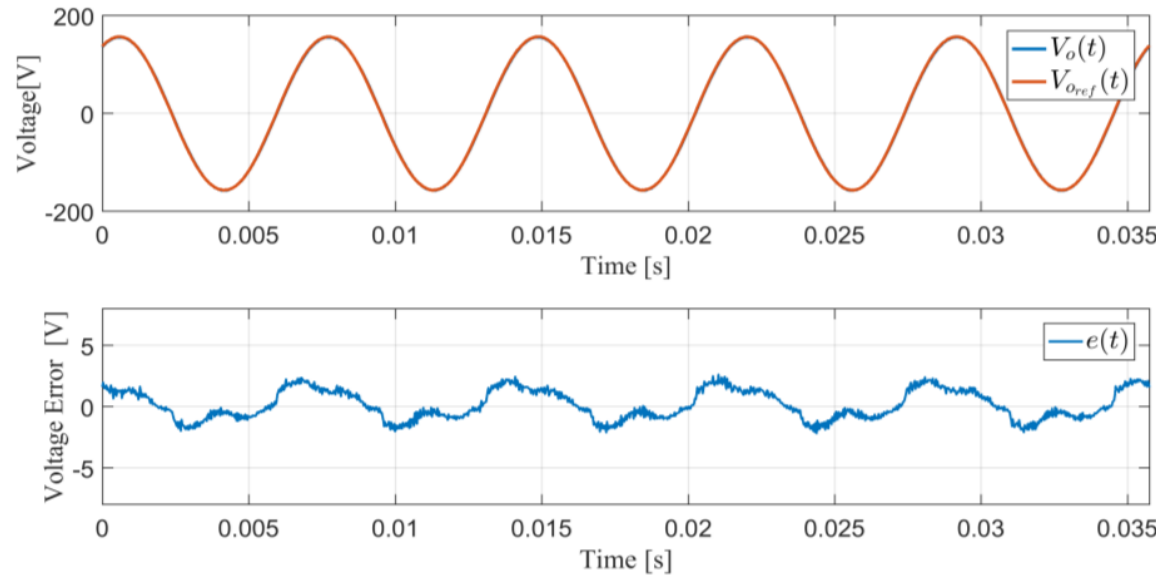

(a)
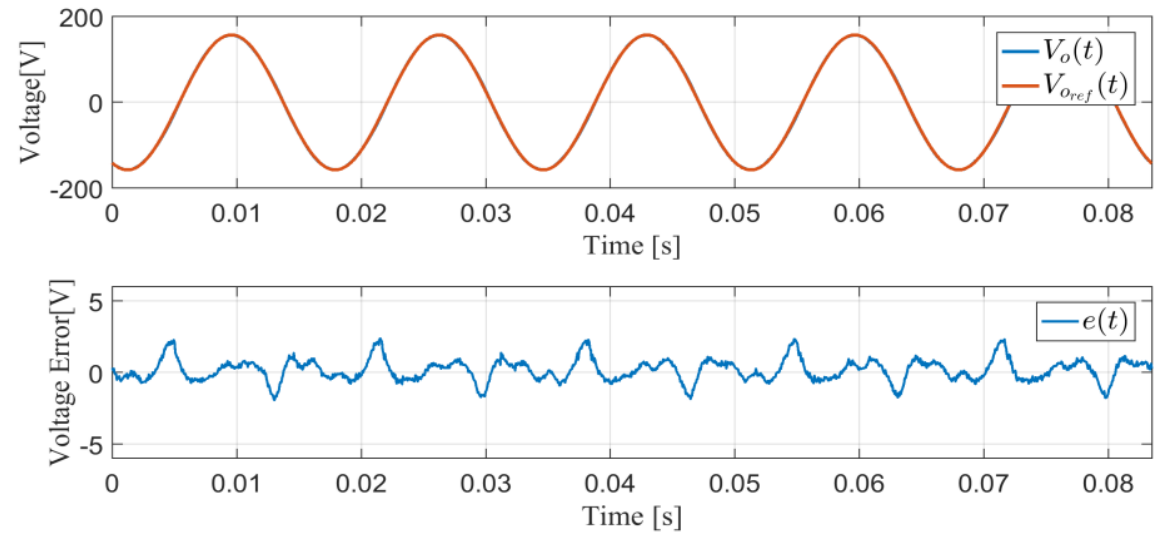

(b)

Figure 0.5 Tracking response of the output voltage for RL local load.

(a) Proposed Scheme (b) Cascaded PR controller. 

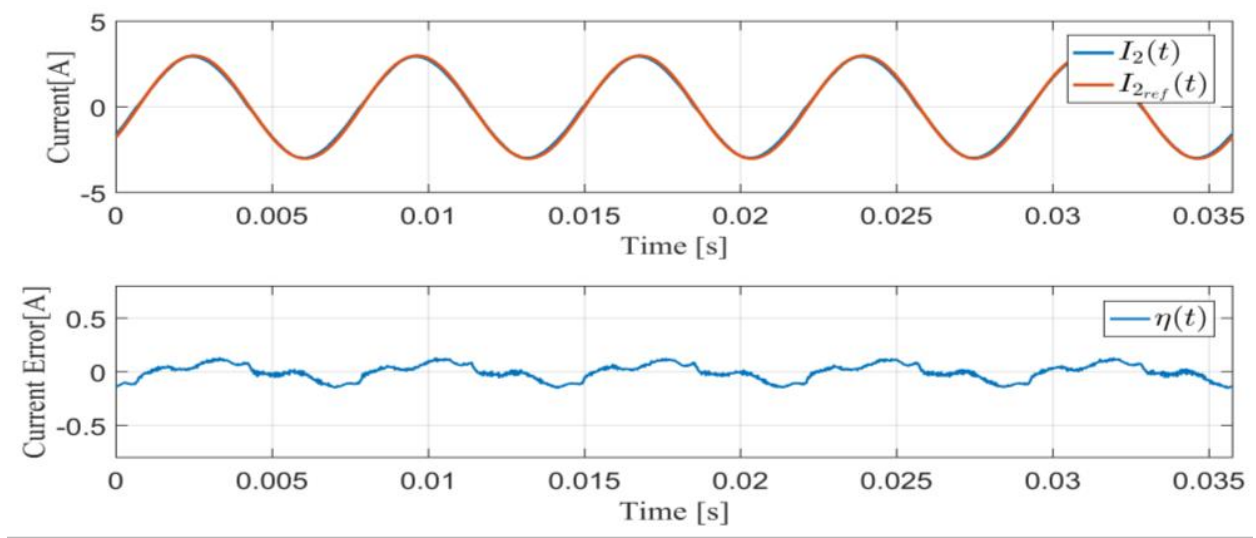

(a)
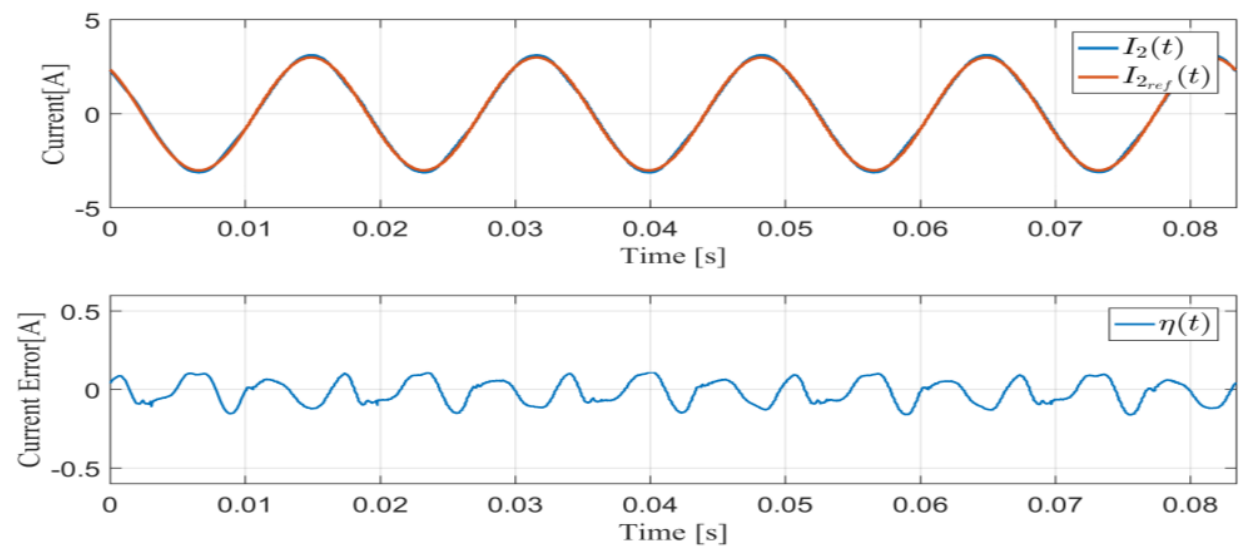

(b)

Figure 0.6 Tracking response of the grid current under RL local load.

(a) Proposed Scheme (b) Cascaded PR controller.
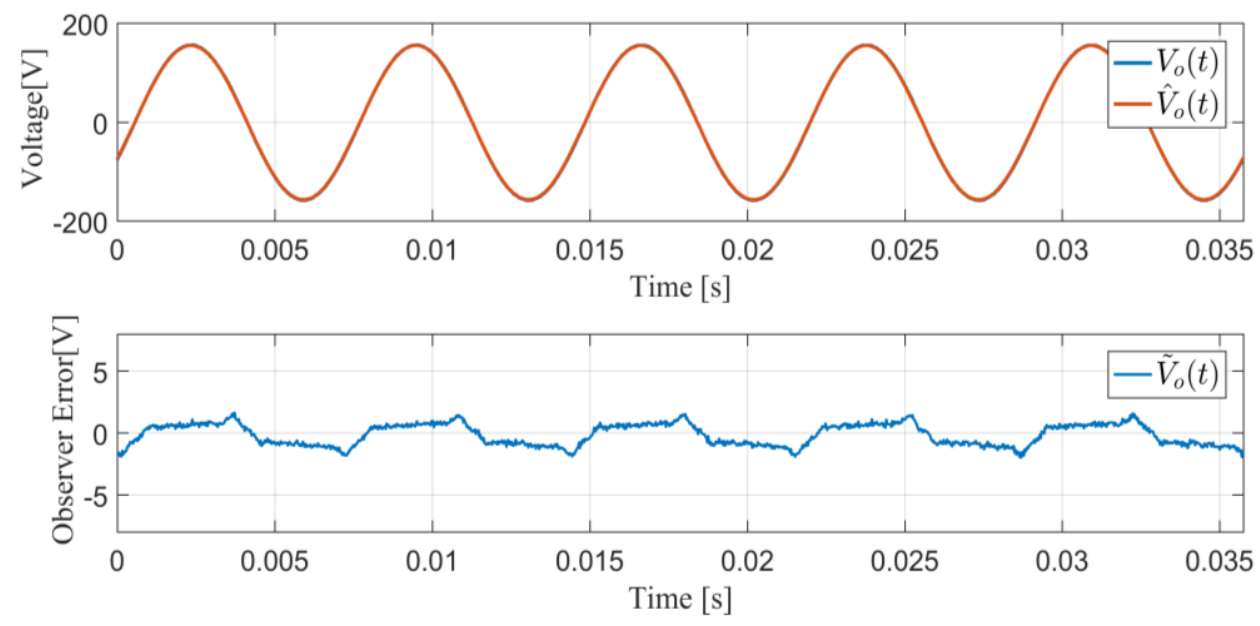

Figure 0.7 Observer tracking performance under RL local load. 


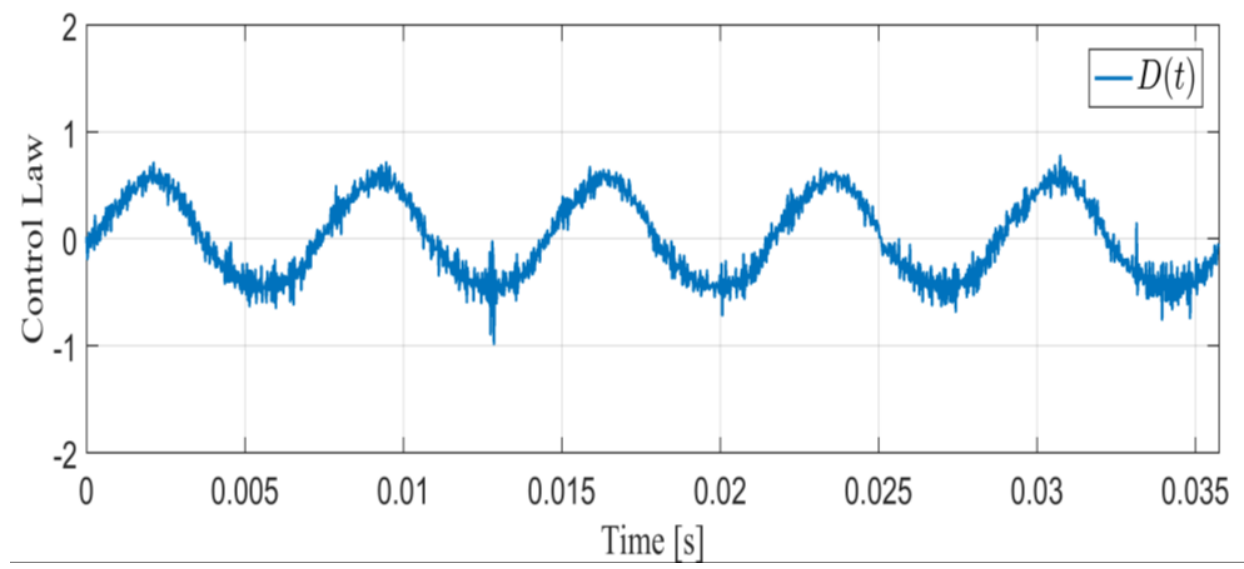

Figure 0.8 Control law for the proposed scheme under RL local load.
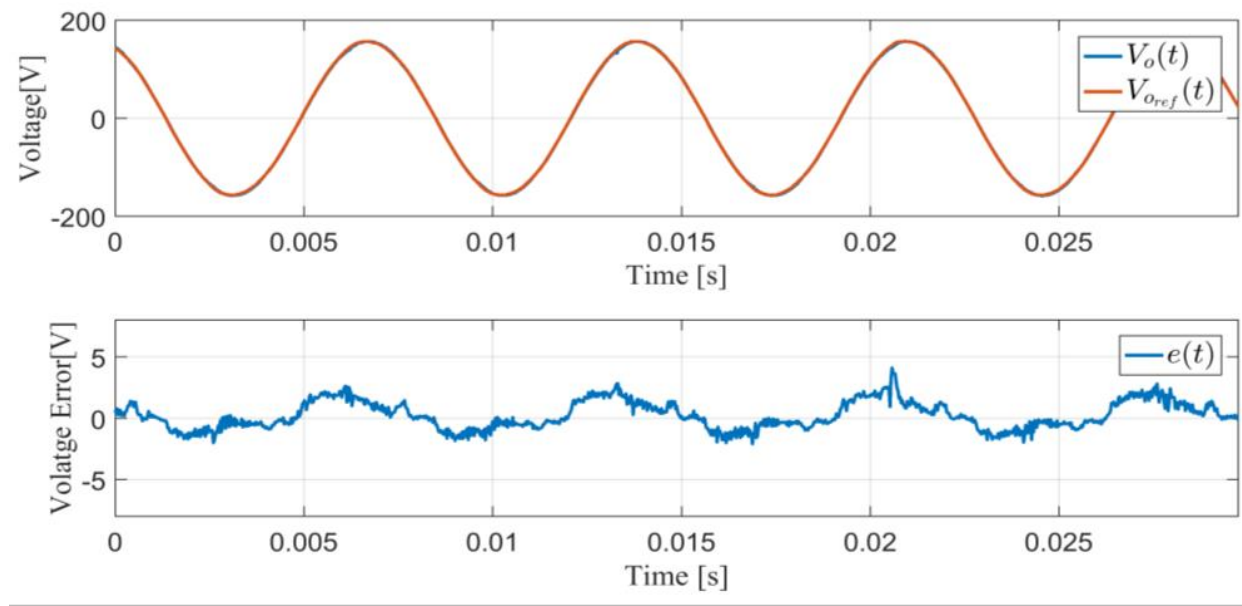

(a)
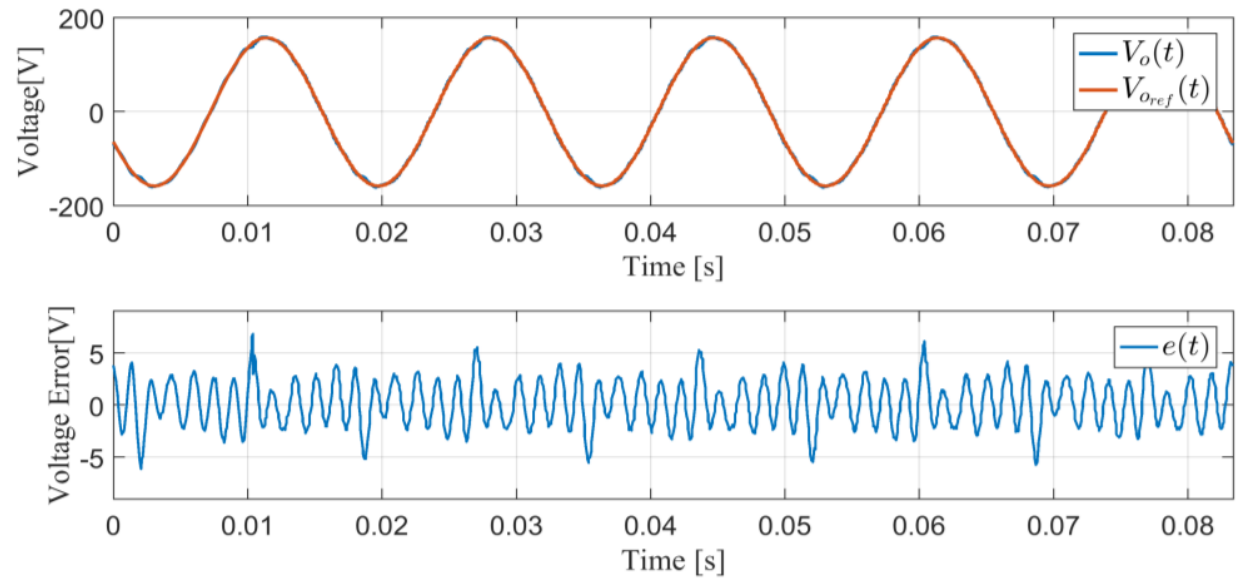

(b)

Figure 0.9 Tracking response of the output voltage for NL local load.

(a) Proposed Scheme (b) Cascaded PR controller (required gains tuning). 

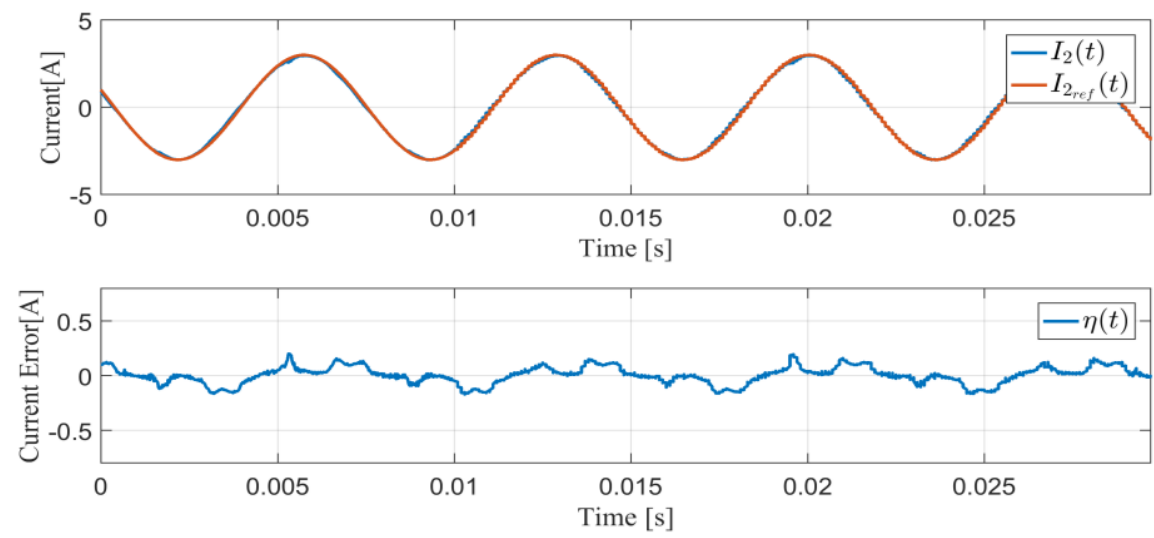

(a)
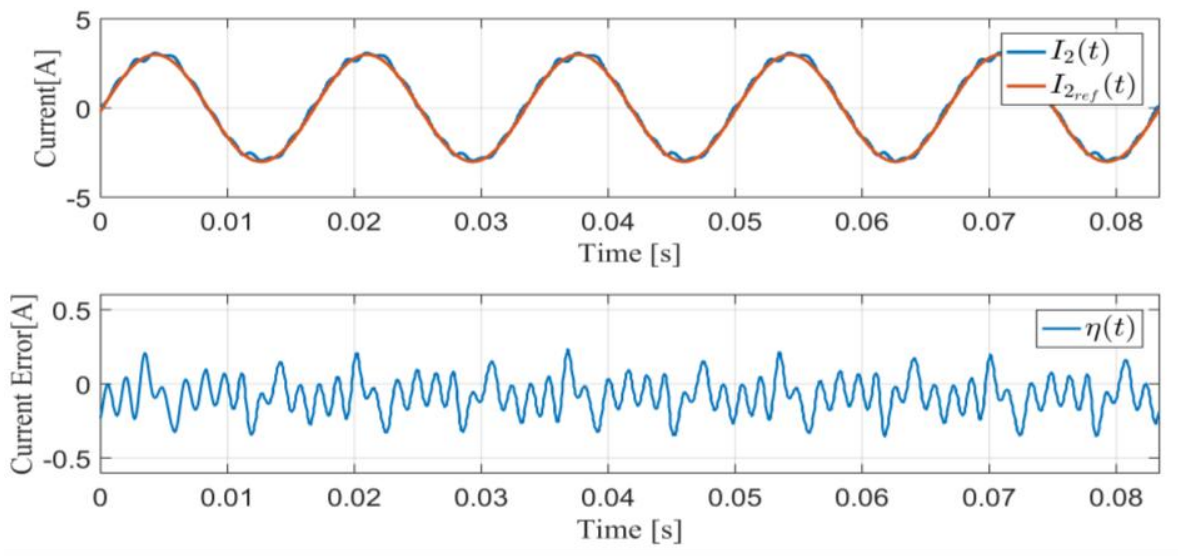

(b)

Figure 0.10 Tracking response of the grid current under NL local load.

(a) Proposed Scheme (b) Cascaded PR controller (required gains tuning).

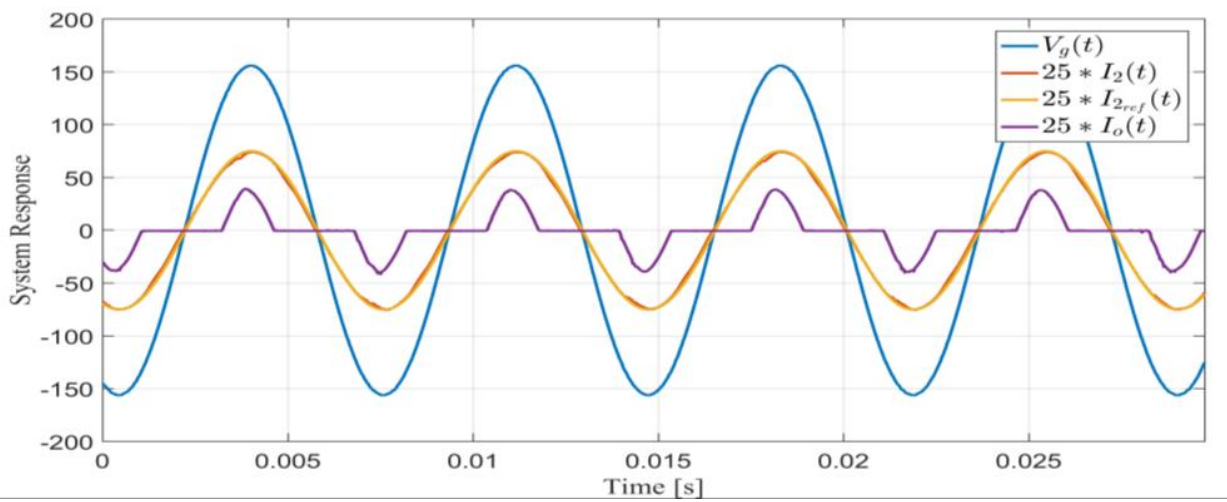

Figure 0.11 System response to show unity power factor under NL local load. 

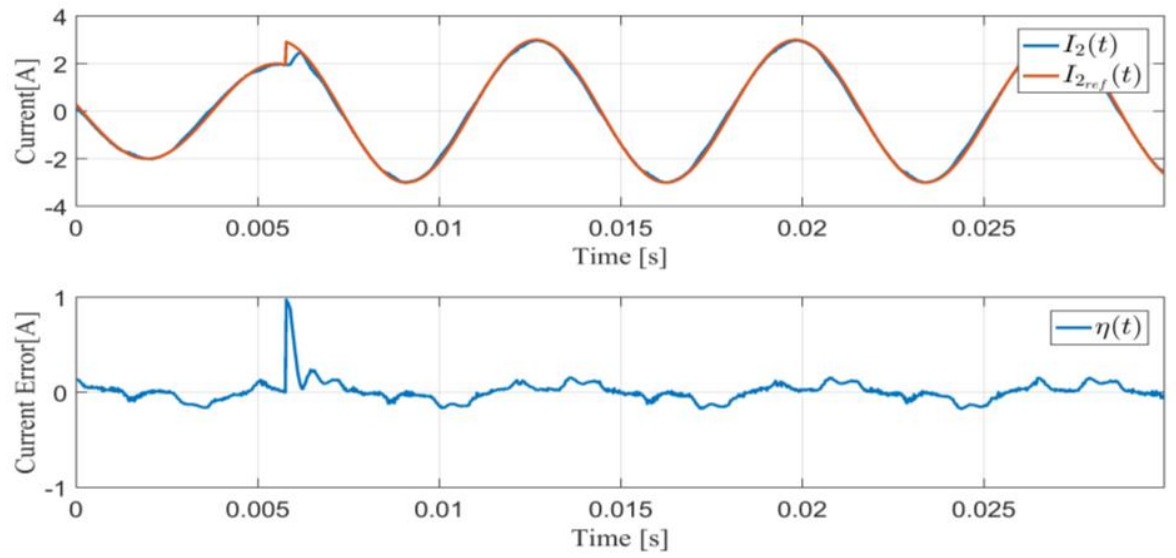

(a)
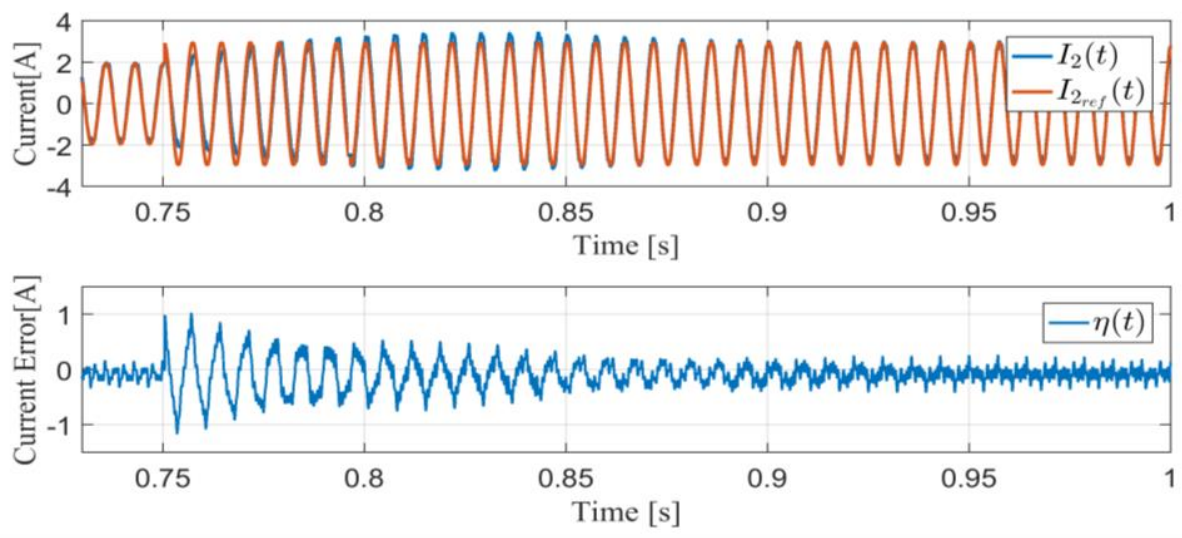

(b)

Figure 0.12 Tracking response of the grid current during reference current step change under NL load (a) Proposed Scheme (b) Cascaded PR controller (required gains tuning).
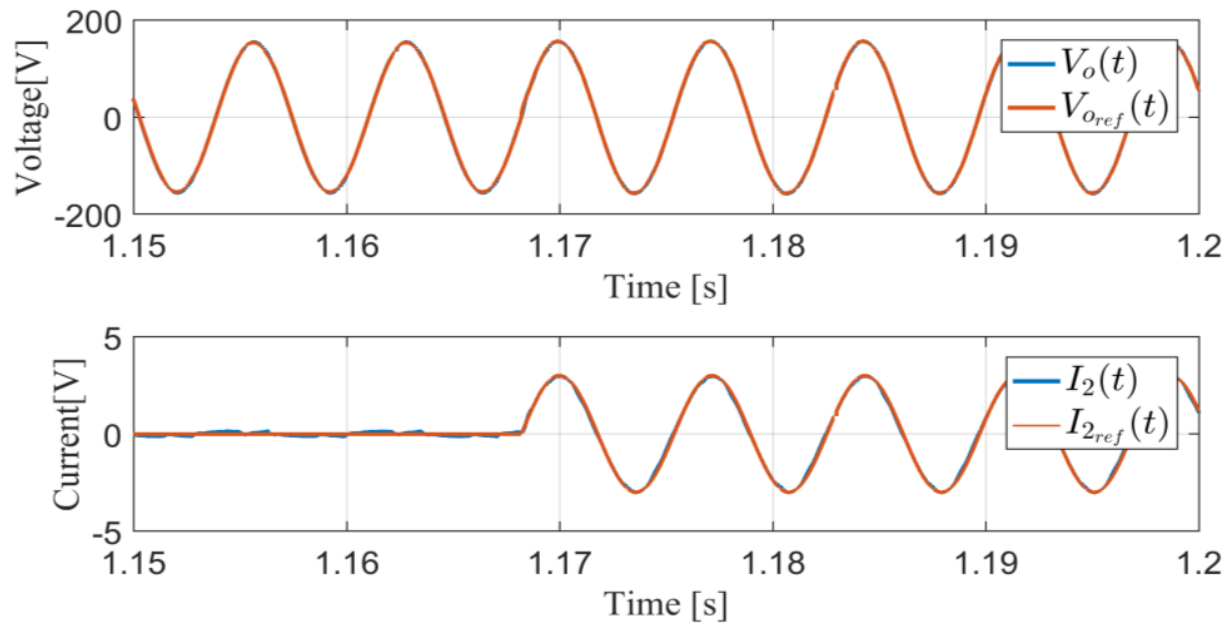

(a) 

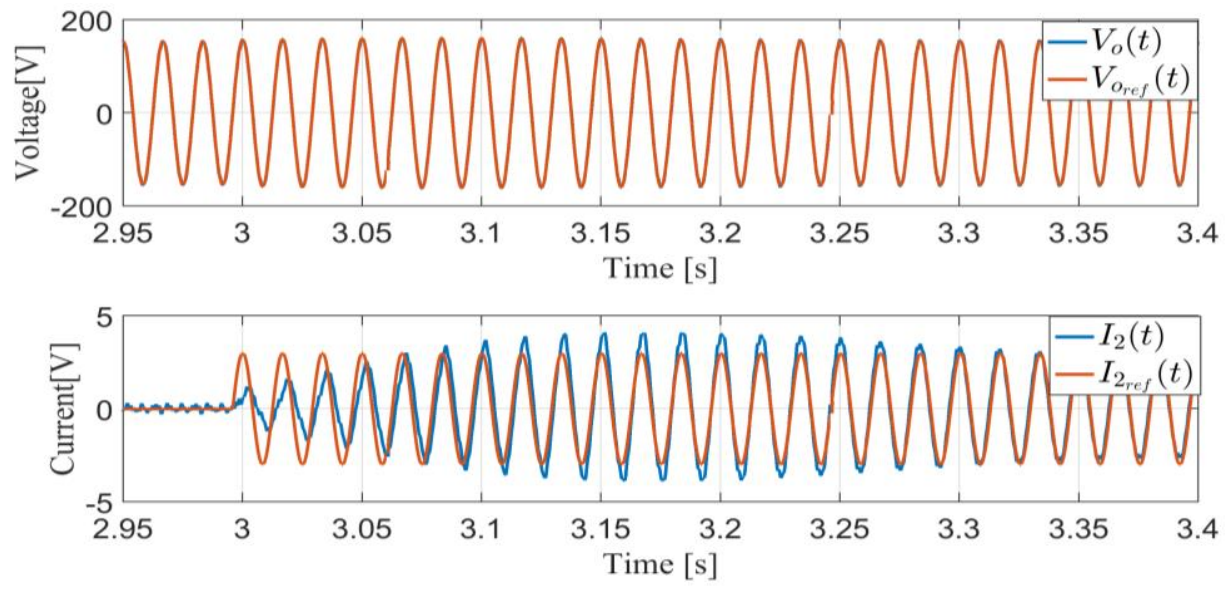

(b)

Figure 0.13 Voltage and current tracking response during transfer from SA mode to GC mode. (a) Proposed Scheme (b) Cascaded PR controller (required gains tuning).
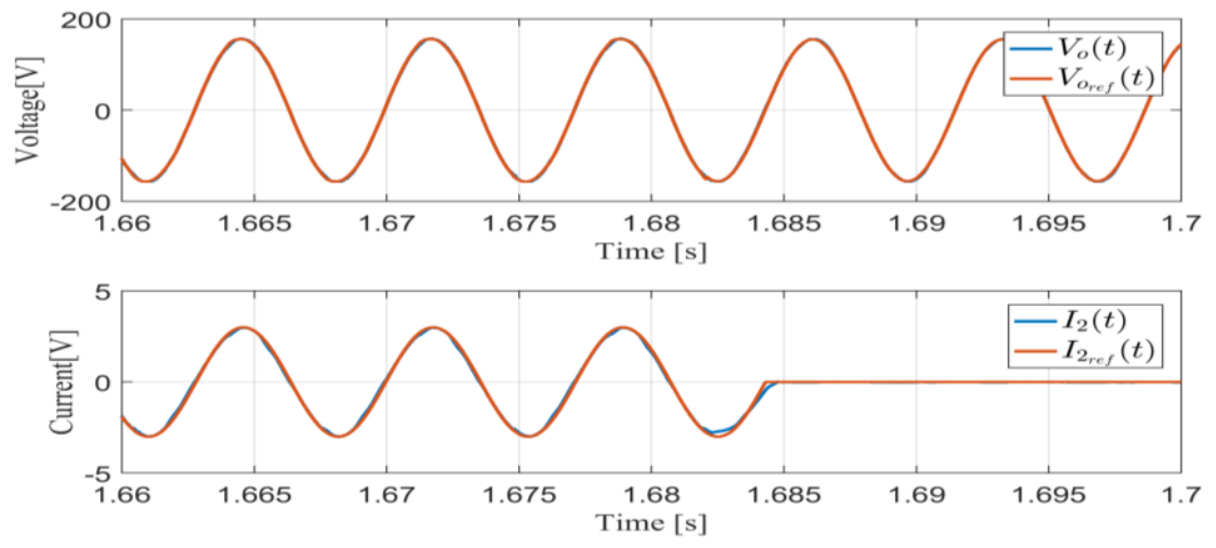

(a)
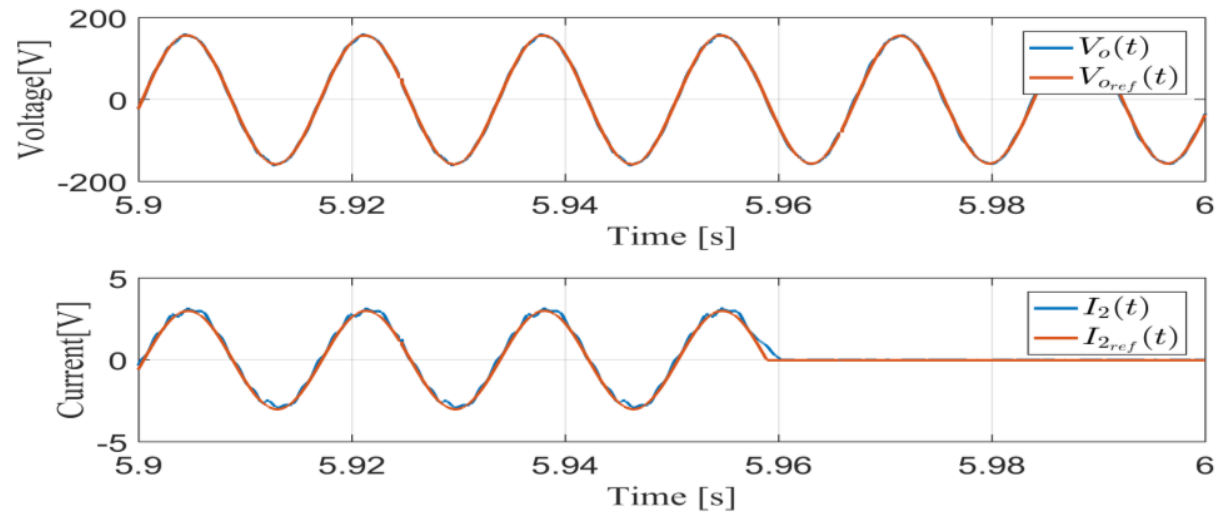

(b)

Figure 0.14 Voltage and Current tracking response during transfer from GC mode to SA mode (a) Proposed Scheme (b) Cascaded PR controller (required gains tuning). 


\subsection{SUMMARY}

A control scheme based on the backstepping control approach has been designed in this chapter to improve the power quality for a single-phase grid-connected inverter system with a local load. The quality of the local load voltage and the grid current is improved simultaneously in the grid-connected mode. The design procedure has been accomplished by using a nonlinear variable structure observer to avoid the numerical derivative of the output voltage and to make the controller robust for different load types. The stability of the proposed controller/observer scheme is demonstrated by using Lyapunov stability analysis. Moreover, the stability analysis shows that all signals in the closed loop system are bounded. An experimental testbed has been implemented to test the steady-state and the transient-state performances of the proposed scheme. The experimental results demonstrate that the proposed controller has excellent tracking performance and it shows robustness of the proposed scheme against changes in operating conditions by comparing this scheme with the standard PR controller. Also, a seamless transition between standalone and grid-connected modes has been achieved without changing the control structure and without any resynchronization scheme. 


\section{CHAPTER 5}

\section{FILTER BASED CONTROL SCHEME DESIGN}

The control scheme that has been designed in chapter 4 requires a variable structure observer to avoid the numerical derivative of the output voltage that generated from the novel second order system dynamics. This observer increases the system complexity and observer loop the control loop which in the observer objective should be met before activating the control loop. Moreover, the approach in the previous chapter is prerequisite the mathematical derivative of the grid voltage. In this chapter, a nonlinear filter-based control approach [41] has been proposed to overcome the aforementioned disadvantages of backstepping control approach mentioned above. The same novel second order from chapter 4 will be used here to design the proposed control scheme to reduce the number of current sensors. Thus, creating a control scheme relies only on the output measurements. The subsequently presented approach will improve the quality of the local load voltage in the grid-connected mode and inject a clean current to the grid simultaneously with the same control structure. Moreover, the proposed approach ensures the seamless transition between stand-alone mode and grid-connected mode without changing the controller structure and without any extra effort to resynchronization the inverter to the grid.

The same experimental test bed that implemented in the chapter 4 has been utilized here to further validate the proposed scheme in real-time. Efficiency have been introduced such that the scheme could be more easily implemented in an embedded platform than the previous control schemes. Moreover, comparisons with a conventional cascaded Proportional-Resonance (PR) control scheme that introduced in the previous chapter is presented in this chapter to show the superiority of the proposed scheme. 
The rest of this chapter is organized as follows. The mathematical model of the singlephase grid-connected inverter with LC filter is presented in Section 5.1 and the second order dynamic model is derived. In Section 5.2 the output voltage and grid current controllers are developed by using the filter-based approach. A Lyapunov stability analysis is performed for the proposed control scheme in Section 5.3. The experimental results in Section 5.4 validate the controller development as well compared performance of this controller to typical control scheme. Concluding remarks are provided in Section 5.5.

\subsection{SYSTEM MODEL}

A single-phase grid-connected inverter with LC filter is shown in Fig.1. The system consists of the following elements: DC power source $V_{d c}, \mathrm{H}$-bridge voltage source inverter (VSI), LC filter $L_{f}, C_{f}$. The ac voltage source $V_{g}$ mimics the grid voltage. To connect the inverter to the grid, $L_{g}$ is considered as a grid interface impedance. A local load has been connected in parallel to the capacitor $C_{f}$. The mathematical differential equations representing the system dynamics are obtained by applying Kirchhoff's voltage and current laws to the average model of the inverter system

$$
\begin{gathered}
L_{f} \dot{I}_{1}=V_{d c}\left(D+d_{0}\right)-V_{o} \\
C_{f} \dot{V}_{o}=I_{1}-I_{2}-I_{o} \\
L_{g} \dot{I}_{2}=V_{o}-V_{g}
\end{gathered}
$$

where $I_{1}(t), V_{o}(t), I_{2}(t) \in \mathbb{R}$ are the filter inductor current, capacitor (output) voltage, and injected current to the grid, respectively. $I_{o} \in \mathbb{R}$ is the current of the local load. $D(t) \in$ $(-1,1)$ is duty ratio of the switching signal. $d_{0} \in \mathbb{R}$ is an unknown disturbance representing the dead-time and voltage drop effects in the switching devices. 
The second order dynamic equation of the system is obtained after substituting (1) and (3) in the time derivative of (2) as

$$
\ddot{V}_{o}=a V_{o}+b V_{g}+c D+c d_{0}-\frac{1}{C_{f}} \dot{I}_{o}
$$

where $a=-\left(\frac{1}{L_{f} C_{f}}+\frac{1}{L_{g} C_{f}}\right), b=\frac{1}{L_{g} C_{f}}, c=\frac{V_{d c}}{L_{f} C_{f}}$.

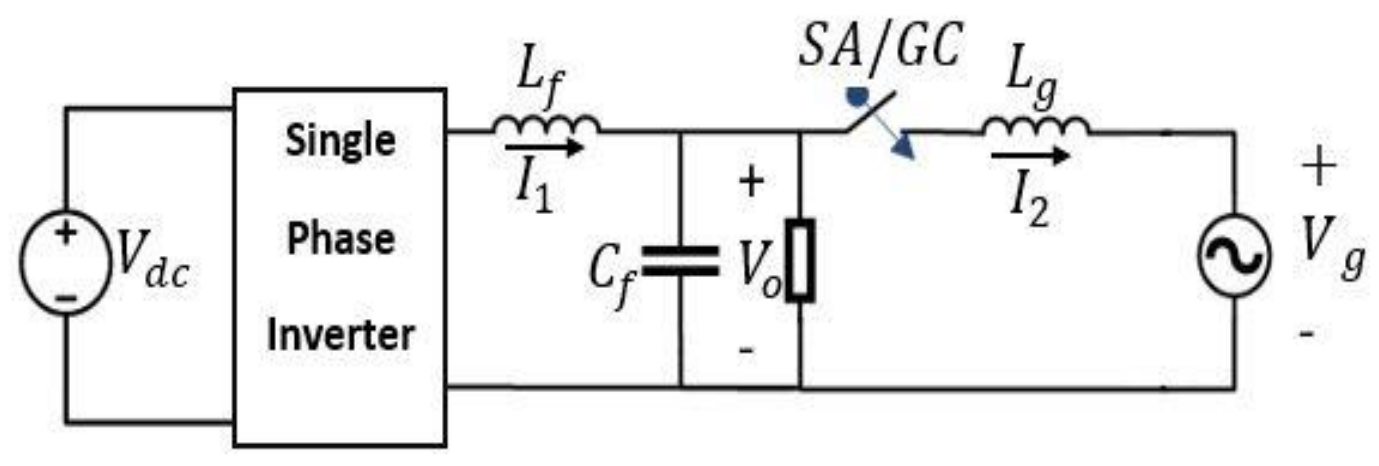

Figure 0.1 Single-phase grid-connected inverter system

\subsection{CONTROL DEVELOPMENT}

The subsequent development of this control is based upon the following assumptions:

Assumption 1: $L_{g}, L_{f}, C_{f}, V_{d c}$ are known a priori, constant system parameters.

Assumption 2: The output voltage $V_{o}(t)$, and the grid current $I_{2}(t)$ are measurable.

Assumption 3: The load current $I_{o}(t)$ and its derivative $\dot{I}_{o}(t)$ are bounded, hence $\left|I_{o}\right|,\left|\dot{I}_{o}\right| \in \mathcal{L}_{\infty}$

Assumption 4: The unknown disturbance $d_{0}$ is bounded and slowly time-varying, hence $\dot{d}_{0}(t) \approx 0$

Assumption 5: The grid voltage $V_{g}(t)$ is measurable and bounded up to its second time derivative. 
The voltage control objective of the proposed controller is to maintain low THD of the local load voltage $V_{o}(t)$ regardless of the type of load and achieve the reference output voltage trajectory $V_{o_{\text {ref }}}(t)$, hence $V_{o}(t) \rightarrow V_{o_{\text {ref }}}(t)$ as $t \rightarrow \infty$. Also, the current control objective is exchanging clean current with the grid by regulating the grid current $I_{2}(t)$ to a predefined reference current $I_{2_{\text {ref }}}(t)$, hence $I_{2}(t) \rightarrow I_{2_{\text {ref }}}(t)$ as $t \rightarrow \infty$. To guarantee unity power factor at the grid side, the reference grid current should be completely sinusoidal and in phase with the grid voltage

$$
I_{2_{\text {ref }}} \triangleq I_{2 p} \sin \theta
$$

where $I_{2 p}$ is the peak value of the reference grid current which is selected by the user. Also, $\theta$ is the phase angle of the grid voltage. This angle could be estimated based on any PhaseLocked Loop (PLL) scheme form the literature. In this work, a second order generalized integrator is used to create an orthogonal signal generator-based phase-locked loop (SOGIOSG PLL) has been used due to its robustness and good tracking performance as we have seen in chapter 4.

The filter-based approach uses a set of error dynamics which are motivated by the stability analysis in order to meet the output feedback control objectives. The following tracking error signals, $e_{2}(t), e(t)$ and filtered error signals, $r_{f}(t), e_{f}(t)$ are defined

$$
\begin{gathered}
e_{2} \triangleq I_{2_{r e f}}-I_{2} \\
e \triangleq V_{o_{r e f}}-V_{o} \\
r_{f} \triangleq p+\left(K_{2}+\alpha\right) e \\
\dot{e}_{f} \triangleq-\alpha e_{f}+r_{f}
\end{gathered}
$$


where $K_{1}, K_{2}, \alpha$ are positive gains, and $p(t)$ is an auxiliary variable defined for filter implementation as follows

$$
\dot{p} \triangleq-K_{1} r_{f}+\left(K_{2}+\alpha\right)\left(\alpha e-r_{f}\right)-e-e_{f} .
$$

To start the control development, the error dynamic between the actual error signal $\dot{e}(t)+$ $\alpha e(t)$ and the filtered one $r_{f}(t)$ from (9) is defined as

$$
\eta \triangleq \dot{e}+\alpha e-r_{f}
$$

To design the $V_{o_{\text {ref }}}(t)$ that will regulate the injected current to the grid, we start by taking the time derivative of (6), pre-multiplying by $L_{g}$, substituting the system equation from (3), and using (7) to obtain the following open-loop error dynamics for $e_{2}(t)$

$$
L_{g} \dot{e}_{2}=L_{g} \dot{I}_{2_{r e f}}+e-V_{o_{r e f}}+V_{g}
$$

From (12) we can design the auxiliary control signal $V_{o_{r e f}}$ to regulate $I_{2}$ as

$$
V_{o_{\text {ref }}} \triangleq L_{g} \dot{I}_{2_{\text {ref }}}+V_{g}+K_{g} e_{2}
$$

where $K_{g} \in \mathbb{R}^{+}$is a control gain. After substituting (13) into (12), the closed loop error system for $e_{2}(t)$ is obtained

$$
L_{g} \dot{e}_{2}=e-K_{g} e_{2}
$$

Taking the second time derivative of (13), using $\dot{e}_{2}(t)$ from (14), recalling $\dot{e}(t)$ from (11), $\ddot{V}_{o_{\text {ref }}}$ can be obtained after some simplifications to use it later as

$$
\ddot{V}_{o_{r e f}}=L_{g} \dddot{I}_{2_{r e f}}+\ddot{V}_{g}+\frac{K_{g}}{L_{g}}\left(\eta+r_{f}\right)+A e+\frac{K_{g}^{3}}{L_{g}^{2}} e_{2}
$$

where $A=-\frac{K_{g}}{L_{g}}\left(\frac{K_{g}}{L_{g}}+\alpha\right)$.

To meet the voltage control objective, we start with the time derivative of (11) 


$$
\dot{\eta}=\ddot{e}+\alpha \dot{e}-\dot{r}_{f}
$$

To proceed we need the time derivative of $r_{f}(t)$ from (8) as follows

$$
\dot{r}_{f}=\dot{p}+\left(K_{2}+\alpha\right) \dot{e} .
$$

By substituting $\dot{p}(t)$ from (10), $\dot{e}(t)$ from (11) into (17) and after simplification, the following expression is obtained

$$
\dot{r}_{f}=-K_{1} r_{f}+\left(K_{2}+\alpha\right) \eta-e-e_{f}
$$

By using (18) and the second time derivative of (7) into (16), the following equation is obtained

$$
\dot{\eta}=\ddot{V}_{o_{r e f}}-\ddot{V}_{o}-\alpha^{2} e+\alpha r_{f}+K_{1} r_{f}-K_{2} \eta+e+e_{f}
$$

Substituting (15) and the system dynamic from (4) into (19) and after some mathematical simplifications, the open loop error dynamics for $\eta(t)$ can be obtained as follows

$$
\begin{gathered}
\dot{\eta}=L_{g} \dddot{I}_{2_{r e f}}+\frac{K_{g}}{L_{g}}\left(\eta+r_{f}\right)+A e+\frac{K_{g}^{3}}{L_{g}^{2}} e_{2}-a V_{o} \\
-b V_{g}-c D-\alpha^{2} e+\alpha r_{f}+K_{1} r_{f}-K_{2} \eta+e+e_{f}+N_{0}
\end{gathered}
$$

where $N_{0}=\ddot{V}_{g}-c d_{0}+\frac{1}{C_{f}} \dot{I}_{o}$ is a lumped time-varying uncertainty. Based on Assumptions 3-4 it can be concluded that $N_{0}$ is bounded. From the open loop error dynamic of $\eta(t)$ in (20) and motivated by the subsequent stability analysis, the duty ratio control signal is designed as

$$
\begin{gathered}
D \triangleq \frac{1}{c}\left[L_{g} \dddot{I}_{2_{r e f}}-a V_{o}-b V_{g}+\frac{K_{g}}{L_{g}} r_{f}+A e+\frac{K_{g}^{3}}{L_{g}^{2}} e_{2}\right. \\
\left.-\alpha^{2} e+\alpha r_{f}+K_{1} r_{f}+e+e_{f}+K_{3} \operatorname{sgn}\left(e-e_{f}\right)+e+\left(K_{2}+\alpha\right) r_{f}\right]
\end{gathered}
$$

where $K_{3} \in \mathbb{R}^{+}$is a positive constant gain selected based on the stability analysis, $\operatorname{sgn}(\cdot)$ is the standard signum function which is used to compensate for uncertainty $N_{0}$. Finally, 
by substituting the control signal from (21) into (20), the closed loop error dynamic for $\eta(t)$ can be obtained as

$$
\dot{\eta}=-\left(K_{2}-\frac{K_{g}}{L_{g}}\right) \eta-K_{3} \operatorname{sgn}\left(e-e_{f}\right)-e-\left(K_{2}+\alpha\right) r_{f}+N_{0}
$$

\subsection{STABILITY ANALYSIS}

Before introducing the main theorem, the following two lemmas should be stated to be utilized later.

Lemma 1. Define the auxiliary function $L_{0}(t) \in \mathbb{R}$ as follows

$$
L_{0} \triangleq \eta\left(N_{0}-K_{3} \operatorname{sgn}\left(e-e_{f}\right)\right)
$$

If the control gain $K_{3}$ is chosen to meet the following condition

$$
K_{3}>\left|N_{0}\right|+\left|\dot{N}_{0}\right|
$$

then,

$$
\beta_{0} \geq \int_{t_{0}}^{t} L_{0}(\sigma) d \sigma
$$

where $\sigma$ is a dummy variable of integration and $\beta_{0}$ is a positive constant defined as

$$
\beta_{0}=K_{3}|e(0)|-e(0) N_{0}(0)
$$

Proof: The proof of Lemma 1 is provided in Appendix 1 in [52].

Lemma 2. Let the region $\mathfrak{D}$ to be defined as $\mathfrak{D} \triangleq\left[y \in \mathbb{R}^{m}\|y\|<\epsilon\right]$, where $\epsilon$ is positive constant, and let $V(t, y): \mathbb{R}^{+} \times \mathfrak{D} \rightarrow \mathbb{R}^{+}$be a continuously differentiable function such that

$$
W_{1}(y) \leq V(y) \leq W_{2}(y), \dot{V}(y) \leq-W(y)
$$

$\forall t \geq 0$ and $\forall y \in \mathfrak{D}$ where $W_{1}(y), W_{2}(y)$ are continuous positive functions and $W(y)$ is a uniformly continuous positive semi-define function. If the condition in (27) is met and $y(0) \in \mathfrak{J}$, the result is 


$$
W(y(t)) \rightarrow 0 \text { as } t \rightarrow \infty
$$

where the region $\mathfrak{I}$ is defined as follows

$$
\mathfrak{I} \triangleq\left[y \in \mathfrak{D} \mid W_{2}(y) \leq \delta\right]
$$

where $\delta<\min W_{1}(y)$ is a positive constant.

Proof: See Theorem 8.4 in [20] for Lemma 2 proof.

Theorem 1: The control law in (21) ensures that all closed loop error signals are bounded and regulated as $e_{2}(t), e(t), e_{f}(t), r_{f}(t), \eta(t) \rightarrow 0$ as $t \rightarrow \infty$, provided $K_{3}$ is selected to satisfy (24).

Proof: Let define the auxiliary function $P_{0}(t) \in \mathbb{R}$ as

$$
P_{0} \triangleq \beta_{0}-\int_{t_{0}}^{t} L_{0}(\sigma) d \sigma
$$

From Lemma 1 it is clear that $P_{o}(t) \geq 0$. Now, Lyapunov function $V(t, y) \in \mathbb{R}$ can be defined as

$$
V \triangleq \frac{L_{g}}{2} e_{2}^{2}+\frac{1}{2} e^{2}+\frac{1}{2} e_{f}^{2}+\frac{1}{2} r_{f}^{2}+\frac{1}{2} \eta^{2}+P_{0}
$$

where $y=\left[\begin{array}{ll}z^{T} & \sqrt{P_{0}}\end{array}\right]^{T}$ and $z=\left[\begin{array}{llll}e_{2} & e & e_{f} & r_{f}\end{array}\right]$. The function in (31) can be lower and upper bounded as

$$
\lambda_{1}\|y\|^{2} \leq V(y) \leq \lambda_{2}\|y\|^{2}
$$

where $\lambda_{2}>\lambda_{1}$ and both are positive constants. The time derivative of (31) is

$$
\dot{V}=L_{g} e_{2} \dot{e}_{2}+e \dot{e}+e_{f} \dot{e}_{f}+r_{f} \dot{r}_{f}+\eta \dot{\eta}-L_{0}
$$

After substituting the error dynamics from (9), (11), (14), (18) and (22) into (33), the following expression can be obtained where (23) is also utilized 


$$
\dot{V}=-K_{g} e_{2}^{2}-\alpha e^{2}-\alpha e_{f}^{2}-K_{1} r_{f}^{2}-\left(K_{2}-\frac{K_{g}}{L_{g}}\right) \eta^{2}+e_{2} e
$$

The previous expression can be upper bounded and simplified by using the triangle inequality $\left|e_{2}\right||e| \leq \frac{1}{2}\left|e_{2}\right|^{2}+\frac{1}{2}|e|^{2}$ as

$$
\dot{V} \leq-\left(K_{g}-\frac{1}{2}\right)\left|e_{2}\right|^{2}-\left(\alpha-\frac{1}{2}\right)|e|^{2}-\alpha e_{f}^{2}-K_{1} r_{f}^{2}-\left(K_{2}-\frac{K_{g}}{L_{g}}\right) \eta^{2}
$$

If the following gain conditions in (36) are met, it is easy to rewrite (35) as in (37)

$$
\begin{gathered}
K_{g}>\frac{1}{2}, \quad \alpha>\frac{1}{2}, \quad K_{2}>\frac{K_{g}}{L_{g}} . \\
\dot{V} \leq-\gamma\|z\|^{2}
\end{gathered}
$$

where $\gamma$ is a positive constant. By applying Lemma 2 to (32) and (37) it is concluded that the lower and upper bounds of $V(t)$ are given as follows

$$
W_{1}(y)=\lambda_{1}\|y\|^{2}, W_{2}(y)=\lambda_{2}\|y\|^{2}
$$

and the upper bound for $\dot{V}(t)$ is defined by using (37) as follows

$$
W(y)=\gamma\|z\|^{2}
$$

Again Lemma 2 can be utilized along with (36) and (37) to define the region $\mathfrak{D}$ as follows

$$
\mathfrak{D} \triangleq\left[y \in \mathbb{R}^{5}\|y\|<\max \left(K_{g}, \alpha, K_{2}\right)\right]
$$

From (31) and (36) it is clear that $V(t, y(t))$ is bounded, $V(t, y(t)) \in \mathcal{L}_{\infty}$; hence, $e_{2}(t), e(t), e_{f}(t), r_{f}(t), \eta(t) \in \mathcal{L}_{\infty}$. From (11) it is clear that $\dot{e}(t) \in \mathcal{L}_{\infty}$. From (9), (10), and (18) it is concluded $\dot{e}_{f}(t), \dot{p}(t), \dot{r}_{f}(t) \in \mathcal{L}_{\infty}$. Since $e_{2}, V_{g}, \dot{I}_{2_{r e f}} \in \mathcal{L}_{\infty}$, we can see from (13) that $V_{\text {ref }}(t) \in \mathcal{L}_{\infty}$. From (7) and $V_{\text {ref }}(t) \in \mathcal{L}_{\infty}$, therefore $V_{o}(t) \in \mathcal{L}_{\infty}$. Since all the signals in (21) are bounded, we can say that $D(t) \in \mathcal{L}_{\infty}$. From (4), (15), Assumptions(2-4) and based on the above results we can see that $\ddot{V}_{o}(t), \ddot{V}_{\text {oref }}(t) \in \mathcal{L}_{\infty}$. Using the above 
results and based on (38) one can conclude that $\dot{W}(y(t)) \in \mathcal{L}_{\infty}$, which is a sufficient condition to say that $W(y(t))$ is uniformly continuous. If the region $\mathfrak{\Im}$ is defined as follows

$$
\mathfrak{J} \triangleq\left[y \in \mathfrak{D} \mid W_{2}(y)<\lambda_{1}\right]
$$

Lemma 2 can be invoked to say that $\gamma\|z(t)\|^{2} \rightarrow 0$ as $t \rightarrow \infty \forall y(0) \in \mathfrak{I}$. From the above and the definition of $z(t)$ it is clear $e_{2}(t), e(t), e_{f}(t), r_{f}(t), \eta(t) \rightarrow 0$ as $t \rightarrow \infty \forall y(0) \in$ I.

\subsection{EXPERIMENTAL RESULTS}

Real-time implementation has been developed as shown in Fig.2 to evaluate the performance of the proposed controller-observer scheme. The main purpose of this work is to control the inverter grid side, For this reason, a programmable DC power supply BKPRECISION XLN30052 1.56KW can be used as input DC voltage of the H-bridge singlephase inverter [53]. A two-quadrant programmable AC source BK- PRECISION 9803 750VA was used to emulate the utility grid at fundamental frequency $60 \mathrm{~Hz}$. A power resistor $\left(R_{p}\right)$ has been placed in parallel with this $\mathrm{AC}$ source and sized such that its power draw exceeds the power which is sourced by the inverter (as set by the value of $I_{2 p}$ ). This is done to ensure that current is never sent to the AC source. The NI CompactRIO 9063 with LabVIEW software has been used to implement the proposed algorithm and to execute it in real-time by the onboard Virtex-5 LX50 FPGA. The control diagram of the proposed scheme is shown in Fig.3. Table I summarizes the system parameters and the controller gains. The minimum value for each control gain has been selected based on the conditions in (36). An iterative process with some experimental trails is required to choose the gains that give the best performance. While there is not a closed-form solution for gain determination other than the gain conditions from (36), the gain selection is not sensitive 
for a reasonable result. As a result, the gains in Table 1 have been selected for best performance. Both the steady state and transient performances of the proposed scheme have been tested while the inverter is injecting current to the grid and supporting different types of the local loads in the same time. For comparison purposes, the well-known Proportional-Resonance (PR) controller has been utilized from previous chapter.

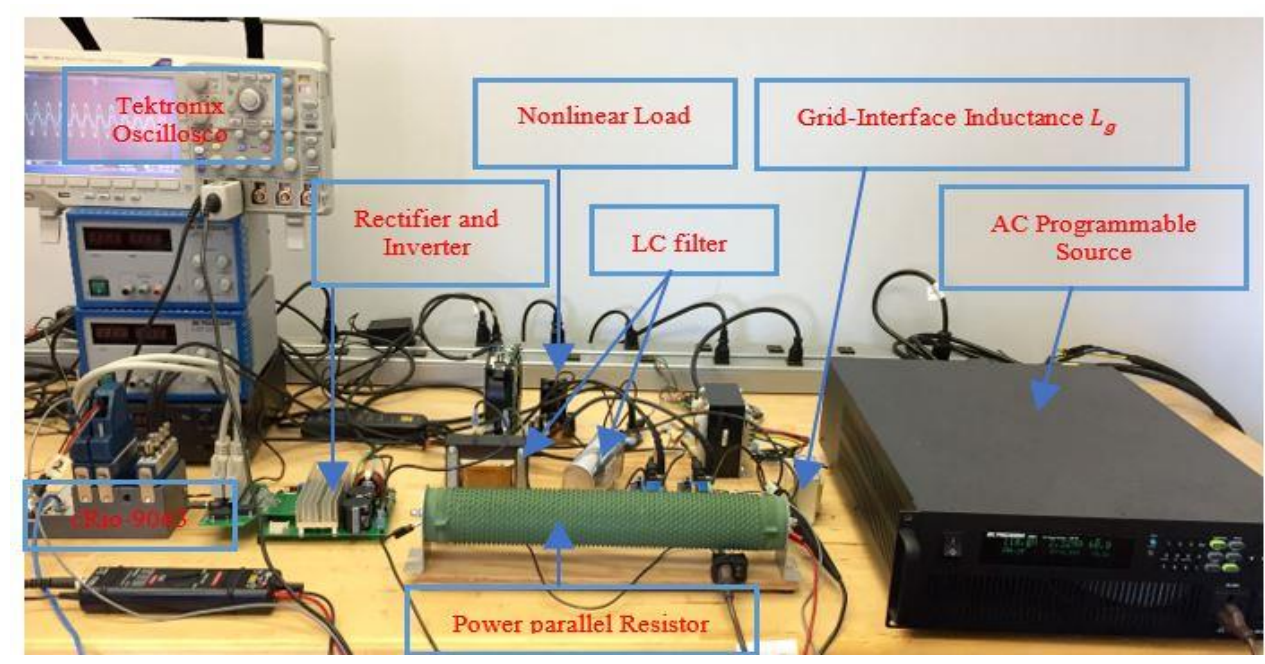

Figure 0.2 Experimental setup of the grid-connected inverter

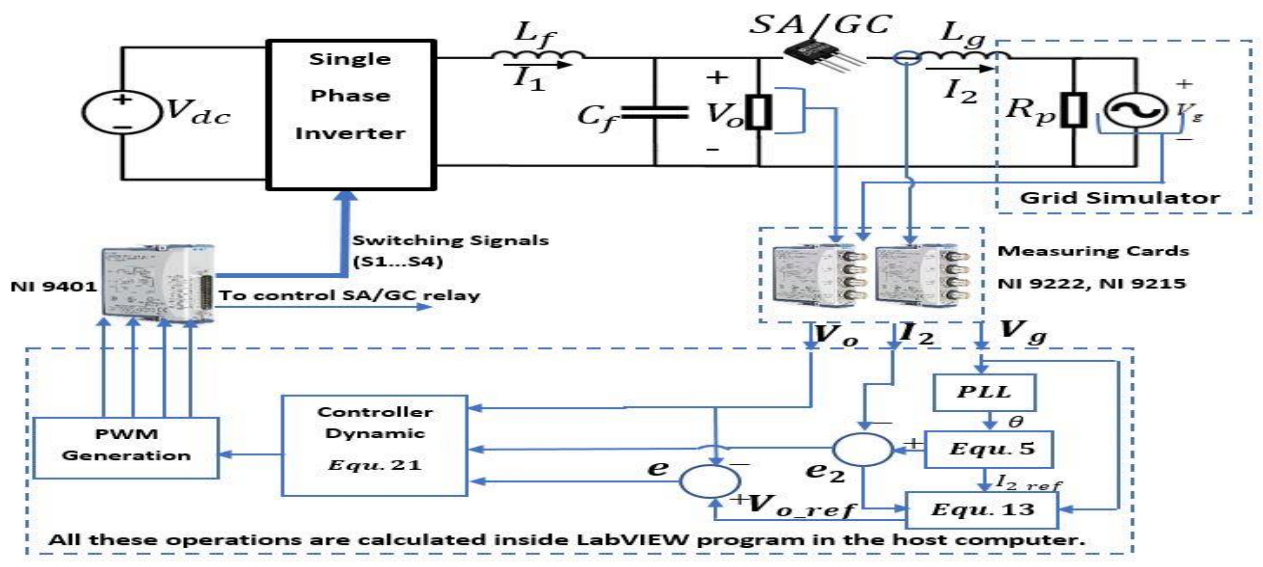

Figure 0.3 Block diagram for the proposed control scheme

In the first experiment the inverter is connected to the grid while the resistive-inductive (RL) local load is supplied power from the inverter. The amount of the injected power to the grid is determined by choosing the value of the reference grid current. In this test, the 
peak value of the reference current in (5) is selected to be 3A. Fig. 4 shows the steady-state tracking performance of the local load voltage $V_{o}(t)$ for both the proposed scheme and the cascaded PR controller. The injected grid current $I_{2}(t)$ response for both schemes are shown in Fig.5. The gains for the cascaded PR control scheme in this test are shown in Table 1. The percentage of the rms steady-state errors and the THD for both controllers Table 0.1 System and control parameters

\begin{tabular}{|c|c|c|c|c|c|c|}
\hline$L_{f}[m H]$ & $C_{f}[\mu F]$ & $L_{g}[\mathrm{mH}]$ & $V_{d c}[V]$ & $f_{s w}\left[K_{z}\right]$ & $f_{s}\left[K_{z}\right]$ & $V_{g}\left[V_{r m s}\right]$ \\
\hline 10 & 50 & 2.5 & 300 & 16 & 32 & 110 \\
\hline$K_{1}$ & $K_{2}$ & $K_{3}$ & $\alpha$ & $K_{g}$ & & \\
\hline 3,000 & 13,000 & 13,000 & 20,000 & 16 & & \\
\hline Local & \multirow{2}{*}{ RL } & $R[\Omega]$ & $L[\mathrm{mH}]$ & \multirow{2}{*}{ NL } & $R[\Omega]$ & $C[\mu F]$ \\
\hline loads & & 150 & 32 & & 250 & 220 \\
\hline \multicolumn{7}{|c|}{ Cascaded PR Control Scheme gains for RL load } \\
\hline \multirow{2}{*}{ Inner } & $k_{i i}$ & $k_{p i}$ & \multirow{2}{*}{ Outer } & $k_{i o}$ & $k_{p o}$ & \\
\hline & 50 & 7 & & 10 & 1 & \\
\hline
\end{tabular}

are summarized in Table 2. Total harmonic distortion in the voltages and currents are used to assess the power quality for the inverter systems that are connected to the utility grid. The maximum THD for the voltage and current for such systems is 5\% [4]. Tektronix DPO 3014 digital oscilloscope was used in this work to measure the THD for both the voltage and current. The control input $D(t)$ in this case is shown in Fig.6. Since the proposed novel control scheme has been compared to the PR scheme, the performance assessment needs to be performed for both control objectives. With regard to the voltage control objective, the cascaded PR-scheme has a good tracking performance in the steady-state operation for RL local load voltage in term of the steady-state error, as well as the THD of PR-scheme is low in this case as shown in Table 2. The above results show that the PR-scheme has 
been tuned very well for this specific operating point. For the current control objective, the proposed control scheme performance is better than the PR-scheme in the terms of the steady-state errors and THD.

In the second experiment, the performance of the proposed scheme and the cascaded PR control scheme has been tested when the nonlinear (NL) local load is connected to the inverter while the inverter is connected to the grid. The cascaded PR control scheme failed to deal with the changing of the local load type which leads the system to be unstable. To complete the comparison, a gain tuning process has been completed to return the system to the stability region and to minimize the steady-state error. The new gains are obtained to be $k_{i i}=200, k_{p i}=17, k_{i o}=100, k_{p o}=1$. Fig. 7 and 8 show the tracking performance of the proposed and the cascaded PR controllers for the local load voltage and the injected grid current, respectively. Table 2 shows that the proposed scheme outperforms cascaded PR scheme in the percentage rms steady-state error as well as the THD for both the local load voltage and the injected current to the grid while the inverter supplies power to the nonlinear local load and to the grid simultaneously. Fig. 9 demonstrates unity power factor at the grid side even with the highly distorted local load current. From the previous results, it is clear that the proposed scheme is injecting very clean current to the grid while supporting the nonlinear local load with low THD voltage. Also, the proposed scheme is robust for the changing local load type, but the cascaded PR controller needs tuning the gains for each local load type. In summary, the PR-scheme needs different sets of gains, one set for each type of load. In comparison, the proposed control scheme is robust for the local load type changes. 
To test the transient performance for the proposed scheme and the cascaded PR scheme, a step change in the amplitude of the reference grid current from $2 \mathrm{~A}$ to $3 \mathrm{~A}$ is applied during grid-connected mode while the inverter sends power to the nonlinear local load as well. This step is applied at the peak point in order to illustrate the worst operation. Fig. 10 shows that the grid current almost immediately follows the reference grid current for the proposed scheme. For the cascaded PR controller, the current needs around 16 cycles to follow its reference.

In experiment 4 , transition event from the stand-alone to grid-connected modes and vice versa is occurred for the inverter. The SA/GC switch in Fig.3 (CPC1998J solid state relay) is controlled by LABVIEW command to do this transition. The voltage and current responses during the transition from the SA mode into the GC mode are shown in Fig.11 while the inverter supplies current to the nonlinear local load and the reference grid current $I_{2 p}$ is set to be $3 \mathrm{~A}$. By opening the $\mathrm{SA} / \mathrm{GC}$ switch and changing the reference current to zero the inverter will work in the SA mode as shown in Fig.12. It is shown from Fig 11 and 12 a seamless transition in the output voltage is ensured by the proposed scheme. Also, the proposed scheme has very fast and smooth response in the transition between the two operation modes. On the other hand, the current needs about 20 cycles to reach the steadystate current for the cascaded PR controller. Based on the above results, we can see that with one control structure, the proposed controller is working efficiently in SA and GC operation modes.

To validate the proposed control scheme under distorted grid, a hardware-in-the-loop setup has been prepared as shown in Fig. 13. The Typhoon HIL 603 hardware has been used to emulate the single-phase grid-connected inverter system with nonlinear local load. The 
proposed control scheme is implemented in the LABVIEW FPGA as was completed for the experimental results. Fig. 14 demonstrates the distorted grid with the THD is $17 \%$ and the spectrum analysis that shows the grid has third, fifth, and seventh harmonics with value for each harmonic is 15\%,10\%, and 5\% respectively. Fig. 15 shows the injected current to the grid with $2.3 \%$ THD and the percentage of the root mean squared (RMS) error for the injected current to the grid is $2.79 \%$. From these results it is clear that the proposed control scheme is also effective under the distorted grid conditions.
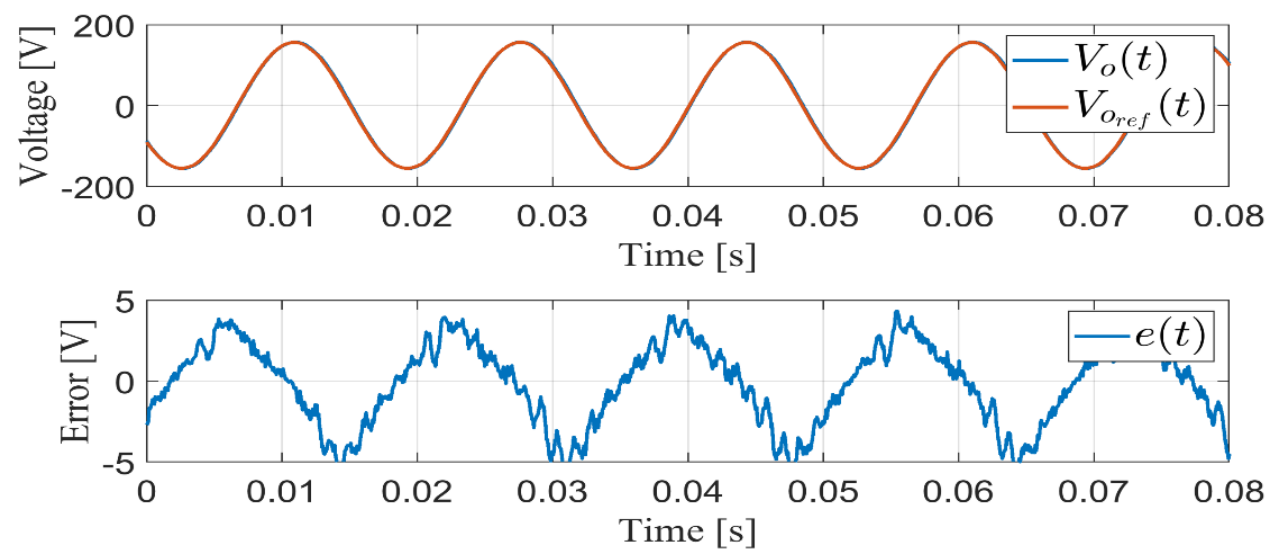

(a)
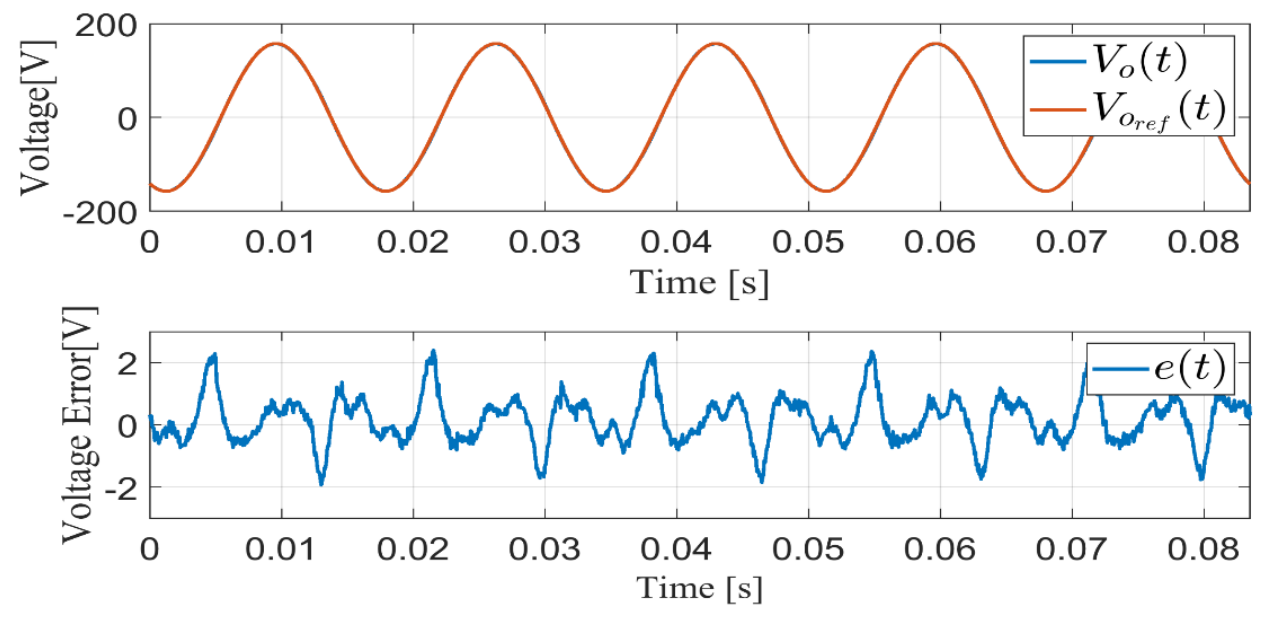

(b)

Figure 0.4 Tracking response of the output voltage for RL local load (a) Proposed Scheme, (b) Cascaded PR scheme 

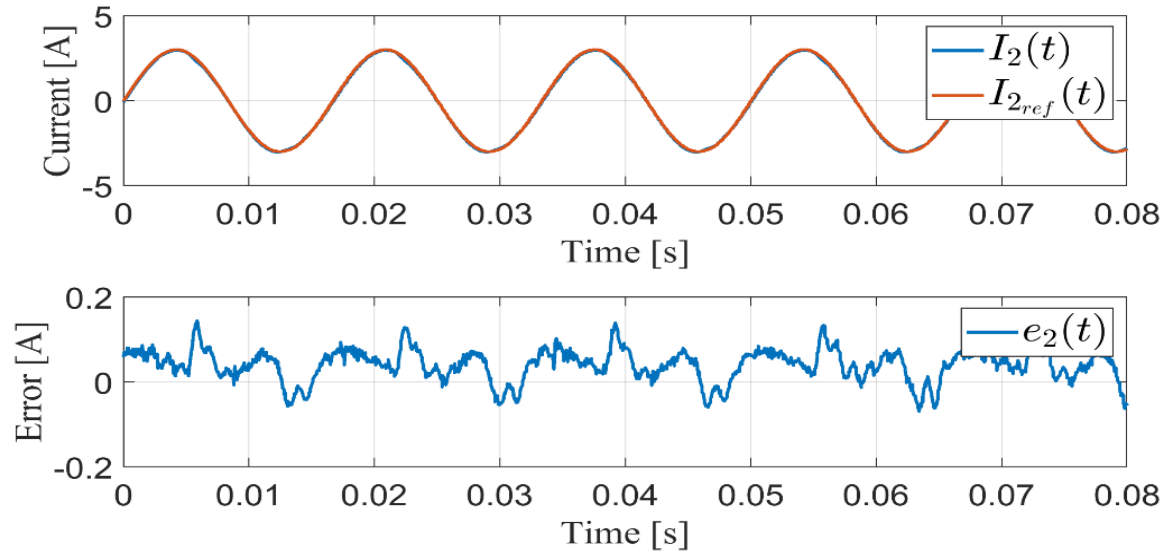

(a)
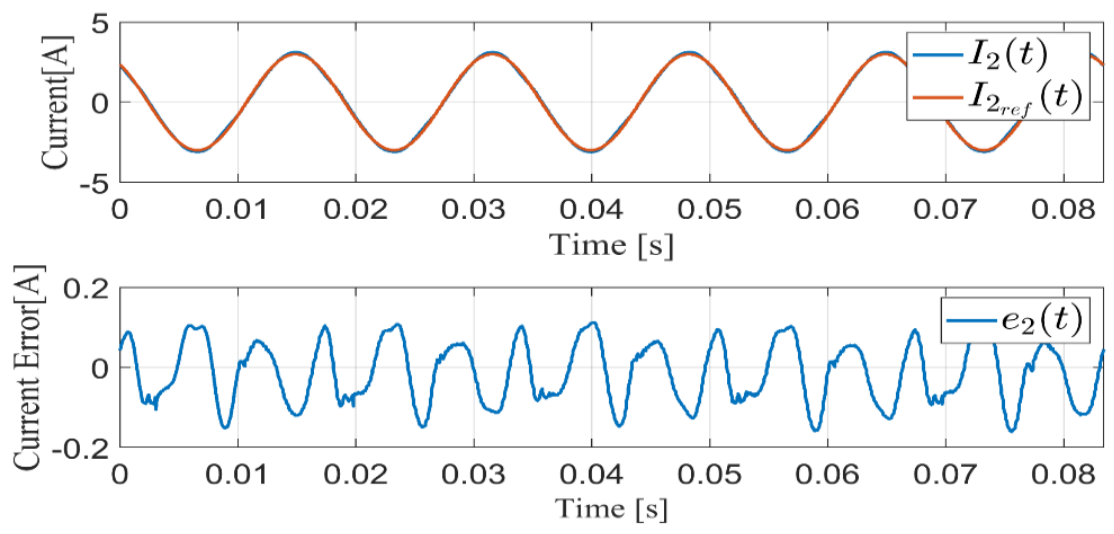

(b)

Figure 0.5 Tracking response of the grid current under RL local load (a) Proposed Scheme, (b) Cascaded PR scheme

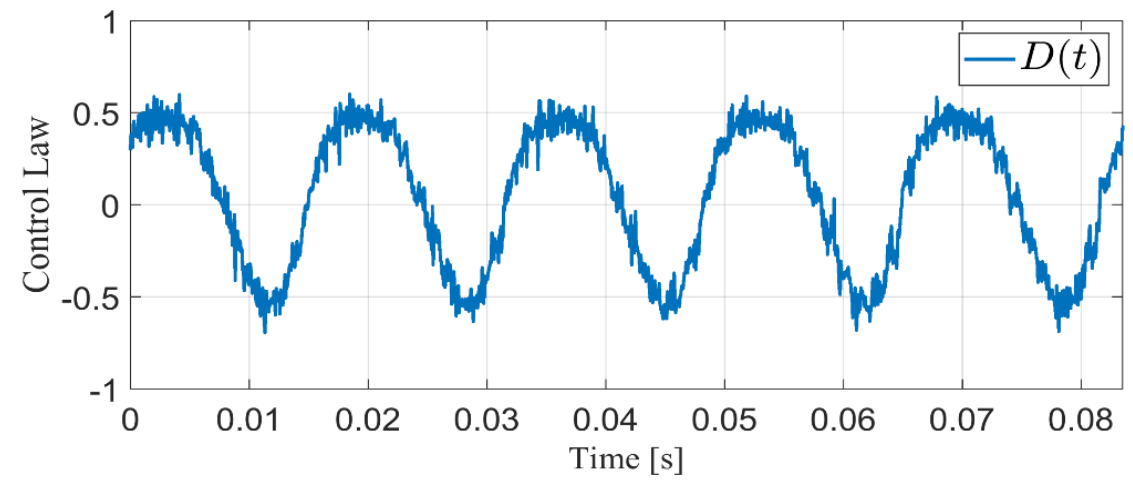

Figure 0.6 Control law for the proposed scheme under RL local load 

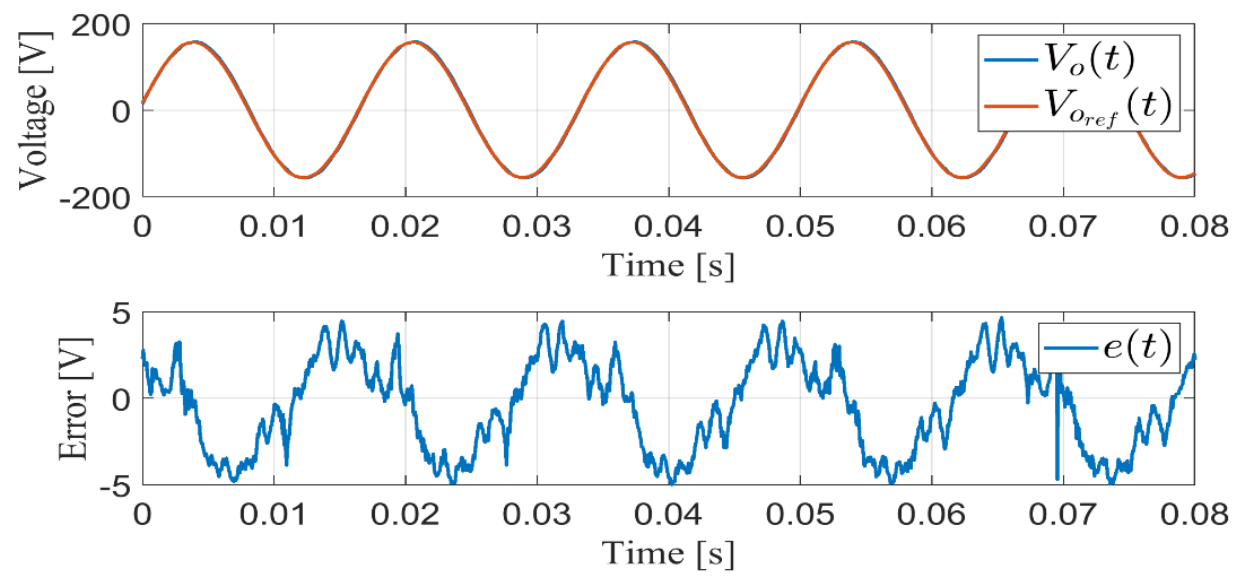

(a)

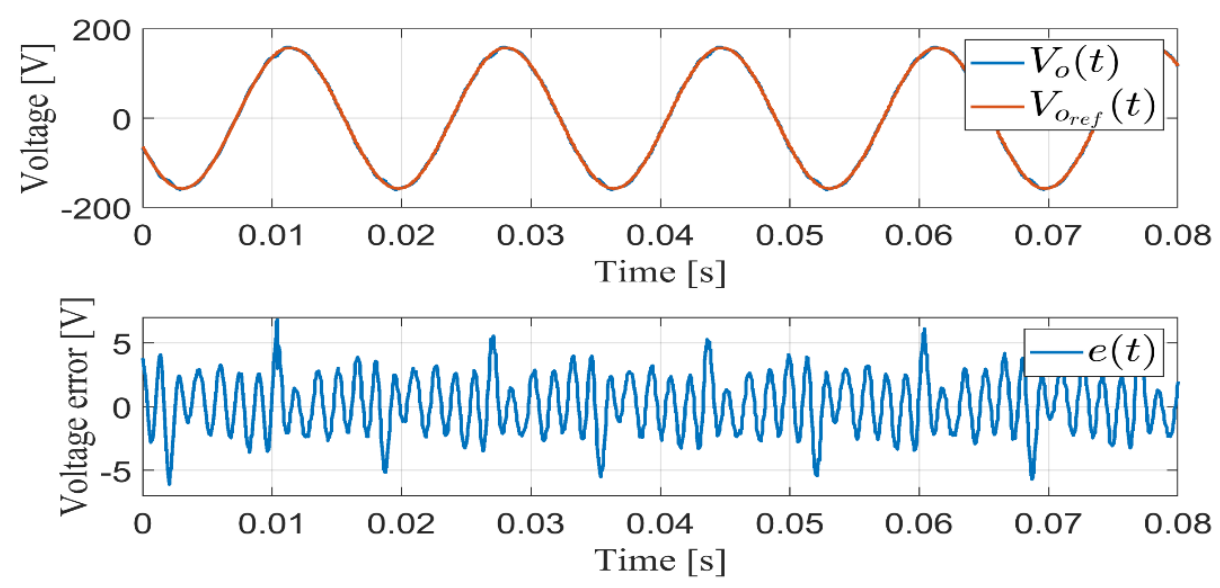

(b)

Figure 0.7 Tracking response of the output voltage for NL local load. (a) Proposed Scheme, (b) Cascaded PR scheme (required gains tuning).

$\underline{\text { Table 0.2 RMS Error and THD }}$

\begin{tabular}{|c|c|c|c|c|c|c|c|}
\hline \multicolumn{4}{|c||}{ RL Load } & \multicolumn{4}{c|}{ NL Load } \\
\hline $\boldsymbol{e}_{\boldsymbol{r m s} \boldsymbol{s}} \%$ & \multicolumn{1}{|c|}{ THD\% } & \multicolumn{2}{|c|}{$\boldsymbol{e}_{\boldsymbol{r m s}} \%$} & \multicolumn{2}{c|}{ THD\% } \\
\hline V & I & V & I & V & I & V & I \\
\hline \multicolumn{7}{|c|}{ Proposed Scheme } \\
\hline 1.64 & 1.82 & 0.77 & 1.96 & 1.69 & 2.49 & 0.75 & 2.5 \\
\hline \multicolumn{7}{|c|}{ PR Scheme (required gains tuning) } \\
\hline 0.7 & 3.6 & 0.56 & 4 & 1.98 & 6.66 & 2.12 & 5.84 \\
\hline
\end{tabular}



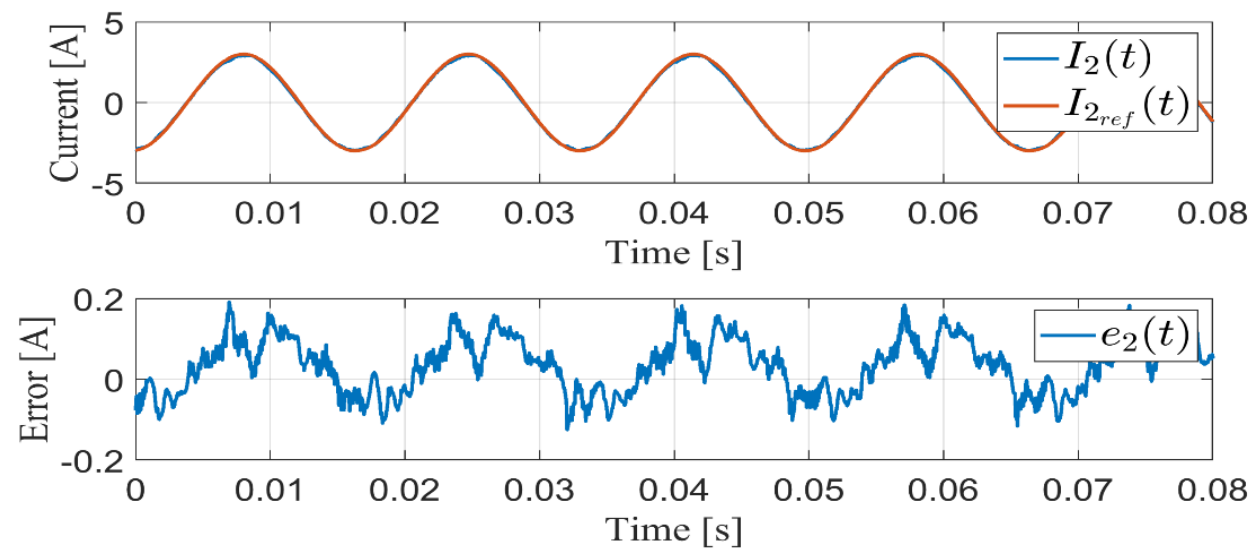

(a)
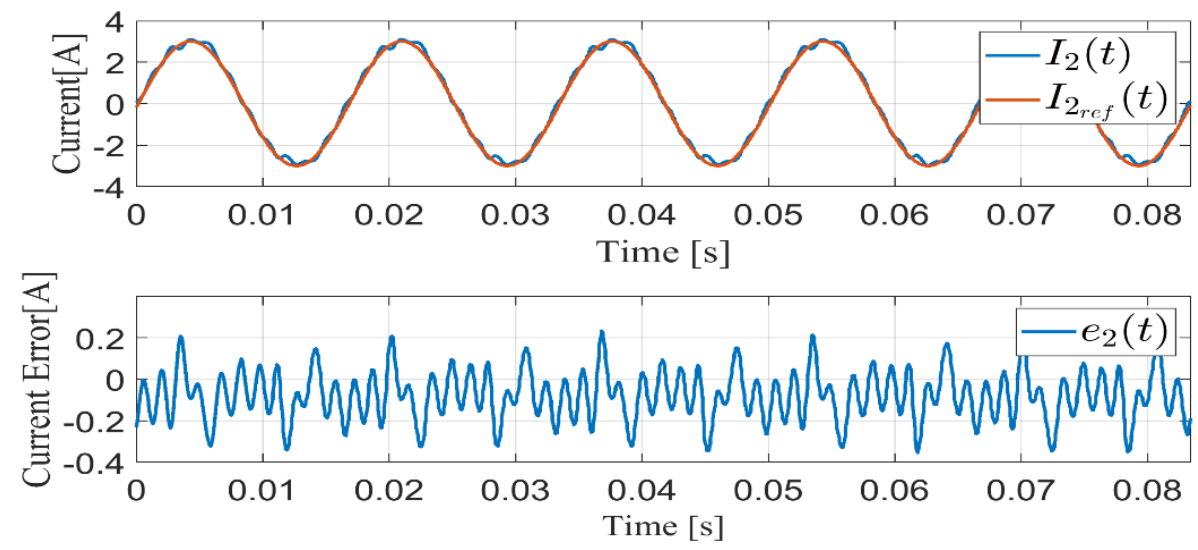

(b)

Figure 0.8 Tracking response of the grid current under NL local load. (a) Proposed Scheme, (b) Cascaded PR scheme (required gains tuning)

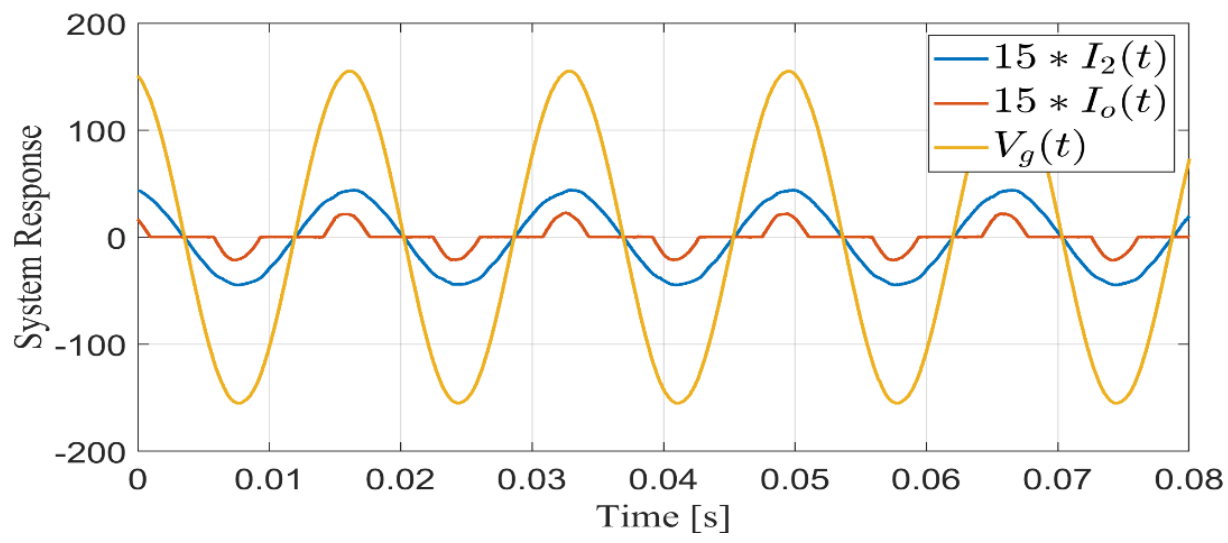

Figure 0.9 System response to show unity power factor under NL local load. 

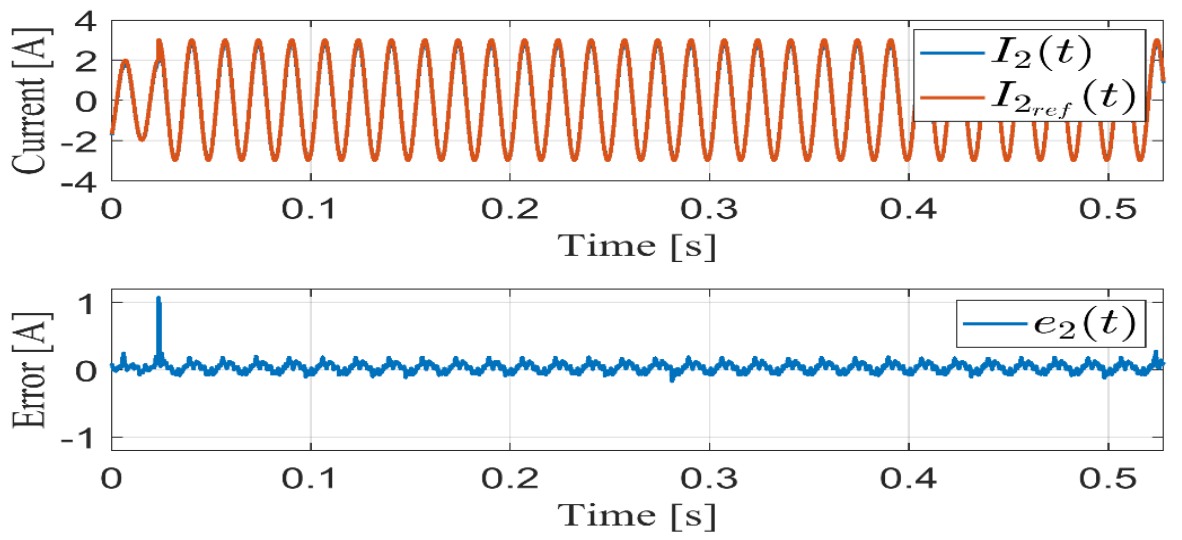

(a)
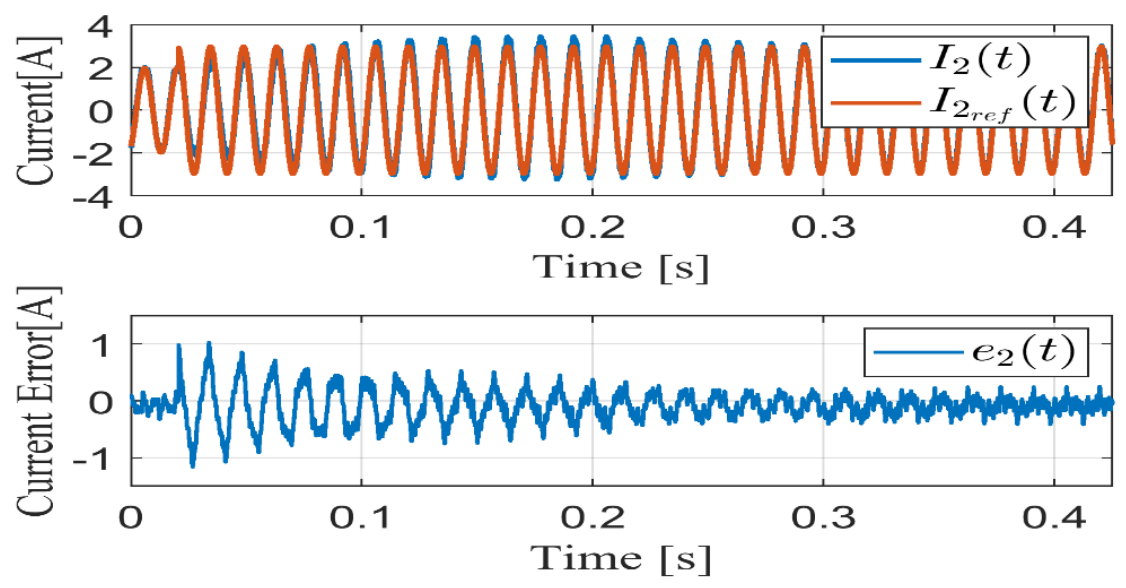

(b)

Figure 0.10 Tracking response of the grid current during reference current step change under NL load (a) Proposed Scheme, (b) Cascaded PR scheme (required gains tuning).
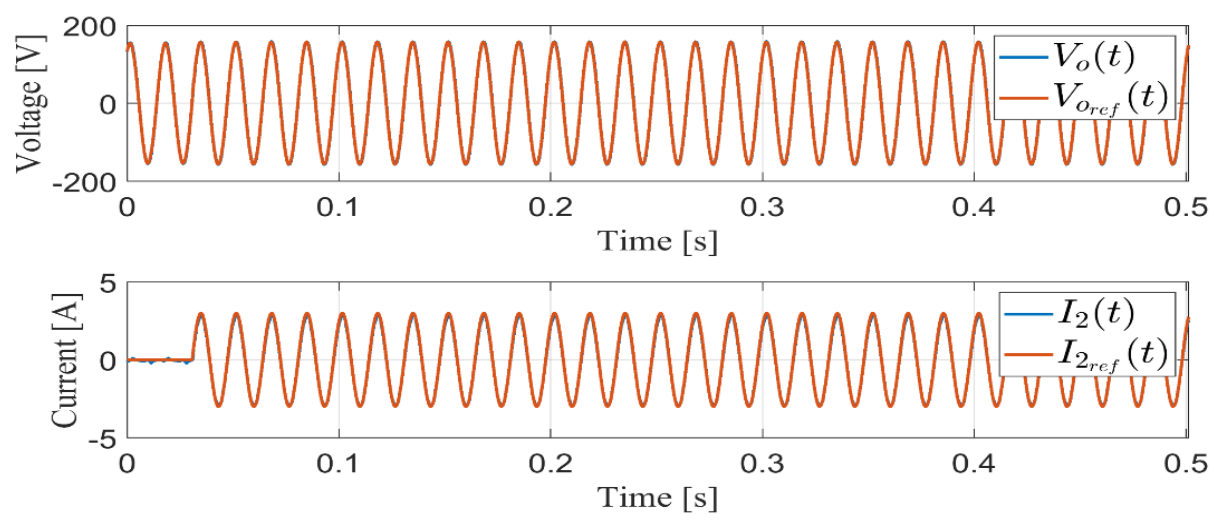

(a) 

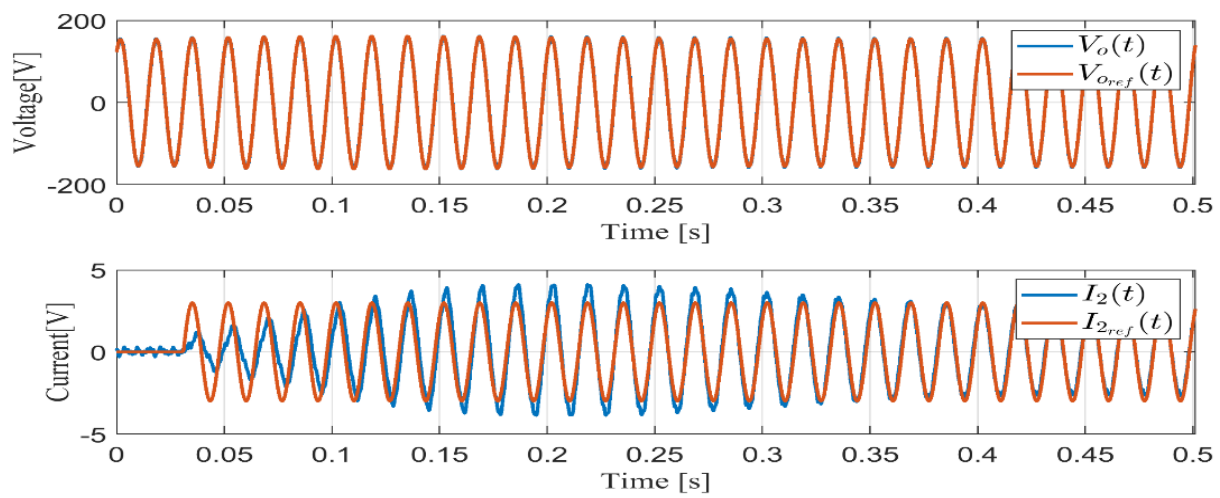

(b)

Figure 0.11 Voltage and current tracking response during transfer from SA mode to GC mode (a) Proposed Scheme, (b) Cascaded PR scheme (required gains tuning).

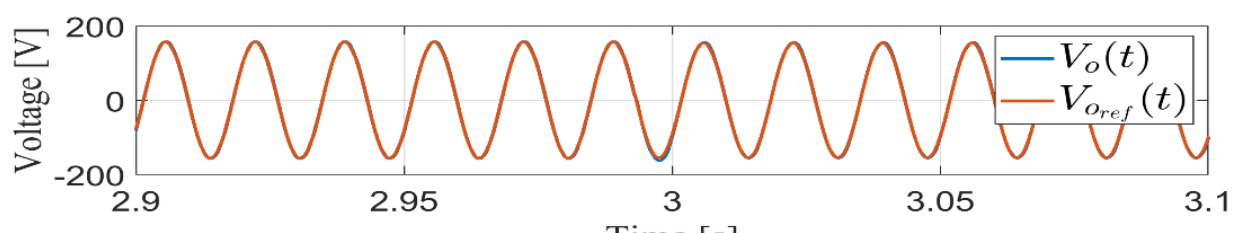

Time $[\mathrm{s}]$

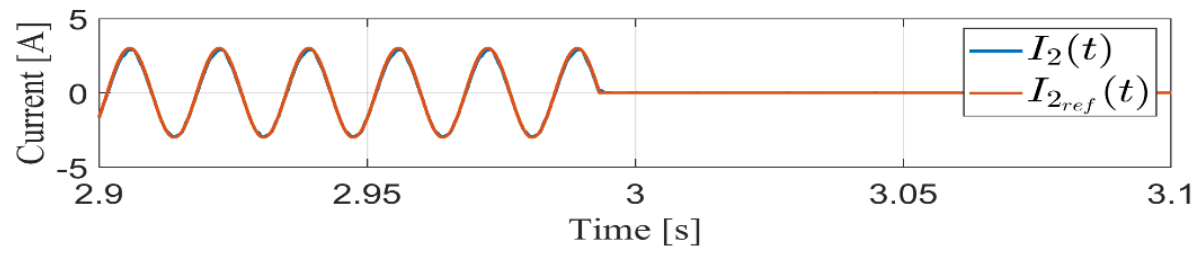

(a)
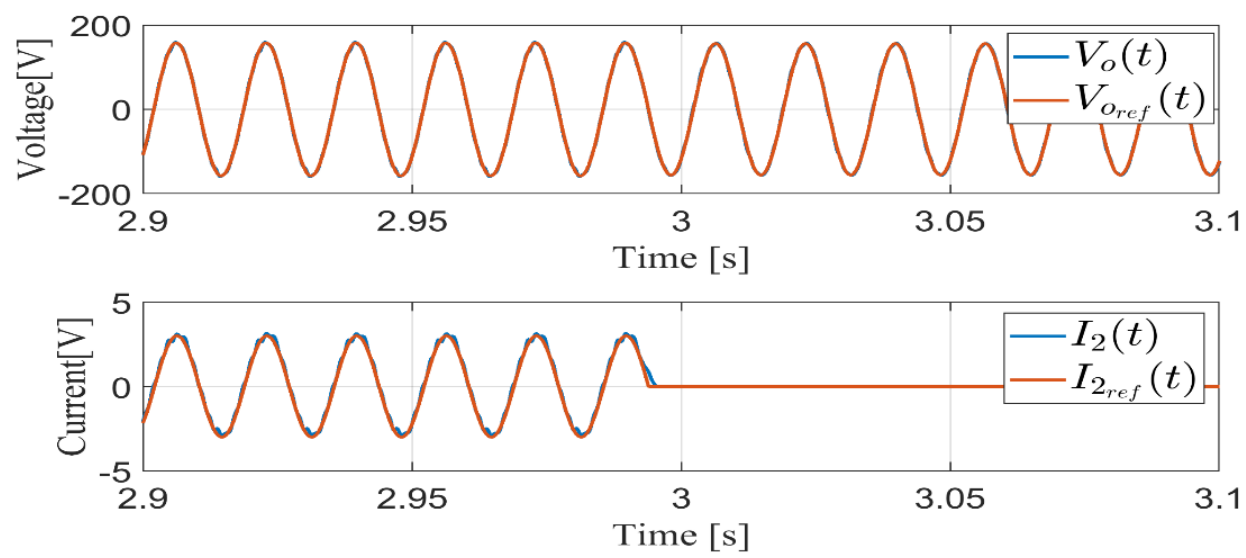

(b)

Figure 0.12 Voltage and Current tracking response during transfer from GC mode to SA mode (a) Proposed Scheme, (b) Cascaded PR scheme (required gains tuning). 


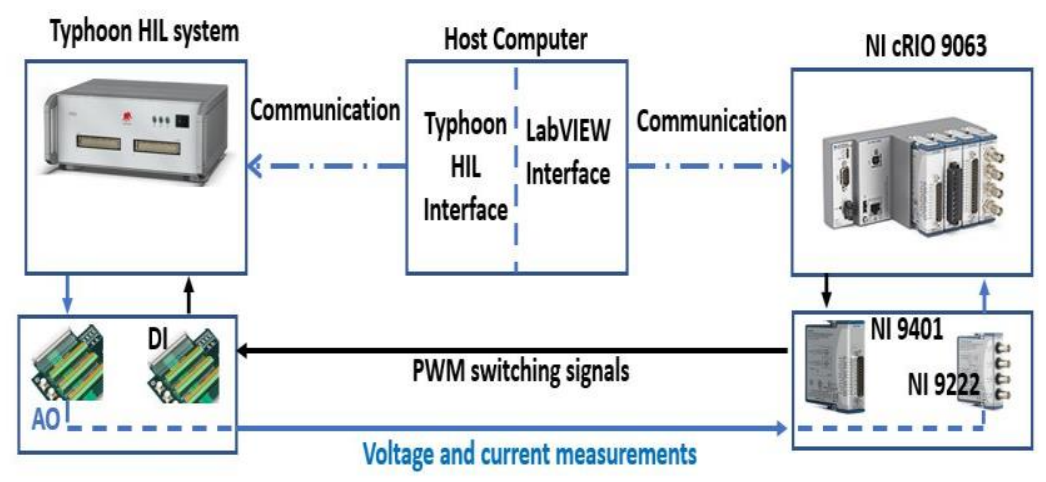

Figure 0.13 Hardware-in-the-loop (HIL) schematic diagram with LabVIEW cRIO.

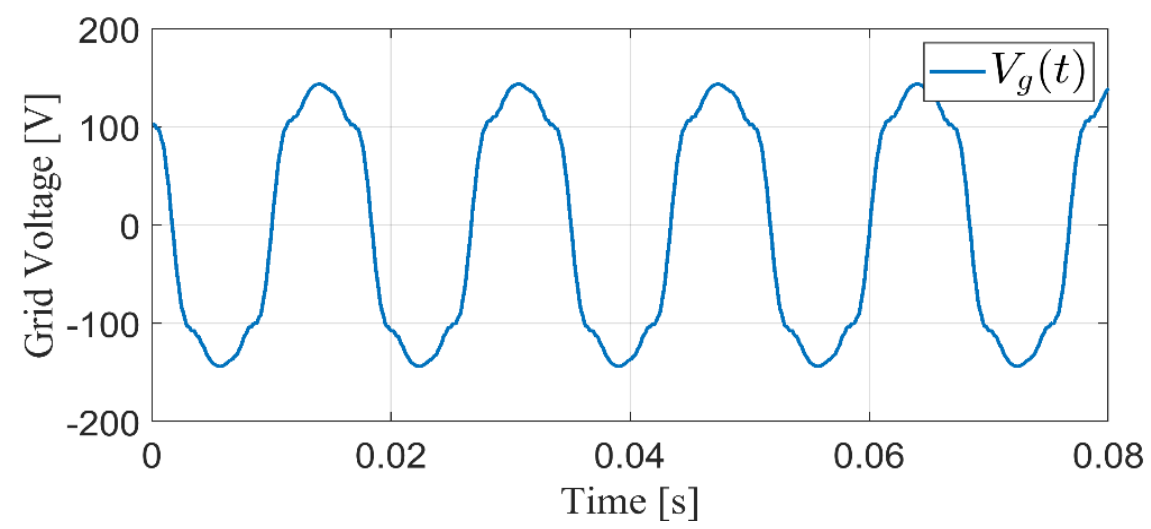

(a)

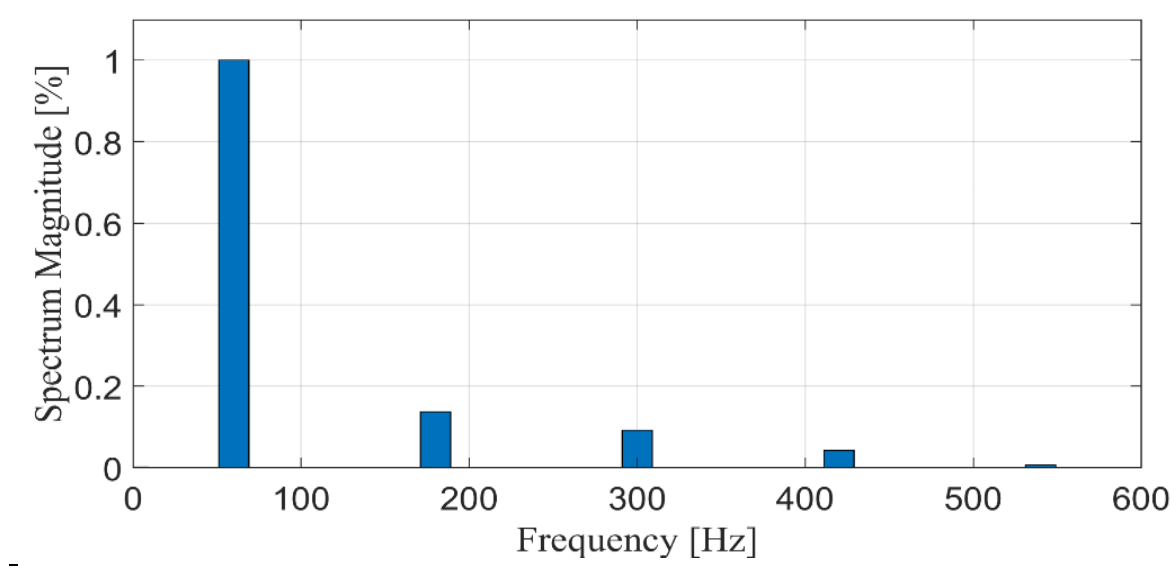

Figure 0.14 (a) Distorted grid voltage (b) spectrum analysis. 

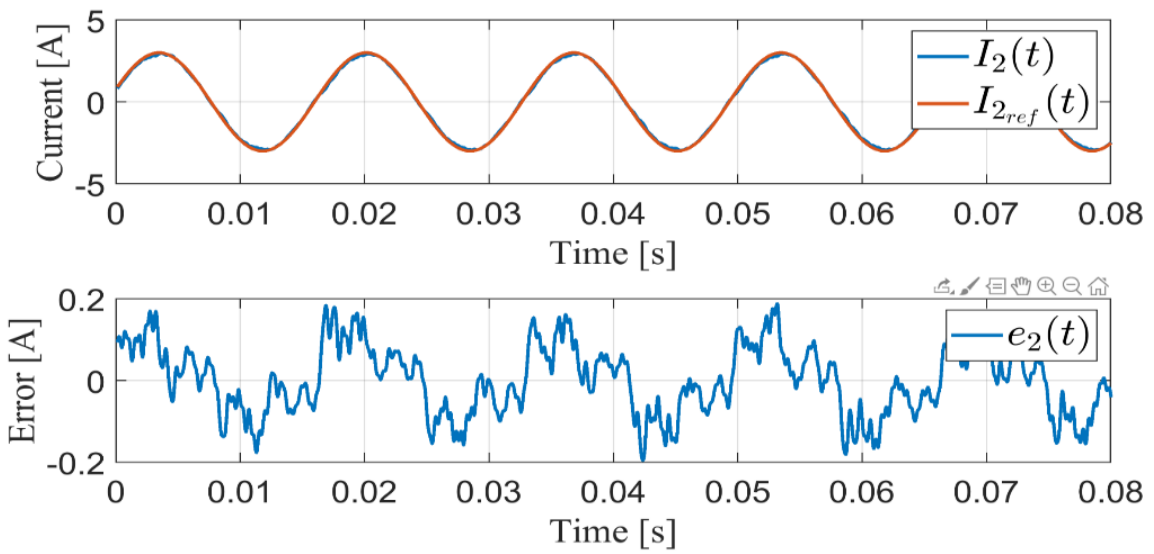

Figure 0.15 Tracking response of the grid current under distorted grid condition for NL local load.

\subsection{SUMMARY}

A filter-based nonlinear controller has been proposed in this chapter for a single-phase grid-connected inverter. The proposed scheme improves the quality of the local load voltage and the grid current simultaneously. Lyapunov stability analysis shows that the proposed controller scheme is stable, all signals in the closed loop system are bounded, and control objectives are met. An experimental testbed has been utilized to test the steadystate and the transient-state performances of the proposed scheme. The experimental results demonstrate that the proposed controller has excellent tracking performance and it shows robustness of the proposed scheme against changes in operating conditions by comparing this scheme with cascaded scheme based on standard PR controller. Also, a seamless transition between stand-alone and grid-connected modes has been achieved without changing the control structure and without resynchronization scheme. Moreover, to test the proposed scheme under distorted grid voltage, a Hardware-in-the-loop setup has been implemented by using Typhoon HIL 603. The results show that even with the highly distorted grid, the proposed control scheme working very well with low THD. 


\section{CHAPTER 6}

\section{CONCLUSION}

Different model-based nonlinear control approaches have been designed and applied to the single-phase grid-connected inverter systems to improve the quality of the local load voltage while injecting clean current to grid by using one controller. Furthermore, the proposed control schemes ensure the seamless transfer between grid-connected and standalone operation modes without adjusting the controller structure.

Since we have two control objectives, the cascaded control theory is the first candidate should be tried. The first proposed controller has an outer current loop and an inner voltage loop. Power quality of the local load voltage is the responsibility of the inner voltage controller. The role of the outer current controller is to inject clean current to the grid. In this work, a current observer has been designed to replace the inverter filter current sensor in order to reduce the impact of switching noise present in this measurement, along with system cost. In general, the main disadvantage of the cascaded control schemes is that the objective of the controller in the inner-loop should be assumed met before activating the controller in the outer-loop. To ensure that, a supervisory control is needed in the cascaded approach. As an effort to overcome this shortfall of the cascaded control approaches, the backstepping theory has been utilized to design a model-based nonlinear controller to achieve the previous two control objectives simultaneously.

The previous backstepping controller has been designed based on the inductorcapacitor dynamics with the filter inductor current as one of the system states. This current has high frequency harmonics generated from the switching devices. To avoid using this noisy current, a novel second order dynamic system equation in terms of the output voltage 
instead of the coupled inductor-capacitor dynamics has been introduced and utilized to design a controller based on the output system states. The previous control scheme requires a variable structure observer to avoid the numerical derivative of the output voltage that generated from the novel second order system dynamics. This observer increases the system complexity by adding the observer loop which in the observer objective should be met before activating the control loop. Moreover, the mathematical derivative of the grid voltage is required.

Finally, control scheme based on set of filters dynamics has been proposed to overcome the aforementioned disadvantages of the backstepping control approach with variable structure observer.

For each developed control scheme in this work, the seamless transition between stand-alone mode and grid-connected mode is ensured without changing the controller structure. Moreover, a Lyapunov stability analysis is presented which proves that the voltage and current tracking objectives are achieved by the same controller with all signals remaining bounded. Simulation and/or experimental results further validate the proposed approaches. 


\section{REFERENCES}

1. Carrasco, J.M., et al., Power-Electronic Systems for the Grid Integration of Renewable Energy Sources: A Survey. IEEE Transactions on Industrial Electronics, 2006. 53(4): p. 1002-1016.

2. Zhong, Q.-C. and T. Hornik, Control of power inverters in renewable energy and smart grid integration. 2013, Chichester, West Sussex: Wiley, A John Wiley \& Sons, Ltd., Publications. xxv, 411 pages.

3. Bao, C., et al., Step-by-Step Controller Design for LCL-Type Grid-Connected Inverter with Capacitor-Current-Feedback Active-Damping. IEEE Transactions on Power Electronics, 2014. 29(3): p. 1239-1253.

4. IEEE Standard for Interconnection and Interoperability of Distributed Energy Resources with Associated Electric Power Systems Interfaces. IEEE Std 1547-2018 (Revision of IEEE Std 1547-2003), 2018: p. 1-138.

5. Guerrero, J.M., et al., Control Strategy for Flexible Microgrid Based on Parallel Line-Interactive UPS Systems. IEEE Transactions on Industrial Electronics, 2009. 56(3): p. 726-736.

6. Pal, B., P.K. Sahu, and S. Mohapatra. A review on feedback current control techniques of grid-connected PV inverter system with LCL filter. in 2018 Technologies for Smart-City Energy Security and Power (ICSESP). 2018.

7. Ebrahimi, M., S.A. Khajehoddin, and M. Karimi-Ghartemani, Fast and Robust Single-Phase DQ Current Controller for Smart Inverter Applications. IEEE Transactions on Power Electronics, 2016. 31(5): p. 3968-3976. 
8. Chen, X., et al., Injected Grid Current Quality Improvement for a VoltageControlled Grid-Connected Inverter. IEEE Transactions on Power Electronics, 2018. 33(2): p. 1247-1258.

9. Rodriguez, P., et al., Flexible Active Power Control of Distributed Power Generation Systems During Grid Faults. IEEE Transactions on Industrial Electronics, 2007. 54(5): p. 2583-2592.

10. Nian, H. and R. Zeng, Improved control strategy for stand-alone distributed generation system under unbalanced and non-linear loads. IET Renewable Power Generation, 2011. 5(5): p. 323-331.

11. Mukherjee, S., P. Shamsi, and M. Ferdowsi. Indirect voltage control of a standalone inverter. in 2017 IEEE Applied Power Electronics Conference and Exposition (APEC). 2017.

12. Liu, C., et al. Model predictive control of single phase grid-connected inverter with LC filter. in 2017 32nd Youth Academic Annual Conference of Chinese Association of Automation (YAC). 2017.

13. Eren, S., A. Bakhshai, and P. Jain. A CLF-based nonlinear control technique for a grid-connected voltage source inverter with LCL filter used in renewable energy power conditioning systems. in 4th International Conference on Power Engineering, Energy and Electrical Drives. 2013.

14. Eren, S., A. Bakhshai, and P. Jain. A novel nonlinear current control technique for a grid-connected DC/AC inverter used in renewable energy power conditioning systems. in 2014 IEEE 36th International Telecommunications Energy Conference (INTELEC). 2014.

15. Sudhakar, B. and G.V.E.S. Kumar. Co-simulation of sliding mode control of single phase grid connected lcl filtered voltage source inverter using LabVIEW and multisim. in 2016 IEEE Region 10 Conference (TENCON). 2016. 
16. Yang, Y., K. Zhou, and F. Blaabjerg, Current Harmonics From Single-Phase GridConnected Inverters-Examination and Suppression. IEEE Journal of Emerging and Selected Topics in Power Electronics, 2016. 4(1): p. 221-233.

17. Silwal, S., et al., An Enhanced Control System for Single-Phase Inverters Interfaced With Weak and Distorted Grids. IEEE Transactions on Power Electronics, 2019. 34(12): p. 12538-12551.

18. $\mathrm{Xu}, \mathrm{J} ., \mathrm{S} . \mathrm{Xie}$, and $\mathrm{T}$. Tang, Improved control strategy with grid-voltage feedforward for LCL-filter-based inverter connected to weak grid. IET Power Electronics, 2014. 7(10): p. 2660-2671.

19. Kang, S. and K. Kim, Sliding mode harmonic compensation strategy for power quality improvement of a grid-connected inverter under distorted grid condition. IET Power Electronics, 2015. 8(8): p. 1461-1472.

20. Khalil, H.K., Nonlinear systems. 1992, New York, Maxwell Macmillan International.

21. Dehkordi, N.M., N. Sadati, and M. Hamzeh, A Robust Backstepping High-Order Sliding Mode Control Strategy for Grid-Connected DG Units With Harmonic/Interharmonic Current Compensation Capability. IEEE Transactions on Sustainable Energy, 2017. 8(2): p. 561-572.

22. Hornik, T. and Q. Zhong, A Current-Control Strategy for Voltage-Source Inverters in Microgrids Based on $H^{\wedge} \infty$ and Repetitive Control. IEEE Transactions on Power Electronics, 2011. 26(3): p. 943-952.

23. Weiss, G., et al., $H^{\wedge} \infty$ repetitive control of $D C$-AC converters in microgrids. IEEE Transactions on Power Electronics, 2004. 19(1): p. 219-230.

24. Ammeh, L., H. El Fadil, and K. Ouhaddach, Nonlinear Control of Voltage Source Inverters in Microgrids. Proceedings of Engineering and Technology-PET, 2017. 19: p. 72-77. 
25. Mohebbi, M., et al. Nonlinear control of standalone inverter with unbalanced, nonlinear load. in 2017 IEEE Power and Energy Conference at Illinois (PECI). 2017.

26. Yao, Z. and L. Xiao, Control of Single-Phase Grid-Connected Inverters With Nonlinear Loads. IEEE Transactions on Industrial Electronics, 2013. 60(4): p. 13841389.

27. Balaguer, I.J., et al., Control for Grid-Connected and Intentional Islanding Operations of Distributed Power Generation. IEEE Transactions on Industrial Electronics, 2011. 58(1): p. 147-157.

28. Kenski, D.D., T.B. Lazzarin, and R.F. Coelho. Load voltage compensation of two modes single-phase VSI. in 2017 IEEE 8th International Symposium on Power Electronics for Distributed Generation Systems (PEDG). 2017.

29. Tran, T., et al., PLL-Based Seamless Transfer Control Between Grid-Connected and Islanding Modes in Grid-Connected Inverters. IEEE Transactions on Power Electronics, 2014. 29(10): p. 5218-5228.

30. Wai, R., et al., Design of backstepping control for high-performance inverter with stand-alone and grid-connected power-supply modes. IET Power Electronics, 2013. 6(4): p. 752-762.

31. Abouloifa, A., et al., Cascade nonlinear control of shunt active power filters with average performance analysis. Control Engineering Practice, 2014. 26: p. 211-221.

32. Duan, X. and H. Zhang. Quasi-proportional resonant control of the LCL-type gridconnected inverter based on fuzzy control and self-tuning of fuzzy parameters. in 2017 29th Chinese Control And Decision Conference (CCDC). 2017.

33. Lim, K. and J. Choi. PR control based cascaded current and voltage control for seamless transfer of microgrid. in 2015 IEEE 2nd International Future Energy Electronics Conference (IFEEC). 2015. 
34. Zhong, Q.C. and T. Hornik, Cascaded Current-Voltage Control to Improve the Power Quality for a Grid-Connected Inverter With a Local Load. IEEE Transactions on Industrial Electronics, 2013. 60(4): p. 1344-1355.

35. Sahu, P.K., P. Shaw, and S. Maity. Fixed-frequency sliding mode control for power quality improvement of a grid-connected inverter. in 2015 Annual IEEE India Conference (INDICON). 2015.

36. Martins, L.T., et al., Current Control of Grid-Tied LCL-VSI With a Sliding Mode Controller in a Multiloop Approach. IEEE Transactions on Power Electronics, 2019. 34(12): p. 12356-12367.

37. He, Y., et al., Use of Boundary Control With Second-Order Switching Surface to Reduce the System Order for Deadbeat Controller in Grid-Connected Inverter. IEEE Transactions on Power Electronics, 2016. 31(3): p. 2638-2653.

38. Moath Alqatamin, M.L.M., J. Latham, Cascaded Nonlinear Control with Learning Compensation for Grid-Connected Single-Phase Inverters, in American Control Conference. 2019, IEEE: Philadelphia, PA, USA. p. 634-639.

39. Alqatamin, M. and M.L. McIntyre. Nonlinear Backstepping Controller for SinglePhase Grid-Connected Inverters. in 2019 20th Workshop on Control and Modeling for Power Electronics (COMPEL). 2019.

40. Alqatamin, M., M.L. McIntyre, and J. Latham. Backstepping Control for SinglePhase Grid-Connected Inverter Utilizing Variable Structure Observer. in 2019 American Control Conference (ACC). 2019.

41. Xian, B., et al., A discontinuous output feedback controller and velocity observer for nonlinear mechanical systems. Automatica, 2004. 40(4): p. 695-700.

42. Mohebbi, M., M.L. McIntyre, and J. Latham. A learning backstepping controller for voltage source inverter with nonlinear loads. in 2017 IEEE Power and Energy Conference at Illinois (PECI). 2017. 
43. Yang, S., et al., A Robust Control Scheme for Grid-Connected Voltage-Source Inverters. IEEE Transactions on Industrial Electronics, 2011. 58(1): p. 202-212.

44. Cortes, P., et al., Model Predictive Control of an Inverter With Output LC Filter for UPS Applications. IEEE Transactions on Industrial Electronics, 2009. 56(6): p. 1875-1883.

45. Dixon, W.E., et al., Robotic Systems, in Nonlinear Control of Engineering Systems: A Lyapunov-Based Approach, W.E. Dixon, et al., Editors. 2003, Birkhäuser Boston: Boston, MA. p. 149-222.

46. Slotine, J.-J.E. and W. Li, Applied nonlinear control. Vol. 199. 1991: Prentice hall Englewood Cliffs, NJ.

47. Yang, Y. and F. Blaabjerg. Synchronization in single-phase grid-connected photovoltaic systems under grid faults. in 2012 3rd IEEE International Symposium on Power Electronics for Distributed Generation Systems (PEDG). 2012.

48. Cortes, P., et al., Model Predictive Control of an Inverter With Output LC Filter for UPS Applications. IEEE Transactions on Industrial Electronics, 2009. 56(6): p. 1875-1883.

49. Rocabert, J., et al., Control of Power Converters in AC Microgrids. IEEE Transactions on Power Electronics, 2012. 27(11): p. 4734-4749.

50. Latham, J., M. Mohebbi, and M.L. McIntyre. Output feedback control of a single phase voltage source inverter utilizing a variable structure observer. in 2017 American Control Conference (ACC). 2017.

51. Timbus, A., et al., Evaluation of Current Controllers for Distributed Power Generation Systems. IEEE Transactions on Power Electronics, 2009. 24(3): p. 654664. 
52. Mohebbi, M., M.L. McIntyre, and J. Latham, Sensorless control of an H-bridge inverter with output inductor-capacitor filter. IET Power Electronics, 2018. 11(10): p. 1621-1627.

53. Xin, Z., et al., Grid-Current-Feedback Control for LCL-Filtered Grid Converters With Enhanced Stability. IEEE Transactions on Power Electronics, 2017. 32(4): p. 3216-3228. 


\title{
CURRICULUM VITAE
}

\author{
Moath Alqatamin \\ moadeq@gmail.com \\ Phone: 502-631-8965
}

\section{$\underline{\text { Education }}$}

University of Louisville, $K Y$, USA

Ph.D. in Electrical Engineering

Jordan University of Science and Technology, Irbid, Jordan.

Master of Science in Electrical Engineering / Control \& Power- Jan 2009.

Mu'tah Louisville, Mu'tah, Jordan.

Bachelor of Engineering/ Power - Jan 2004.

\section{Professional Experience}

Graduate Research Assistant: Electrical and Computer Engineering Department University of Louisville, Louisville, KY: (Aug 2017 - Present).

Graduate Teaching Assistant: Electrical and Computer Engineering Department University of Louisville, Louisville, KY: (Aug 2016 - July 2017).

Lecturer and Labs Coordinator: Electrical Engineering Department- King Khaled University, Saudi Arabia: (Oct 2010 -Aug 2015).

Design and Consultant Electrical Engineer: Rubia Consultant Engineering \& Partners - Jordan: (Aug 2006 - July 2010).

\section{$\underline{\text { Publications }}$}

\section{Books}

Moath Alqatamin, "Excitation Control of Synchronous Generators", LAP Lambert Academic Publishing, Germany, ISBN (978-3-659-64432-0), 02-12-2014.

\section{Peer Reviewed Journal Papers}

Moath Alqatamin, Joseph Latham, Zachary T. Smith, Brandon M. Grainger, and Michael McIntyre, " Current Control of a Three-Phase, Grid-Connected Inverter in the Presence of Unknown Grid Parameters without Phase Lock Loops" IEEE Journal of Emerging and Selected Topics in Power Electronics, DOI:10.1109/JESTPE.2020.3001153. 
Moath Alqatamin, M. L. McIntyre "Improvement the Power Quality of Single-Phase Grid-Connected Inverter by Filter-Based Control Scheme" IET Power Electronics, February 2020, DOI: 10.1049/iet-pel.2019.1212.

Moath Alqatamin "An Optimal State Feedback Controller Based Neural Networks for Synchronous Generators." International Journal in Electrical and Computer Engineering (IJECE) 3.4 (2013): 561-567. (Cited 3 times).

\section{Peer Reviewed Journal Papers (under review)}

Bhagyashri Bhagwat, Moath Alqatamin, Nicholas Hawkins, Michael McIntyre, " FilterBased Control of a Buck Converter for Uncertain Nonlinear Loads" IET Power Electronics, under review.

N. Hawkins, S. Nimon, B. Bhagwat, Moath Alqatamin, J. Latham, M. McIntyre," Nonlinear Control of CCM Power Factor Correction Circuit" IET Power Electronics, under review.

\section{Peer Reviewed Conference Papers}

Christian Pereny, Moath Alqatamin, J. Latham, B. M. Grainger, and M. L. McIntyre, " System Frequency Dynamic Response of a Novel, Self-Synchronizing Inverter in a High Renewable Penetration Grid" Accepted to be presented in IEEE ECCE-Europe, France, Sep 2020. To appear.

Moath Alqatamin, Bhagyashri Bhagwat, Nicholas Hawkins, Joseph Latham, Michael McIntyre, "Self-Synchronizing Current Control for Single-Stage Three-Phase GridConnected Photovoltaic Systems," Accepted to be presented in IEEE American Control Conference (ACC), Denver, CO, USA, July 2020. To appear.

Moath Alqatamin, Nicholas Hawkins, Michael McIntyre, "Filter-Based Controller to Improve the Power Quality of Single-Phase Grid-Connected Inverters," Accepted to be presented in IEEE American Control Conference (ACC), Denver, CO, USA, July 2020. To appear.

Nicholas Hawkins, Nicholas Jewell, Moath Alqatamin, Bhagyashri Bhagwat, Michael McIntyre, "A Nonlinear Fault Detection Scheme for PV Applications," Accepted to be presented in IEEE American Control Conference (ACC), Denver, CO, USA, July 2020. To appear.

Nicholas Hawkins, Moath Alqatamin, Bhagyashri Bhagwat, Michael McIntyre, "Nonlinear Control and Observation of a PMSG Wind Turbine Through Unknown Wind Torque Characteristics" Accepted to be presented in IEEE American Control Conference (ACC), Denver, CO, USA, July 2020. To appear.

Joseph Latham, Moath Alqatamin, Zachary T. Smith, Brandon M. Grainger, and Michael McIntyre, "Self-Synchronizing Current Control of a Three-Phase Grid- Connected Inverter in the Presence of Unknown Grid Parameters," 2020 IEEE Applied Power Electronics Conference and Exposition (APEC), New Orleans, LA, USA, 2020, pp. 793-797, doi: 
10.1109/APEC39645.2020.9123992.

Moath Alqatamin, M. L. McIntyre, J. Latham, "Backstepping Control for Grid-Connected Single-Phase Inverter Utilizing Variable Structure Observer" 2019 Annual American Control Conference (ACC), Philadelphia, PA, USA, 2019, pp. 646-651. (Cited 1 time).

Moath Alqatamin, M. L. McIntyre, J. Latham, "Cascaded Nonlinear Control with Learning Compensation for Grid-Connected Single-Phase Inverters" 2019 Annual American Control Conference (ACC), Philadelphia, PA, USA, 2019, pp. 634-639. (Cited 1 time).

Moath Alqatamin and M. L. McIntyre, "Nonlinear Backstepping Controller for SinglePhase Grid-Connected Inverters," 2019 20th Workshop on Control and Modeling for Power Electronics (COMPEL), Toronto, ON, Canada, 2019, pp. 1-6. doi: 10.1109/COMPEL.2019.8769639. (Cited 1 time).

Moath Alqatamin and M. L. McIntyre, "Nonlinear Adaptive Control for Power System with Static VAR Compensator," 2018 IEEE Electronic Power Grid (eGrid), Charleston, SC, USA, 2018, pp. 1-6.doi: 10.1109/eGRID.2018.8598695.

Moath Alqatamin, M. L. McIntyre, J. Latham, P. Rivera and N. Hawkins, "Nonlinear Adaptive Control Design for Power System with STATCOM device," 2018 Annual American Control Conference (ACC), Milwaukee, WI, 2018, pp. 3062-3067. doi: 10.23919/ACC.2018.843155. (Cited 1 time).

\section{Professional Service}

Professional Memberships

Member of the Institute of Electrical and Electronic Engineers (IEEE).

\section{Reviewer for Journals}

IEEE Transaction on Industrial Electronics

IEEE Transaction on Power Electronics

IEEE Transaction on Control System Technology

\section{Reviewer for Conferences}

IEEE Workshop on Control and Modeling of Power Electronics Systems (COMPEL).

IEEE American Controls Conference (ACC).

IEEE Conference on Decision and Control (CDC).

IEEE Energy Conversion Congress \& Expo (ECCE). 